\title{
MAISON À PÉRISTYLE ET ÉLITES URBAINES EN GAULE SOUS L'EMPIRE
}

\author{
Pascal VIPARD*
}

Mots-clés. Habitat urbain, architecture domestique, notables municipaux, romanisation.

Résumé. Les maisons à péristyle n'étaient pas simplement le type d'habitat le plus riche rencontré dans les villes romaines, mais aussi un élément du modèle de gouvernement des provinces mis en place par Rome. Leur possession devait en effet constituer une obligation pour les membres les plus éminents des élites municipales qui briguaient ou détenaient le pouvoir. Leur étude sous un angle privilégiant les parties publiques plutôt que les parties plus purement privées permet de montrer la validité pour la Gaule des modèles domestiques élaborés à Rome et la progressive intégration des Gallo-Romains aux schémas mentaux du nouveau pouvoir. Si l'exemple gaulois met en évidence de nombreux points communs avec les modèles italiques, il fait également apparaître des particularités qui sont souvent moins des provincialismes que le signe d'une évolution générale de l'usage de ces maisons dans l'Empire sous l'effet des événements historiques et des mutations des classes dirigeantes. Du fait de ses liens étroits avec le monde du pouvoir et du cadre urbain dans lequel celui-ci s'exerce, ce type d'habitat occupait une place particulière dans la ville et son existence a suivi le destin de ses élites. Apparu partout avec la naissance des villes à l'époque augustéenne, il a massivement disparu dans le courant du III ${ }^{e}$.

Key-words. Urban settlement, domestic architecture, urban elites, Romanization.

Abstract. The peristyle house was not only the most sumptuous dwelling, but also a model of government in the provinces established by Rome. Their ownership constituted an obligation for the most prominent members of the elite who solicited or held power. A study from an angle giving more importance to the public sector rather than a purely private side, enables one to to show the validity, in Gaul, of the domestic model developed in Rome and the progressive integration of Gallo-Romans in the mental scheme of the new power. If the Gallic example underlines a fair number of common points with Italic models, it also shows some particularities. These must often be considered less as provincialisms than the sign of a general evolution, under the Principate, of the use of these houses under the influence of historical events and changes inside ruling classes. Due to its strong connection with the world of power and the urban frame of its exercise, this kind of housing held a special position inside the town and its existence followed the destiny of its elites. Appearing everywhere at the birth of towns during the Augustan period, it disappeared massively in the course of the $3^{\text {rd }}$ century.

Translation: Isabelle FAUDUET and Brian GRAHAM

Schlüsselwörter. Städtische Siedlung, Wohnhausarchitektur, städtische Eliten, Romanisierung.

Zusammenfassung. Die Peristylhäuser waren nicht einfach nur der vornehmste Haustyp, den man in den römischen Städten antraf, sondern sie waren Teil des von Rom eingesetzten Regierungsmodells für die Provinzen. Für die eminentesten Mitglieder der städtischen Elite, die nach der Macht strebten oder sie innehatten, war der Besitz einer solchen Residenz wohl Pflicht. Aus der vorrangigen Untersuchung ihrer öffentlichen Bereiche vor den strikt privaten Wohntrakten geht hervor, dass die in Rom ausgearbeiteten Muster in Gallien Gültigkeit besaßen, und dass die Gallorömer sich die Denkweisen der neuen Machthaber nach und nach zu Eigen gemacht hatten. Neben zahlreichen Gemeinsamkeiten mit den italischen Mustern weist das gallische Beispiel auch Eigenheiten auf; oft handelt es sich jedoch weniger um Provinzialismen als um Merkmale, die von der Entwicklung in der Nutzung dieser Häuser im Kaiserreich unter dem Einfluss der politischen Ereignisse und der Wandlung der Führungsschichten zeugen. Durch seine enge Verbindung mit der Welt der Macht und dem städtischen Rahmen, in dem sie ausgeübt wurde, nahm dieser Wohnhaustyp einen besonderen Platz in der Stadt ein, und seine Entwicklung spiegelt das Schicksal der hier wohnenden Eliten wider. In augusteischer Zeit war er in den neugegründeten Städten im ganzen Imperium aufgetaucht und im Laufe des 3. Jh. ging seine Zahl drastisch zurück.

Übersetzung: Isa ODENHARDT-DONVEZ

\footnotetext{
* Université Nancy-II, 3 place Godefroi-de-Bouillon, BP 3317, F-54015 Nancy Cedex. Courriel : pascal.vipard@univ-nancy2.fr
} 
Le développement des fouilles urbaines des vingt-cinq dernières années a beaucoup contribué à renouveler et à enrichir la connaissance de l'habitat urbain de Gaule, et tout particulièrement celui des maisons à péristyle qui, à défaut d'être les plus représentatives de l'habitat standard, sont les plus aisément repérables.

Le premier mérite de ces découvertes a été de montrer l'universalité du type à péristyle en Gaule dont les meilleurs spécialistes doutaient encore au début des années 1990 (Desbat, 1991, p. 296-297, par exemple).

L'impression d'abondance donnée par la centaine et demie d'occurrences que l'on arrive à répertorier ${ }^{1}$ ne doit cependant pas faire illusion. Elles s'échelonnent en effet sur près de quatre siècles; leurs plans sont souvent incomplets, leur datation incertaine, les publications sommaires; la grande majorité n'est donc guère plus qu'un point sur une carte. La réflexion que l'on peut mener à partir de ce corpus documentaire assez mince est en outre compliquée par une hypothèse qui s'est fait jour il y a une dizaine d'années quand on a proposé de voir des scholae dans un certain nombre des plus grandes et plus luxueuses de ces maisons ${ }^{2}$ (Gros, 1997 ; Bouet, 2001). Si cela paraît assez convaincant pour quelques exemples, c'est sans doute loin d'être le cas pour tous parce que la plupart des arguments considérés comme déterminants peuvent en fait tout aussi bien s'appliquer à des domus. De plus, ces propositions ne tiennent pas assez compte du fait que les deux hypothèses ne s'excluent pas mutuellement. L'organisation matérielle de ces sièges de collèges étant pour le moins mal connue, rien n'interdit de supposer qu'un certain nombre d'entre eux aient pu se tenir dans la maison de l'un de leurs membres ${ }^{3}$. L'accueil de telles associations entrerait tout à fait dans le rôle public des domus aristocratiques et expliquerait la présence de certains aménagements qui paraissent à certains chercheurs ne pas devoir convenir à la demeure privée.

1. Il n'est pas possible de fournir les références détaillées ici ; on en trouvera une grande partie dans les actes du colloque d'Avignon, parus en 1996, dans Gros, 2001, p. 136-196 et dans les divers volumes de la Carte archéologique de la Gaule publiée par l'Académie des inscriptions et belles-lettres.

2. Les domus concernées sont celles d'Atys à Glanum, des Messii à Vaisonla-Romaine, des Dieux Océan à Saint-Romain-en-Gal, de la Brunette à Orange, en Narbonnaise, des Nones de Mars à Limoges, de la rue des Bouquets à Périgueux, de la place des Épars à Chartres, dans les Trois Gaules, et, de l'Insula 30 à Augst, des Insulae XVI et XXXIII à Avenches, en Germanie Supérieure.

3. Il ressort ainsi clairement d'inscriptions que pendant tout le HautEmpire, un collège aussi important que celui des frères arvales, à Rome, célébrait des cérémonies religieuses et des banquets dans la maison d'un magister, quelquefois nommément désignée par le nom de celui-ci (ILS, 5037, 5040 ou 5041, par exemple).
Ces maisons à péristyle correspondent sans doute au seul type d'habitat urbain dont la spécificité architecturale et le caractère éminemment riche s'imposent d'emblée, tout comme leur caractère universel dans l'Empire. C'est pourquoi il ne saurait être question de les étudier sous un aspect purement provincial; nous allons au contraire tenter de vérifier si la théorie générale ${ }^{4}$ peut s'appliquer à la Gaule sous l'Empire et signaler au passage certains particularismes gaulois. Nous voudrions surtout souligner l'intérêt, trop souvent négligé, de l'architecture privée pour l'histoire, sociale en l'occurrence.

L'étude des ces bâtiments est en effet l'occasion d'apporter quelques éclairages sur leurs occupants, ces élites urbaines encore mal connues dans le détail et, par la même occasion, quelques correctifs à une vision de la maison romaine encore trop souvent faussée par un modèle scolastique qui ne fait pas toujours bien ressortir le rôle que ces édifices ont joué dans la vie politique et sociale des villes provinciales.

Après avoir évoqué les principaux arguments allant dans le sens d'une liaison organique entre ces élites urbaines et les maisons à péristyle, puis avoir rappelé et montré pourquoi celles-ci ont été adoptées par celles-là, nous essaierons donc de montrer que, malgré leur apparente variabilité, ces maisons ne se caractérisent pas seulement par le péristyle qui leur sert de dénominateur commun, mais par le respect strict d'un certain nombre d'éléments-clés et par l'existence, plus aléatoire, d'autres composantes qui, pour n'être pas aussi systématiques, n'en jouent cependant pas moins un rôle dans la définition de ce type d'habitat et permettent également d'y introduire des éléments de différenciation hiérarchique. Les liens de ces maisons avec les dirigeants des cités et de nombre de leurs composantes avec le domaine public conduisent tout naturellement à évoquer les divers rapports, matériels ou spirituels, qu'elles entretiennent avec la cité où elles se dressent, jusqu'au moment de leur disparition du paysage urbain.

4. Cette théorie a été établie, pour les maisons républicaines ou du tout début de l'Empire, à partir de la documentation textuelle et archéologique italienne, de loin la plus abondante (Mackay, 1975, et surtout, Wallace-Hadrill, 1988; Coarelli, 1989; De Albentiis, 1990). On a montré qu'elle fonctionne parfaitement en Afrique (Thébert, 1985). Les connaissances sont plus sommaires et tributaires de ces études en Orient (Saliou, 1990) ou dans la péninsule Ibérique (Collectif, 1991). Une présentation générale détaillée et une bibliographie de la maison italique et dans les parties orientale et occidentale de l'Empire se trouvent dans Gros, 2001, p. 20-230. 


\section{LES DOMUS DES ÉLITES : POUR QUI ET POUR QUOI ?}

La lecture sociale que l'on donne des maisons à péristyle étudiées est issue des travaux fondateurs de A. WallaceHadrill (1988), de F. Coarelli (1989) et de leurs continuateurs, mais la base n'en reste pas moins les auteurs latins de la fin de la République, et notamment Vitruve dont il n'est pas inutile de citer ici, en préambule, un extrait du De Architectura, rédigé vers 35-25 av. J.-C., qu'il faut constamment garder à l'esprit en tenant compte des évolutions sociopolitiques intervenues depuis la rédaction de son œuvre :

«1. Quand on aura ainsi établi l'orientation des pièces on doit alors être attentif aussi au type d'architecture que requièrent, dans les constructions des particuliers, les pièces privées du maître de maison et à celui qui convient aux pièces également ouvertes aux personnes étrangères. S'agissant en effet des pièces privées, leur accès n'est réservé qu'aux seuls invités : ainsi en est-il des chambres ${ }^{5}$, des salles à manger, des bains et de toutes les autres pièces qui ont une destination de ce genre. Sont au contraire ouvertes à tous celles où chacun peut venir de plein droit, même sans invitation ${ }^{6}$ : à savoir les cours d'entrées, les cavaedia ${ }^{7}$, les péristyles et les autres pièces qui ont une fonction analogue ${ }^{8}$. En conséquence les gens de condition ordinaire n'ont aucun besoin de cours d'entrée, de tablina ou d'atriums somptueux ${ }^{9}$ puisque ce sont eux qui s'acquittent de leurs obligations sociales en se rendant chez les autres, non les autres qui se rendent ainsi chez eux. 2. [Vitruve évoque ensuite très rapidement les maisons adaptées à ceux qui vivent du produit de la campagne, aux banquiers et aux publicains, aux avocats et aux orateurs] Quant aux dignitaires, que l'exercice des plus hautes magistratures astreint à des obligations envers leurs concitoyens, il leur faut des vestibules de style royal, des atriums élevés et des péristyles ${ }^{10}$ immenses, de vastes

5. Les cubicula ont des rôles multiples, privés (chambres à coucher), mais également publics (Riggsby, 1997).

6. Il faut bien évidemment comprendre qu'il est question ici des personnes étrangères qui ont été autorisées à franchir le seuil de la maison.

7. Qui correspondent à l'atrium. Sous l'Empire, on peut raisonnablement supposer que c'est donc le vestibule élargi qui a absorbé les fonctions des deux premières parties de cet énoncé.

8. Il faut entendre par là les salles de réception (dont le nombre et l'organisation ne semblent pas avoir fait l'objet de règles très précises).

9. On remarquera qu'il n'est même pas fait mention de la possibilité qu'ils aient des péristyles.

10. Il n'est pas fait état de cet élément pour les autres catégories de personnes citées. parcs et promenades d'une beauté majestueuse; il leur faut aussi des bibliothèques, des galeries de tableaux et des basiliques ${ }^{11}$ dont la magnificence de réalisation ne le cède en rien à celle des bâtiments publics : c'est fréquemment en effet dans leurs demeures qu'interviennent les délibérations publiques ainsi que les jugements et les arbitrages privés. 3. Si donc les constructions sont ainsi aménagées, suivant ces principes, en fonction de chaque catégorie sociale, comme il a été écrit au premier livre à propos de la beauté fonctionnelle, il n'y aura rien à reprendre ; tout en effet y sera opportunément et parfaitement ordonné [...]. » (Vitruve, De Architectura, VI, 5 : "statut social et types d'habitation », trad. Callebat, 2004, p. 20-21).

\section{LES ÉLITES, PROPRIÉTAIRES DES DOMUS À PÉRISTYLE}

Les textes sont pour le moins discrets sur les maisons de Gaule et, plus encore, sur leurs propriétaires. Presque toutes les maisons à péristyle gauloises sont donc anonymes. Les rares exceptions sont généralement hypothétiques, comme c'est le cas à Vaison-la-Romaine (Vaucluse) ${ }^{12}$, ou problématiques, comme dans le cas de la domus de la rue des Bouquets à Périgueux (Dordogne) ${ }^{13}$. Quand un nom peut être retenu, il ne renseigne finalement qu'assez peu sur le statut et les fonctions réels du propriétaire : ainsi le Balbius Iassus de la maison éponyme d'Autun (Saône-etLoire) $(A E, 1916,123)$ ou, peut-être, les porteurs de tria nomina se cachant derrière les T. VA[lerius] SAT[urninus?] et L. TIN[cius] ?. PR[...] inscrits sur des tuyaux en plomb de certains états antérieurs à la Maison des Dieux Océan à Saint-Romain-en-Gal (Rhône) (Desbat et al., 1994, p. 189).

11. À partir d'Auguste, le rôle judiciaire va disparaître du cadre domestique; on n'en trouve donc pas dans les maisons du Haut-Empire (Gros, 2004).

12. Une dédicace retrouvée en haut de la rue des Colonnes pourrait laisser supposer que la Maison au Dauphin a appartenu à la famille d'Antistia Pia Quintilla, fille de Quintus, flaminique de Saint-Paul-TroisChâteaux (Goudineau, de Kisch, 1991, p. 45-46 et p. 56). Une dédicace mentionnant une certaine Messia Alpina en remploi dans un montant de porte est à l'origine de la dénomination de la Maison dite des Messii (ibid., p. 56).

13. Elle appartenait très probablement à la puissante gens pétrucore des Pompeii dont on soupçonne un des membres d'avoir donné le terrain adjacent pour la construction de l'enceinte et des basilicae du temple circulaire adjacent (ILA Petrucores, 12-14 ; Barrière, Sarradet, 1996 , p. 156-160 et graffitis mentionnant cette famille dans Barbet et al., 2004, p. 159 et p. 161) ; la nature domestique du bâtiment a cependant été mise en doute au profit d'une schola (Bouet, 2001, p. 241-260 ; Barbet et al., 2003, p. 86). 


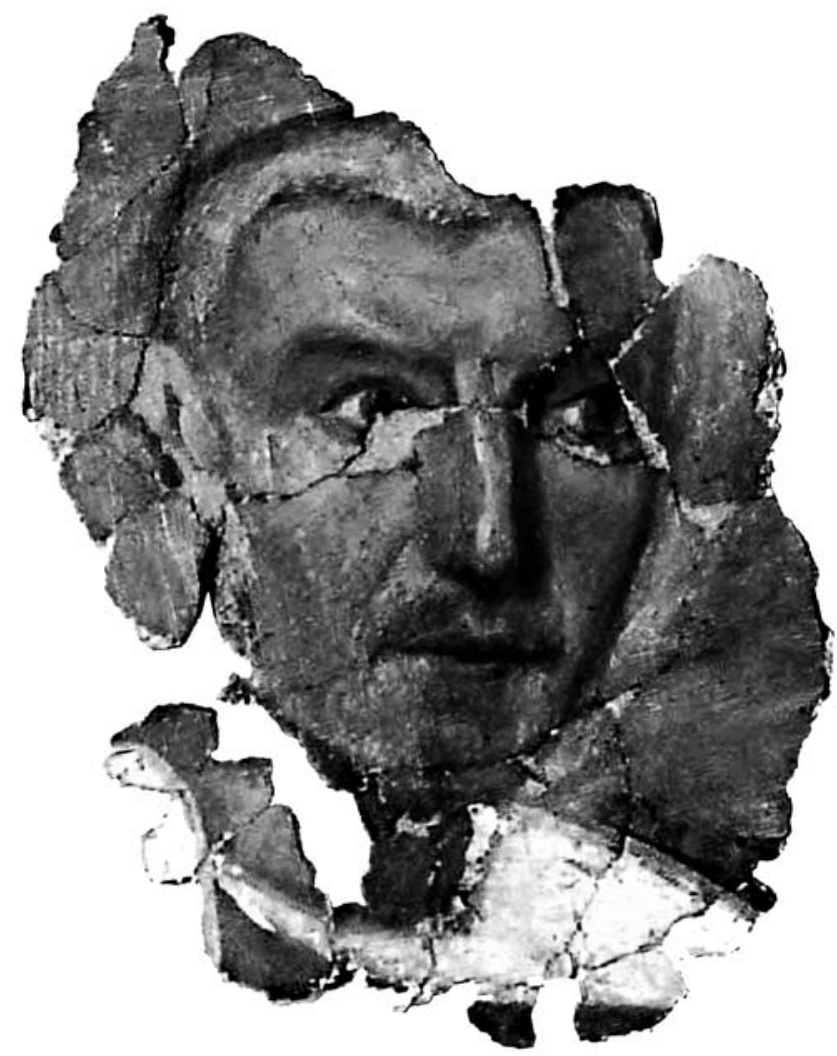

Fig. 1 - Visage réaliste, grandeur nature, d'un homme (le dominus ?) dans la Maison A du site de la rue de la Harpe à Évreux (Eure), fin du II ${ }^{e}$ s.-1re moitié du III ${ }^{e}$ s. (d'après Collectif, 1994, p. 175).

Il n'y a guère plus à tirer des rares portraits supposés être ceux des propriétaires (fig. 1) (Vipard, 2004).

En Gaule, le lien entre les maisons à péristyle et les membres les plus puissants des aristocraties municipales 14 (hypothèse la plus communément et implicitement admise) relèverait donc quasiment du postulat si les témoignages allant dans ce sens dans d'autres provinces n'étaient tous concordants et si l'interprétation que l'on peut faire des vestiges archéologiques conservés ne coïncidait pas si bien avec les besoins de cette classe sociale.

Ces primores civitatis ${ }^{15}$ correspondent à l'élite de la population urbaine considérée d'un point de vue social plus que

14. On laissera de côté le problème des quelques maisons à péristyle qui ont pu être des sièges de collèges (Gros, 1997) ou avoir appartenu à une personne morale ou à l'empereur. Dans la plupart des cas, elles semblent avoir d'abord appartenu à des individus avant d'être transformées, confisquées ou léguées et, à ce titre, ne diffèrent donc pas fondamentalement des autres à un moment de leur histoire.

15. Expression employée à l'époque sévérienne par le jurisconsulte Modestinus pour désigner les décideurs de la cité (Digeste, XXXIII, $2,11)$. strictement juridique, et pas seulement aux seuls membres de sénats locaux et aux magistrats ${ }^{16}$, mais également à ses autres habitants les plus riches (chevaliers, affranchis, négociants et incolae en voie d'intégration à l'ordo), en fait, à l'ensemble des gens riches ${ }^{17}$ susceptibles de jouer un rôle actif dans une cité (gestion des magistratures et évergésies).

Pour en revenir aux maisons à péristyle, plusieurs arguments, de valeurs variables mais très liés entre eux, vont dans le sens de leur association à ces notables, et plus précisément même aux seuls membres les plus riches: parmi eux, d'abord, et surtout, le fait qu'elles présentent des caractéristiques réservées aux détenteurs du pouvoir, mais également, renforçant le précédent, leur coût et le fait qu'on ne les trouve pour ainsi dire que dans les chefs-lieux de cité, qu'elles y sont rares et que leur nombre est de loin inférieur au nombre des membres de l'ordo.

L'argument sans doute le plus fort pour rattacher ces maisons aux primores civitatis réside dans leur forme et dans la présence systématique d'éléments qui, même si l'on considère, comme P. Gros, une adoption de formes sans en comprendre le sens au début, n'en répondent pas moins à un usage social précis, clairement formulé par Vitruve dans les sixième et septième livres du De Architectura. Au moment du développement de l'urbanisme en Narbonnaise et peu de temps avant la mise en place de celui des Trois Gaules, la théorie architecturale (notamment domestique) est en effet soigneusement mise au service de la construction de la nouvelle société voulue par Auguste et il serait étonnant que les maisons qui commencent précisément à éclore un peu partout en Gaule à cette époque n'en suivent pas les préceptes.

Un deuxième argument repose sur le prix probable de ces maisons. Un faisceau d'indices permet de s'en faire une idée très approximative et de supposer qu'une domus à péristyle de bonne taille devait largement dépasser les 100000 sesterces et, plus couramment, atteindre plusieurs centaines de milliers de sesterces, avec de grandes variations d'une cité ou d'un propriétaire à l'autre. Un essai de quantification des matériaux et de la main-d'œuvre nécessaires à l'édification d'une domus de ce type (Vipard, 1996, p. 155-165 ; Vipard, Rioult, 1999) permet également de

16. Ces derniers n'appartenaient d'ailleurs pas nécessairement à l'ordo decurionum. Sur les conditions d'adlectio dans ce dernier, en Gaule, voir la mise au point de A. Pelletier, 2000.

17. Sur les sources (discrètes) de la fortune foncière des notables de Gaule, voir Drinkwater, 1979 ; sur d'éventuelles sources non foncières, voir Picard, 1987-1988; en général, voir la série d'articles parus dans Ktèma, $\mathrm{n}^{\text {os }} 2$ et 3 , en 1977 et 1978 sur le thème des « Sources et formes de la richesse dans le monde romain » et Leveau dir., 1988. 


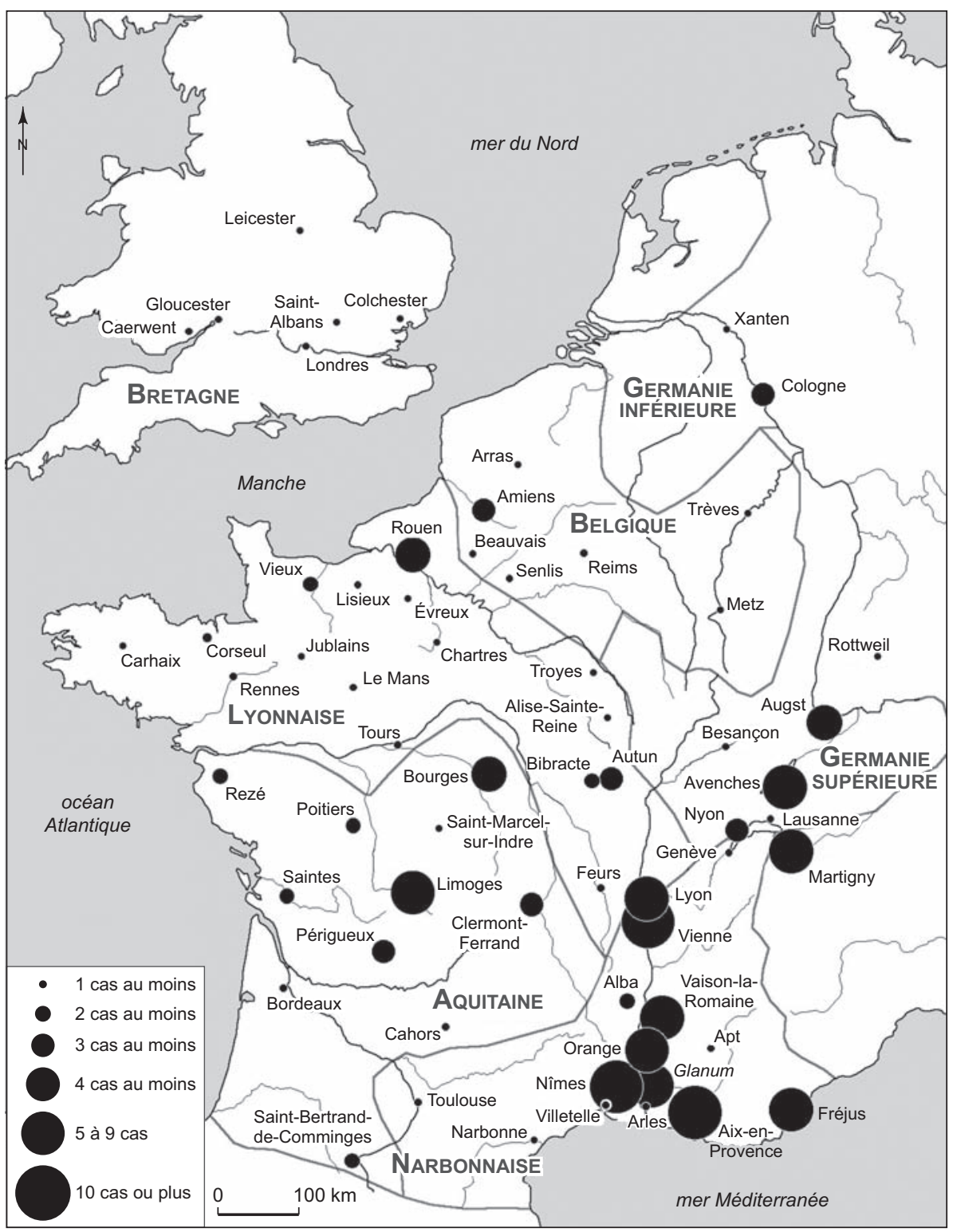

Fig. 2 - Carte de localisation des principales maisons à péristyle connues en Gaule, en Germanie et en Bretagne (DAO : P. Vipard, Université Nancy-II). pressentir que les propriétaires dotés d'une solide fortune foncière ont $\mathrm{pu}$ construire à moindres frais, sans pour autant lésiner sur la qualité, en utilisant au maximum les potentiels géologiques et humains de leurs domaines.

En fait, le coût réel de la résidence, sans être négligeable, est sans doute peu élevé par rapport à la fortune de certains propriétaires et négligeable par rapport au coût global de la construction. Une grande partie de ce dernier tient dans ce qu'il a fallu dépenser, en amont, pour se procurer un terrain bien situé en ville et acheter les bâtiments qu'il a généralement fallu raser, mais surtout, en aval, dans les frais, apparemment infiniment plus considérables, de déco- ration et d'ameublement, de maintien du train de vie (fonctionnement, entretien, clientèle, etc.), et, au-delà encore, dans les dépenses à assurer pour avoir ainsi fait montre de sa puissance. La maison à péristyle semble bien, en effet, avoir été la forme la plus évidente de manifestation de la dignitas requise pour accéder au pouvoir et de la possession d'une fortune permettant l'accès aux plus hautes fonctions avec toutes les dépenses que cela impliquait (évergésies, frais de représentation, etc.)

De ce fait, les gens, même riches mais qui n'avaient pas les moyens de participer à la vie sociale sous peine d'être vite ruinés, n'avaient sans doute pas intérêt à afficher trop 
ouvertement leur richesse en habitant une telle maison. À cet égard, un épisode de l'Âne d'or d'Apulée ${ }^{18}$ illustre remarquablement le fait qu'au $\mathrm{II}^{\mathrm{e}} \mathrm{s}$. apr. J.-C., la possession d'une telle demeure est bien une forme consciente d'ostentation constituant une pratique politique lourde de conséquences : le riche banquier Chryseros, d'Hypata en Thessalie, "par crainte des charges publiques et des obligations qu'elle comporte, [...] mettait une grande ingéniosité à dissimuler une grande fortune "; l'un des points essentiels de cette dissimulation de son état étant précisément de se contenter d'une «petite maison » destinée à ne pas attirer l'attention.

Il paraît assez difficile de concevoir qu'en ville, où l'on sait que les membres de l'ordo présentaient des niveaux de richesse extrêmement variés et ne possédaient donc pas tous une domus à péristyle, certaines personnes qui n'auraient pas exercé de rôle civique aient pu se faire construire ou acheter de telles maisons sans posséder la dignitas requise.

Un troisième argument en faveur d'un rattachement des maisons à péristyle aux notables est le fait que leurs occurrences sont très majoritairement localisées dans les chefslieux de cité, sièges du pouvoir (fig. 2). Et les quelques exceptions, des maisons plus modestes d'ailleurs : Ratiatum/Rezé («seconde ville des Pictons » d'après Ptolémée, Géographie, II, 7, 5), Argentomagus/Saint-Marcel-sur-Indre (chez les Bituriges Cubi), Lousonna/Lausanne (chez les Helvètes) et Genève (chez les Allobroges), concernent de très importantes agglomérations secondaires, sans doute des sièges de pagi où pouvaient donc s'exercer des fonctions politiques secondaires calquées sur celles des chefs-lieux.

Cette localisation pourrait être due, tout autant qu'à l'exercice de l'ostentation (inconcevable ailleurs que devant un public), à l'obligation faite aux décurions de résider dans un chef-lieu de cité. Cette obligation n'est juridiquement bien attestée que dans les municipes (voir la loi d'Urso, en Bétique, notamment), mais on peut supposer un alignement sur ces standards dans les cités pérégrines. Ce que l'on ne sait pas, en revanche, pour l'époque impériale, c'est si cette contrainte institutionnelle s'accompagnait de certaines exigences concernant la nature, la taille ou le prix de la résidence. Le dernier point est toutefois probable puisque la possession d'une maison constituait une garantie financière (assurant, comme du reste le cens exigé, la solvabilité individuelle du magistrat ou collective de l'ordo, pour la collecte de l'impôt notamment). Pour le reste, seuls la richesse et le degré d'intégration de chacun devaient présider à la réalisation des domus.

18. L'Âne d'or ou Les Métamorphoses, IV, 9, trad. P. Grimal, Paris, Gallimard, 1975, p. 97.
Plus corollaire du prix que de l'appartenance même à la classe des notables, la rareté des maisons à péristyle constitue un dernier argument, qui restreint d'ailleurs leur possession aux plus riches d'entre eux. Le faible nombre de maisons à péristyle mises au jour dans les villes gauloises par rapport au reste de l'habitat est une constante et, même en tenant compte du fait qu'une grande partie de leur sous-sol reste inconnue (défaut compensé par le fait que ce type d'habitat attire aussi davantage l'attention des observateurs), les cas de villes comptant plus d'une demi-douzaine de maisons contemporaines sont très rares, même en Narbonnaise (fig. 2). En Germanie Inférieure, conquise au cours du $\mathrm{I}^{\mathrm{er}} \mathrm{s}$. et très faiblement urbanisée, seule Cologne, chef-lieu de colonie, en a livré plus d'une. En Bretagne, sans que l'on puisse faire intervenir une éventuelle différence de statut urbain ${ }^{19}$, la maison à péristyle semble avoir également été d'une insigne rareté face à la maison à corridor ou de type villa rurale (d'un niveau social comparable ?) ; donc, même en ne tenant compte que de la taille, on arrive respectivement à une douzaine et à une quinzaine de grandes demeures (dont aucune à péristyle) pour les 80 ha intra muros de Viroconium/Wroxeter et pour les 40 ha intra muros de Calleva Atrebatum/Silchester, et à seize grandes domus (dont une seule à péristyle) pour les 15 ha intra muros de Venta Silurum/Caerwent (Wacher, 1981, p. 372 et fig. 60 ; p. 388 et fig. 82).

Bien qu'ancienneté de la romanisation et importance du statut de la cité soient des facteurs favorables, il ressort donc bien que le modèle est présent presque partout dans des proportions très diverses, mais jamais en très grand nombre, quel que soit le statut juridique du chef-lieu concerné.

Ces quelques éléments quantitatifs, tout entachés d'imperfection qu'ils soient, font donc clairement apparaître qu'il ne saurait y avoir eu autant de maisons à péristyle (ou seulement même très riches) que de décurions ${ }^{20}$. Cela rejoint ce que l'on sait par ailleurs sur les disparités de fortune des membres de l'ordre décurional où beaucoup ne devaient pas posséder un revenu familial annuel de plus de 1500 ou 2000 sesterces et sur le fait que, dans les cités moyennes, les familles détenant au moins 100000 sesterces constituaient une élite (Jacques, 1984, p. 532 et p. 535).

19. On trouve en effet des maisons à péristyle dans des colonies (Gloucester, Colchester) aussi bien que dans des cités pérégrines (Leicester, Caerwent, Saint-Albans).

20. En prenant pour base le chiffre de 100 membres établi pour l'Italie et apparemment indépendant de celui de la population (DuncanJones, 1965, p. 217 et p. 283), chiffre généralement admis pour les autres cités d'Occident, même si des cas de 50 à 80 membres ont pu exister (Jacques, Scheid, 1990, p. 258). 


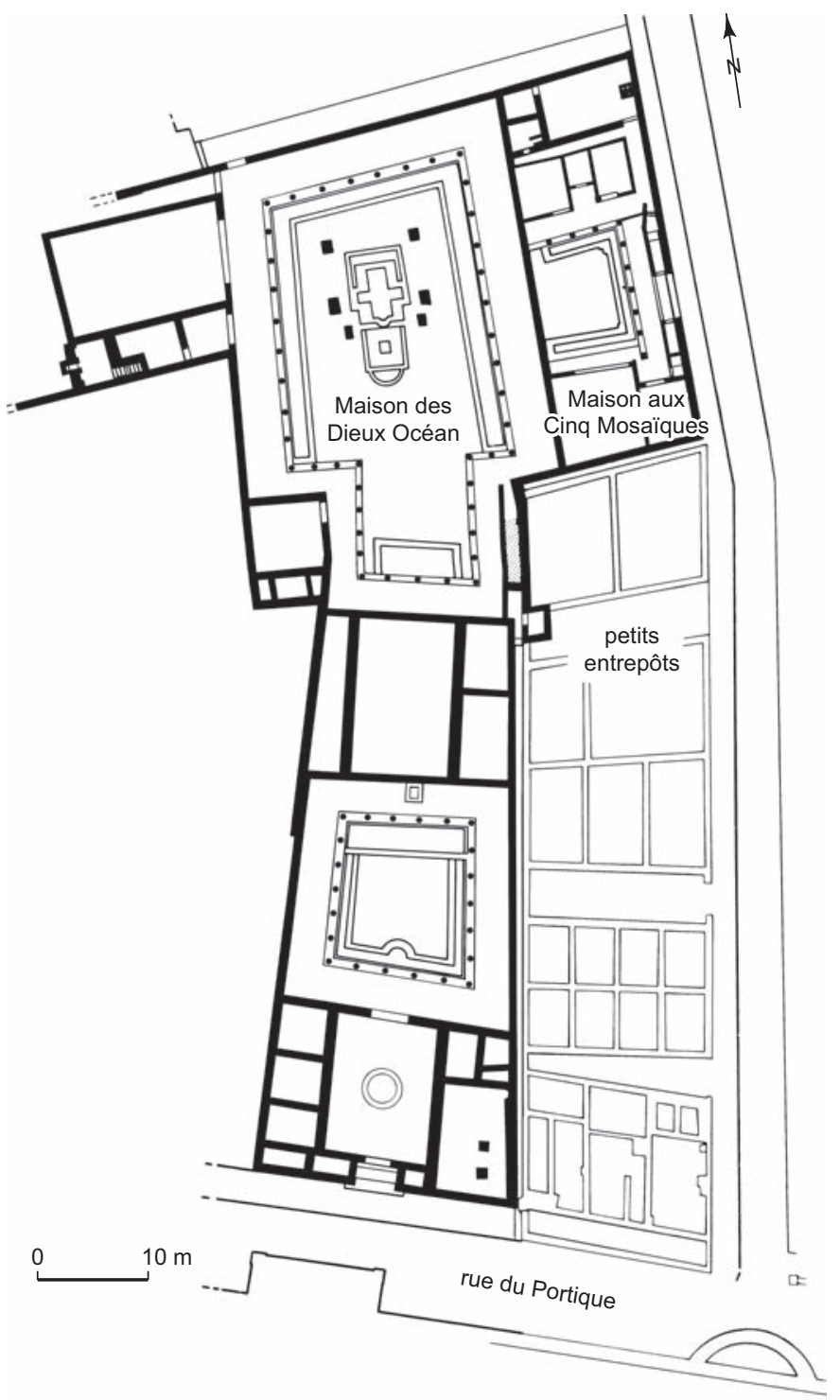

Fig. 3 -Deux maisons à péristyle : la Maison des Dieux Océan et la Maison aux Cinq Mosä̈ques à Saint-Romain-en-Gal (Rhône) (d'après Collectif, 1996b, p. 383 et p. 387).

Dans ces conditions, on imagine facilement qu'une telle maison n'était pas à la portée de tous, mais seulement des plus puissants d'entre eux ${ }^{21}$.

Par conséquent, l'existence, mais surtout le nombre de maisons à péristyle ont de forte chance d'avoir un excellent rapport avec la puissance économique d'une cité, le degré de romanisation de ses habitants et leur volonté de montrer leur intégration, mais dépendent sans doute également des

21. Cette rareté est encore accrue par le fait que certains propriétaires de maisons à péristyle pouvaient en posséder plusieurs dans une même cité, voire dans des cités différentes (Digeste, XXX, 41, 5). différences de richesse et de prestige social des membres de l'aristocratie municipale ainsi que du statut économique et politique de chaque centre urbain. La masse des décurions modestes et des autres notables devait habiter d'autres types de maisons en rapport avec leur rang.

Toutefois, il paraît raisonnable de supposer que la maison à péristyle, de par les valeurs de romanité qu'elle véhiculait, a dû constituer un but pour beaucoup. Ainsi, des maisons à péristyle de dimensions réduites nous livreraient peut-être des exemples d'habitat de décurions modestes, notamment celles dotées de l'eau courante (fig. 3 et tabl. III).

En choisissant une telle maison, modèle dominant à Rome même, son propriétaire exprimait clairement son appartenance à la gestion de l'Empire et asseyait de façon prestigieuse sa position auprès de ses concitoyens (et inférieurs) dans sa cité (Gros, 2001, p. 136). Concurremment à sa valeur idéologique, la maison permettait également de mettre concrètement en ouvre les mécanismes du pouvoir auxquels les notables aspiraient à participer. Au début de l'Empire, est en effet toujours bien présente dans les esprits l'étroite liaison existant à l'époque tardo-républicaine entre la richesse, la possession d'une demeure et le train de vie fastueux comme base essentielle à l'acquisition de la dignitas et du pouvoir (par le biais des liens de dépendance) ${ }^{22}$.

\section{LE RÔLE SOCIAL DE LA MAISON : UN INSTRUMENT DE POUVOIR}

Pour les plus riches citoyens, la maison urbaine ne répondait pas, en effet, au simple besoin de se loger, mais sans doute bien plus encore à la satisfaction de l'otium et du negotium (le traitement des affaires tant privées que publiques), et notamment d'un certain nombre de contraintes sociales découlant de ce dernier.

La première semble avoir été l'obligation de résider en ville faite aux membres de l'ordo, sans que l'on sache toutefois si elle s'accompagnait d'obligations (pas nécessairement écrites) d'importance ou de valeur minimales ${ }^{23}$. Pour les plus hauts magistrats, il se pourrait même que la possession

22. Voir, à la fin du I ${ }^{\mathrm{er}}$ s. apr. J.-C., Tacite, Annales, III, 55, 2 : « [...] quisque opibus domo paratu speciosus per nomen et clientelas inlustrior habebatur [...]» (trad. : «[...] quiconque brillait par ses richesses, par sa maison et par sa magnificence était tenu pour plus illustre encore par son renom et par ses clientèles [...]»).

23. Seule la loi municipale tardo-républicaine de Tarente impose aux décurions de cette ville d'y posséder une maison d'au moins 1500 tuiles (ILS, $6086=$ FIRA, I, 18, ligne 28), ce qui ne paraît pas correspondre à une dimension minimale très élevée. Elle établit cependant clairement l'existence d'un rapport légal entre dignitas et superficie de la domus. 
d'une maison ait constitué tout ou partie des praedia servant de caution. On ne possède pas de témoignages implicites pour la Gaule, mais on peut supposer un alignement sur des standards universels tels que les décrivent diverses lois municipales espagnoles (dont on peut seulement supposer l'application pour les cités pérégrines). Dans le dernier tiers du I ${ }^{\mathrm{er}}$ s. apr. J.-C., la rubrique LX de la lex Irnitana, par exemple, évoque en effet les propriétés servant de garanties aux magistrats ${ }^{24}$ (González, 1986).

Une deuxième contrainte concernait la dignitas exigée pour tout notable, dont la maison constituait l'une des manifestations essentielles et sans doute la plus immédiatement perceptible par les contemporains.

À l'époque républicaine, si la maison était un élément capital de la dignitas, elle n'en était pas l'instrument d'acquisition, mais seulement le résultat ; l'acquisition de la dignitas par la possession d'une demeure luxueuse était alors moralement condamnée, ce qui prouve toutefois que la pratique était déjà inscrite dans les faits. Dignitas et luxuria sont en effet intimement liées et l'ostentatio que permet la seconde est légitimée par la première ${ }^{25}$. Vitruve ne porte d'ailleurs pas de jugement moral sur l'ostentatio et la luxuria (puisqu'il est censé s'adresser à des gens dont la dignitas est acquise), mais insiste surtout sur leur nécessité fonctionnelle.

Rien ne permet de supposer, à travers ce que l'on sait de la politique impériale ou de la composition des élites urbaines, que cet opprobre moral, non de la luxuria en ellemême mais comme instrument de promotion sociale, n'a pas perduré en Gaule pendant toute la durée d'existence de ce type de maison. Au début de l'Empire, cela ressort clairement des premières maisons à péristyle connues qui semblent continuer à privilégier la dimension des pièces ou des bâtiments plutôt que le luxe ; celui-ci, pour majestueux qu'il puisse être, n'en semble pas moins très respectueux des modes alors en vigueur (III ${ }^{\mathrm{e}}$ style essentiellement) et, finalement, relativement discret, en total respect avec la politique de simplicité des mœurs des premiers empereurs. Par la suite, il faut se garder d'interpréter trop rapide-

24. Parmi les dispositions de la lex Irnitana, on doit retenir la désignation d'office de candidats en cas d'absence de volontaires, ce qui montre le risque qu'il pouvait y avoir à attirer l'attention sur soi, par exemple en affichant sa richesse dans une maison.

25. Cette condamnation morale est un poncif de la littérature de cette époque dont on trouve l'expression la plus achevée dans un passage du De Officiis (I, 39, 138-140) où Cicéron résume clairement son point de vue selon lequel une magnifique demeure ne saurait tenir lieu de dignitas pour ceux qui aspirent à celle-ci et aux honneurs à travers l'exhibition de leur luxe, ce dernier n'étant toléré que chez les principes civitatis dont la position politique n'est pas douteuse et la dignitas acquise. ment, comme une diminution ou une disparition de cette condamnation, l'accroissement régulier et sensible du luxe (au détriment de la taille) qui atteindra son point culminant dans la seconde moitié du $\mathrm{II}^{\mathrm{e}}$ s. et au début du $\mathrm{III}^{\mathrm{e}}$ s., parallèlement à l'étoffement et au renouvellement des classes dirigeantes des cités à partir de la seconde moitié du $\mathrm{I}^{\mathrm{er}} \mathrm{s}$. (Drinkwater, 1978). Rien ne prouve en effet que les homines novi n'aient pas acquis une dignitas avant de posséder éventuellement une maison à péristyle ${ }^{26}$.

Une troisième contrainte importante pour un notable était la nécessité de s'acquitter dignement des fonctions sociales liées à son état. Encore une fois, la maison était un instrument privilégié pour cette tâche, comme cela se trouve résumé, au moment où apparaissent les premières maisons à péristyle de Narbonnaise, dans le passage de Vitruve déjà évoqué (De Architectura, VI, 5, 1). Plus encore que dans le traitement dans le cadre domestique d'affaires tant privées que publiques au sujet desquelles on ne sait rien de très précis, la maison à péristyle jouait un rôle capital dans la pratique du patronage, introduite chez les aristocrates municipaux et qui justifiait amplement l'adoption par ceux-ci d'un certain nombre de formes architecturales domestiques adaptées à l'exercice de cette forme de pouvoir. Elle permettait notamment la réception matinale, en masse pour la plupart, en petit comité pour d'autres, voire en audience privée pour certains, suivant leur position dans la hiérarchie des clients.

Il n'y a aucune raison de supposer que les organes de réception de la maison romaine n'aient pas rempli leur fonction originelle durant tout le Haut-Empire puisque, jusqu'au $\mathrm{III}^{\mathrm{e}} \mathrm{s}$. encore, des données littéraires ou épigraphiques attestent la pratique du clientélisme dans de nombreuses cités. La carrière d'hommes politiques de grande envergure, dont les plus célèbres exemples gaulois sont sans doute les flamines de la province de Narbonnaise ou les prêtres fédéraux de l'Autel des Trois Gaules à Lyon, ne s'explique d'ailleurs sans doute pas autrement que par l'existence d'un réseau de clientèles dont la base est très vraisemblablement urbaine.

Le rapport clientélaire, particulièrement aux $\mathrm{II}^{\mathrm{e}}$ et $\mathrm{III}^{\mathrm{e}} \mathrm{s}$., a pour motif essentiel l'ostentation sociale du patron qui assure sa promotion à travers lui (Rouland, 1979, p. 566). L'existence de clients, raisonnablement induite des pratiques

26. Ainsi, les charges et évergésies que les incolae étaient tenus d'effectuer dans leur cité d'accueil avant de pouvoir être intégrés au corps décurional pourraient-elles, par exemple, être considérées comme autant d'étapes d'acquisition de cette dignitas, nécessaire au statut visé. Sur ces incolae, voir Arnaud, 1999, spécialement p. 43-47. 
communes à tout l'Empire, de rares allusions écrites et de l'interprétation que l'on fait des maisons à péristyle, ne serait toutefois guère décelable à travers des éléments matériels précis: peut-être les quelques larges vestibules bipartites précédés d'un porche (de type vestibulum-fauces) ou la longue banquette rouge en maçonnerie installée sous le portique de façade, juste à côté du porche de la Maison à l'Opus Quadratum à Limoges (Loustaud, 2000, p. 196, fig. 46) (fig. 4).

Toutes ces circonstances et évolutions ont fait que la domus est devenue toujours un peu plus un instrument de manifestation de pouvoir individuel. Progressivement, avec l'enrichissement lié à la Pax Romana et aux progrès de la romanisation, les vestiges archéologiques mettent alors en évidence, parallèlement à la hausse des évergésies, une surenchère dans la taille quelquefois, mais surtout dans le luxe des maisons. Au sein d'une ostentation employant une gamme assez standard, et donc impersonnelle, de moyens, une hiérarchisation semble cependant s'effectuer par l'usage de formes architecturales et décoratives (souvent empruntées à l'architecture publique) ou de techniques dont la qualité et la quantité font la différence entre les maisons. Avec le temps, apparaissent ou se développent de plus en plus des éléments personnels, intimement mêlés aux éléments «classiques »: scènes de loyalisme impérial mettant ou non en scène le maître, commémoration d'évergésies, représentations de ses passions (sportives ou littéraires). Tous ces éléments qui renseignent les visiteurs sur la vie du maître des lieux jouent un rôle important dans sa promotion.

\section{LES DOMUS DES ÉLITES EN GAULE : FORMES ET SIGNIFICATION}

\section{L'INTRODUGTION DE LA MAISON À PÉRISTYLE EN GAULE}

Dans cette optique, les maisons à péristyle édifiées à partir de l'époque augustéenne dans les chefs-lieux de cités auraient été introduites pour des raisons politiques. En favorisant les élites indigènes et en s'efforçant de les associer étroitement à la gestion des provinces, la politique impériale a exporté des mécanismes de pouvoir qui, en l'absence de structures locales adaptables à leur pratique, ont également nécessité d'importer le principal instrument qui permettait leur fonctionnement. Toutefois, son adoption et son adaptation ont varié en fonction de l'époque et des moyens dont ces élites disposaient.

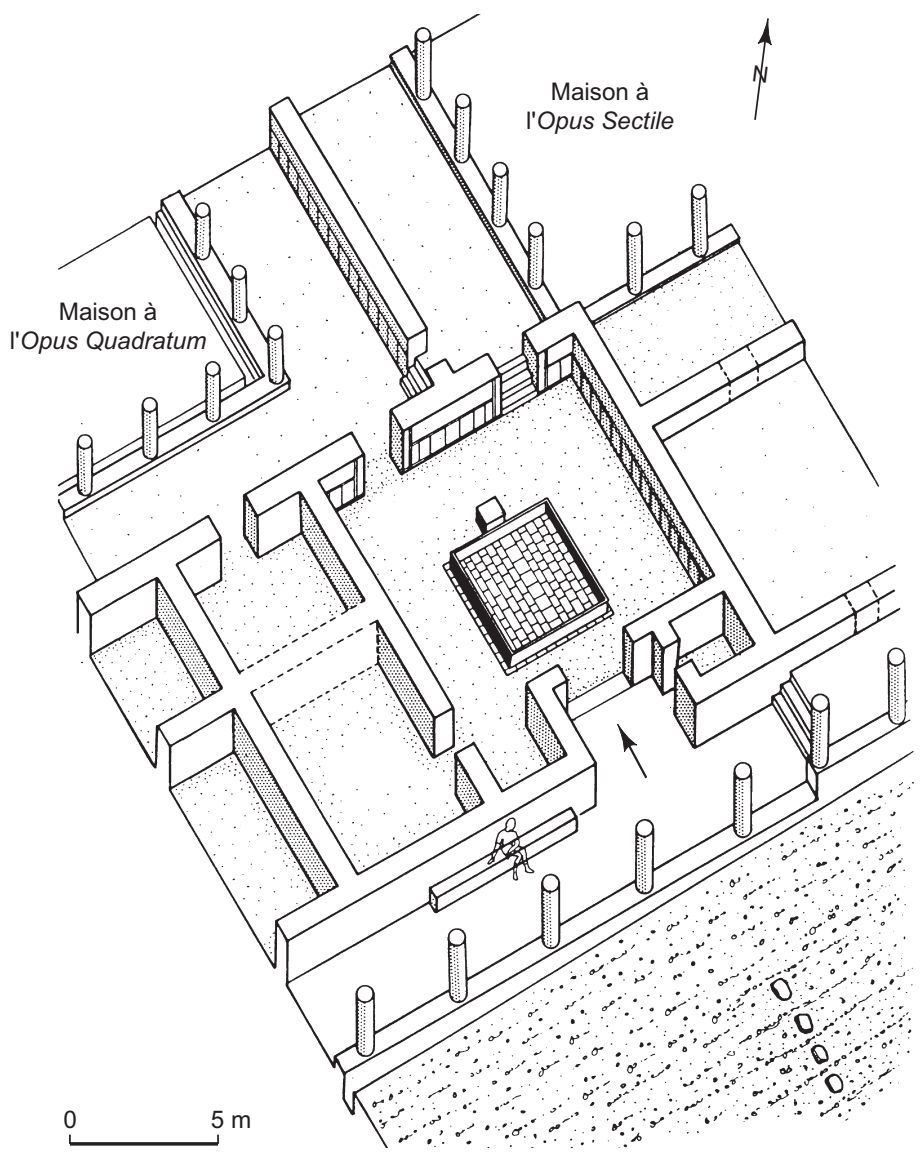

Fig. 4 - Entrée des Maisons à l'Opus Quadratum et à l'Opus Sectile à Limoges (Haute-Vienne) (d'après Loustaud, 2000, fig. 46).

Ce qui a été introduit, c'est le type d'habitat de la classe dominante romaine, modèle où se mêlent influences grecques et romaines ${ }^{27}$, mais dont il est d'ailleurs fort probable que les premiers Gaulois romanisés ne percevaient que la romanité. Hormis peut-être par l'emploi de certaines techniques architecturales 28 , les premières maisons apparues en Gaule n'ont en effet rien de provincial. Il ne s'agit cependant pas de modèles livrés clés en

27. Sur la part des antécédents hellénistiques et italiques dans l'architecture de la Gaule du Sud, et sur les modèles architecturaux hellénistiques utilisés comme porteurs des valeurs romaines, voir Gros, 2001, p. 142-147. La finalité de la recherche de l'hellénicité ou l'italicité de tel ou tel caractère de la maison à péristyle d'époque impériale ne paraît donc pas devoir mériter l'intérêt souvent primordial que lui prêtent certains auteurs puisque toutes deux participent chacune autant de la romanité.

28. Le premier état de la Maison à péristyle (ou schola?) du Verbe Incarné, à Lyon, est en adobe ou à pans de bois, alors que le décor peint de qualité ne montre, lui, pas de décalage avec l'Italie (Desbat, Mandy, 1991, p. 97). Voir également les exemples et la discussion dans Paunier et al., 2002. 
main comme le montre l'apparente diversité des plans, caractéristiques des modèles italiens contemporains ${ }^{29}$. Avec les progrès de la romanisation et l'intensification des contacts entre les notables, la piste d'éventuels modèles est complètement brouillée, mais l'uniformisation de ces maisons (par le biais du décor et du luxe essentiellement) s'accroît toujours un peu plus, en collant au plus près aux préceptes de l'époque augustéenne théorisés par Vitruve, apparemment sans trop s'attacher aux contraintes normatives comme en témoignent les plans retrouvés ou la littérature de vulgarisation ${ }^{30}$.

Plus qu'à l'histoire politique de la Gaule, l'apparition des maisons à péristyle est liée à la création ou au développement des villes à l'époque augustéenne. On constate en effet partout l'écoulement d'un certain délai entre la conquête et l'apparition des premiers cas.

C'est un fait que les élites (autochtones ou immigrés italiens), qui ont dû avoir besoin de (re)constituer et de consolider leur fortune, n'avaient peut-être pas au début les moyens d'assumer les frais de construction et de fonctionnement de telles maisons, mais devaient également quelquefois comprendre l'utilisation qu'elles pouvaient en faire et les avantages qu'elles pouvaient en tirer. Mais ce n'est pas la seule raison, pour le Midi du moins, depuis longtemps conquis, urbanisé et sous influence grecque ${ }^{31}$, où les maisons à péristyle riches, d'aspect tout à fait romain, apparaissent bien longtemps après la conquête de la fin $\mathrm{du} \mathrm{II}^{\mathrm{e}}$ s. av. J.-C. en Gaule Transalpine et, souvent, avec un certain décalage après la création augustéenne de la province de Narbonnaise en 25 av. J.-C. On note cependant des cas contemporains ou légèrement postérieurs à cette dernière ${ }^{32}$.

29. Il suffit de regarder la Maison du Clos de la Lombarde à Narbonne ou celle des Nones de Mars à Limoges (notamment leur décor peint) pour s'en convaincre.

30. Les proportions des espaces sont, par exemple, complétement absentes du manuel de M. Cetius Faventinus, abrégé de Vitruve et compilation d'autres auteurs non identifiés, datable de la première moitié du $\mathrm{III}^{\mathrm{e}} \mathrm{s}$. (ou peut-être du IV $\mathrm{e}$.) et s'adressant aux propriétaires de domus (Cam, 2001, p. 101).

31. Il est cependant loin d'être sûr que les quelques maisons à péristyle de type grec connues (en particulier à Glanum) aient eu une influence notable sur le modèle d'époque impériale.

32. Peut-être dès la fin de la République à Narbonne même (colonie depuis 118 av. J.-C., mais capitale en 22 av. J.-C.), au Clos de la Lombarde; dès la fin du $\mathrm{I}^{\mathrm{er}} \mathrm{s}$. av. J.-C. à Fréjus; dès les années 2030 apr. J.-C. à Nîmes ; également au début du $\mathrm{I}^{\text {er }}$ s. sans doute aussi à Aix-en-Provence, mais seulement dans les années 40 à Vienne ; au milieu ou dans le courant du I ${ }^{\mathrm{er}}$ s. apr. J.-C. à Orange ; dans la seconde moitié du I ${ }^{\text {er }}$ s. apr. J.-C. à Vaison-la-Romaine.
Les raisons d'un tel décalage ne sauraient s'expliquer par l'absence d'urbanisation (comme dans les Trois Gaules) ou d'un manque d'argent qui n'auraient pas permis d'adopter une maison depuis longtemps représentative d'un art de vivre romain. Peut-être devrait-on également supposer, en admettant qu'une telle maison soit en quelque sorte le résultat d'une dignitas acquise, que les élites n'aient pas encore été jugées prêtes ou que la nécessité sociale ne s'en soit pas fait sentir, mais il faut sans doute plutôt y voir des raisons historiques. En effet, durant la majeure partie du $\mathrm{I}^{\mathrm{er}}$ s. av. J.-C., à Rome même, ce type de maison est en train de s'illustrer dans les luttes aristocratiques qui secouent la Ville ${ }^{33}$ et la mise en évidence du pouvoir qu'elle représente pourrait avoir incité le Sénat à ne pas en favoriser la diffusion dans une province non entièrement pacifiée.

La situation est encore la même lors de la conquête de la Gaule Chevelue entre 58 et 52 av. J.-C. et l'apparition de la plupart des maisons aristocratiques intervient assez longtemps et de manière extrêmement variable d'une cité à l'autre. La présence d'Auguste à Lyon, de 16 à 13 av. J.-C., pour organiser les provinces et les cités en donnant un rôle important aux chefs-lieux a sans doute joué un rôle capital pour initier le mouvement et ce n'est sans doute pas un hasard si c'est précisément à Lyon, capitale de la nouvelle province de Lyonnaise, qu'on en constate l'existence, dès l'aménagement du sanctuaire impérial à l'époque augustéenne, avec la Maison du Verbe Incarné (Desbat, Mandy, 1991, p. 97). Certains cas plus précoces (vers 30 av. J.-C.) sont même attestés sur l'oppidum de Bibracte ${ }^{34}$, chef-lieu des alliés Éduens ; situation qui pourrait bien être due aux rapports privilégiés entretenus par les autochtones avec Rome depuis le $\mathrm{II}^{\mathrm{e}} \mathrm{s}$. av. J.-C. (fig. 5).

Contrairement au cas de la Narbonnaise, la conquête de la Gaule Chevelue - où n'existait pas d'antécédent urbain -, vers le milieu du ${ }^{\mathrm{er}}$ s. av. J.-C., coïncide à Rome avec une période de forte transformation de l'usage des maisons aristocratiques. Avec le décalage entre ce moment et les débuts effectifs de l'implantation des premières grandes domus à péristyle, souvent bien après l'époque augustéenne, c'est donc un modèle dominant déjà adapté aux nouvelles réalités politiques de l'Empire et dont l'adaptation à l'administration des provinces a déjà eu l'occasion d'être testée dans d'autres régions qui arrive en Gaule.

33. Ce n'est que dans les années 10 av. J.-C. que la fonction politique dévolue à la domus (rôle principalement clientélaire) prend l'aspect qu'on lui connaît au début de l'Empire (Guilhembet, 2001, p. 221).

34. En particulier les Maisons 1 et 2 du Parc aux Chevaux (Goudineau, 1993 ; Paunier, Luginbühl dir., 2004). 

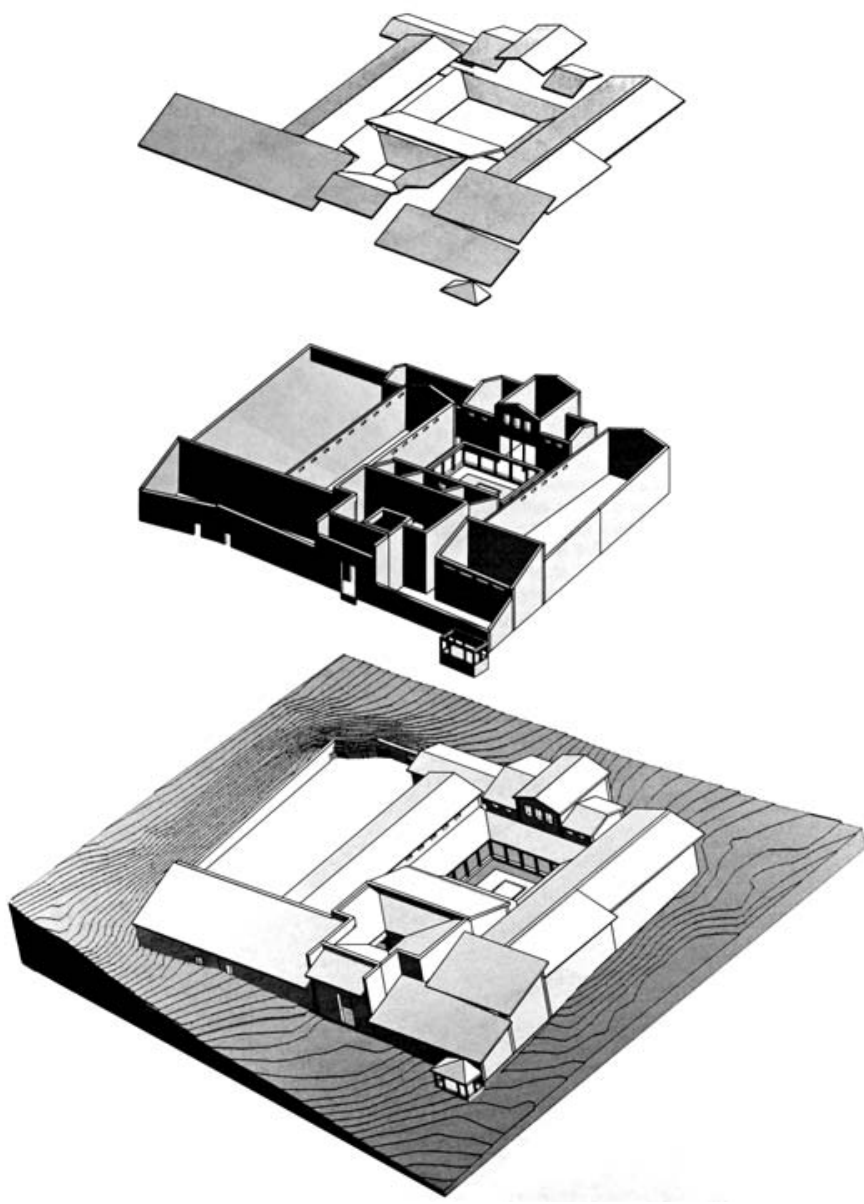

Fig. 5 - Une maison à péristyle des Trois Gaules précoce : la Maison PC 1 de Bibracte (Mont Beuvray), vers le changement d'ère (d'après Paunier, Luginbühl dir., 2004, p. 408-409).

Le cas lyonnais du Verbe Incarné pourrait laisser supposer un rôle de l'État dans l'introduction de ce modèle domestique, mais les preuves en font encore défaut et l'on ignore notamment le rôle des immigrants italiens, la nature et le nombre des étapes suivies par d'éventuels modèles avant d'être construits. Ailleurs dans les Trois Gaules, dans le meilleur des cas, les premières maisons à péristyle riches sont contemporaines de la réelle éclosion des villes sous Tibère et Claude, souvent contemporaines également des grands monuments publics et, comme eux, situées au plus près du centre politique et religieux et construites en pierre dès l'origine. On peut supposer que certains aristocrates (immigrés ou indigènes) étaient alors suffisamment riches pour en supporter le coût ou que l'État a accordé des aides, comme cela semble avoir été le cas en Bretagne où Tacite rapporte qu'en 79-80 (donc une génération après la conquête), pour favoriser l'assimilation des nouveaux conquis, le gouverneur de la province, Cn. Julius Agricola, « exhorte les particuliers, aide les cités à construire temples, marchés, maisons " 35 parmi lesquelles figuraient peutêtre des maisons à péristyle dont l'archéologie atteste les premiers exemples précisément à la fin du $\mathrm{I}^{\mathrm{er}} \mathrm{s}$. à Londres (Cottica, 1994, p. 95), cité prospère et promue capitale de la province dans les années 80-90. Ailleurs, malgré le dynamisme économique rapide de la province, on doit attendre le début du $\mathrm{II}^{\mathrm{e}} \mathrm{s}$. - à Leicester - ou le milieu de ce siècle - à Caerwent, Gloucester, Colchester - pour trouver des exemples, ce qui tendrait à montrer que le décalage entre la conquête et l'apparition des maisons à péristyle n'est pas un problème seulement financier, mais aussi mental. Il amène également à se poser la question des anciens lieux et modes de résidence des élites ; l'essentiel de leur activité pourrait s'être encore exercé dans le cadre rural et n'avoir été définitivement fixé en ville qu'à partir de l'obtention du statut juridique de celle-ci ou, pour les cités pérégrines, d'un développement matériel suffisamment avancé.

Tous ces constats, gaulois ou bretons, semblent aller dans le sens d'une démonstration de la liaison de la maison à péristyle urbaine plus avec l'exercice du pouvoir qu'avec une simple satisfaction de l'otium.

\section{LES COMPOSANTES FONDAMENTALES DES DOMUS DES ÉLITES GAULOISES}

Il paraît actuellement vain de chercher à proposer pour ces maisons une typologie, nécessairement insatisfaisante parce qu'établie sur trop peu de cas - souvent incomplets -, variable suivant les critères privilégiés et finalement d'un intérêt très relatif parce que ne prenant en compte que des critères de formes.

L'atypisme relatif de certaines composantes de ces maisons (cuisine, pièces de services aux fonctions mal définies, etc.), guère distinguables de celles de l'habitat courant, si ce n'est, quelquefois, par leurs dimensions ou leur qualité, n'en fait pas des critères d'étude pertinents.

On a déjà eu l'occasion d'évoquer le fait que, si le péristyle est l'élément le plus symbolique des maisons aisées, les demeures des élites urbaines ne sauraient se réduire à ce seul élément qui n'a qu'un rôle d'agrément quand il n'est pas associé étroitement à d'autres composantes, elles aussi

35. Tacite, Vie d'Agricola, 21, 1 : « [...] hortari privatim, adjuvare publice, ut templa, fora, domos exstruerent [...]»(H. Goelzer, Paris, Les Belles Lettres, 1922, p. 124-125). Les auteurs britanniques pensent généralement que cette assistance est plus d'ordre militaire (technique ou humaine) que financière (Blagg, 1984, p. 249-250). 
publiques, qui confèrent à la maison à péristyle son fort particularisme.

Si l'œuvre de Vitruve reste essentielle pour en comprendre la signification, il faut toutefois rappeler que, sous l'Empire, les témoignages écrits et les ambiguïtés du vocabulaire font apparaître une diversification accrue des pièces et notamment une tendance à l'indifférenciation des salles de réception, dont les usages ne sont pas uniquement liés à la représentation sociale (Callebat, 1999; Guilhembet, 2001, p. 231). En outre, les préceptes vitruviens régissant la construction de la maison ne sont que très rarement strictement appliqués à la lettre, mais sont au contraire sujets à cette adaptation qui apparaît tout au long du De Architectura et règne en maître sur la construction des maisons de Gaule. Qu'elles soient liées notamment aux changements politiques du régime romain, à des délais d'acquisition de pratiques sociales et culturelles (romanisation), aux conditions naturelles du sol (matériaux de construction locaux) et du climat, à des contraintes urbaines (topographie, place disponible, obstacles, législation), à des opportunités (agrandissements de lots, disponibilité et qualité du faber aedium) ou à des conditions socio-économiques locales, ces adaptations, multiples et le plus souvent interdépendantes, expliquent qu'à partir d'un schéma structurel finalement assez simple on arrive à des résultats très variés sur le plan morphologique. La réelle uniformité des domus à péristyle provinciales est en fait due moins à la présence et à la disposition strictes d'un certain nombre de pièces - notamment publiques, obligatoires, mais en nombre et de formes variables - et de divers éléments caractéristiques, qu'au maintien d'une circulation conservant une hiérarchie entre les espaces.

L'étude croisée d'un certain nombre d'éléments, relatifs aussi bien aux plans disponibles, aux structures, à l'organisation ou au fonctionnement des constructions, qu'à la genèse ou à l'environnement des bâtiments, fait effectivement ressortir un faisceau de composantes qui sont caractéristiques et souvent fondamentales pour définir ce que sont précisément ces maisons de la frange supérieure de la population citadine ${ }^{36}$.

La plus essentielle, celle sur laquelle s'appuient en fait la plupart des autres, est constituée par une trilogie architecturale, structurelle et fonctionnelle, formée par une large entrée, une ou plusieurs salles de réception et le péristyle autour duquel les premières s'articulent. La légitimité de l'importance accordée à l'association de ces trois

36. C'est ce qui explique d'ailleurs l'atypisme souvent assez poussé des maisons humbles où l'absence totale de parties publiques ne joue pas de rôle aussi structurant. éléments repose sur la lecture de ces structures à travers l'œuvre de Vitruve, tout particulièrement le passage cité plus haut où il aborde le problème de l'adéquation de la maison au statut social du propriétaire et surtout à travers les conclusions qu'en ont tirées A. Wallace-Hadrill (1988) et F. Coarelli (1989) sur la structure sociale de la maison romaine italienne de l'élite aristocratique de la fin de la République et du début de l'Empire. Ces travaux novateurs ont remarquablement contribué à mettre en évidence et à faire comprendre les mécanismes de fonctionnement des demeures des puissants et à permettre de dépasser une étude jusqu'alors trop strictement axée sur l'aspect architectural et formel de l'œuvre-clé de Vitruve. Leurs remarques et conclusions paraissent, grosso modo, pouvoir s'appliquer à l'habitat des élites provinciales de l'ensemble de l'Empire qui ont naturellement calqué leur comportement social sur ce modèle dominant ${ }^{37}$.

Dans ce système (la théorie des axes de différenciation sociale), c'est davantage la présence de certains éléments architecturaux que leur stricte disposition les uns par rapport aux autres qui joue un rôle dans le fonctionnement de la maison. Ce schéma de fonctionnement domestique ne concerne pas que les pièces de la «trilogie », mais comme celles-ci en constituent néanmoins le noyau dur, il convient donc de s'y arrêter un instant pour mieux en comprendre la signification.

Les riches Romains ayant une vie très publique, peu d'endroits étaient réellement privés dans une domus où circulaient des esclaves ou des visiteurs ayant une vue à partir du péristyle sur presque toute la maison. Vitruve distingue cependant clairement les parties privées des publiques et la conception romaine de la vie sociale admettait de nombreuses et subtiles gradations dans le degré de la vie privée d'une pièce par rapport à une autre ; elles variaient suivant les besoins et le goût de chaque propriétaire, suivant les heures de la journée et suivant la présence ou l'absence d'étrangers dans la maison. De plus, les Romains ne connaissant pas notre distinction entre espaces de travail et de loisir, tout se faisait chez soi et la distinction negotium/otium correspondait, dans le cadre domestique, à une question de temps, globalement entre le matin et l'après-midi.

Pour compliquer encore cette situation, il faut prendre en considération l'existence, en plus de cette opposition public-privé, d'un axe de différenciation humble-grand qui permettait aux trois principaux groupes circulant dans une maison - à savoir la famille du propriétaire et sa

37. Leur validité a notamment été montrée pour les maisons d'Afrique (Thébert, 1985 et 1993). 
domesticité d'un côté et ses amici, clientes et autres relations d'un autre côté - de distinguer les espaces qui leur étaient réservés selon leur position sociale. Ces distinctions, dont une grande partie des règles devaient être acquises par l'éducation, ne sont plus guère décelables aujourd'hui qu'à travers ce qui subsiste de signaux faisant appel aussi bien à des formes architecturales ou au décor (notamment à des codes de couleurs), qu'à une série non limitative de moyens humains (personnel spécialisé : ostiarius, cubicularii, nomenclatores...), physiques, comme des portes ou des écrans mobiles (tentures, paravents) ou des différences de hauteur des sols. Pour les éléments de la trilogie, la circulation de l'un à l'autre devait être assurée par les mêmes signaux (particulièrement concentrés dans ces espaces) et sans doute également par des habitudes rituelles.

Cette double opposition entre le public et le privé d'un côté, et les humbles et les grands de l'autre, jointe à la disparition de la majeure partie de ces signaux (ou du code permettant de les comprendre), explique les difficultés des archéologues, confrontés au dépouillement des ruines exhumées, à comprendre clairement l'organisation d'une maison. Encore une fois, on pressent cependant que, si règles il y a eu, des variations individuelles ou locales n'ont pas dû manquer, mais, comme le montrent des études de cas, assez exceptionnellement documentés (Vipard, 1996 et 2001, par exemple) il n'en est pas moins vrai que la validité de ces schémas de fonctionnement en Gaule peut être tenue pour une quasi-certitude.

Mutatis mutandis, on arrive à montrer qu'une lecture vitruvienne des maisons des élites reste toujours possible et même nécessaire, sans qu'il y ait besoin de trop solliciter les documents.

Outre la trilogie publique, les autres composantes caractéristiques des maisons qui nous intéressent ne sont sans doute pas toutes identifiées ou documentées faute d'avoir laissé des indices archéologiques ou textuels suffisants, mais parmi les plus importantes, intimement liées d'ailleurs à cette trilogie et au rôle social de la maison, il semble que l'on puisse faire entrer: la décoration (avec, notamment, ses caractères publics) et le luxe, la dimension des bâtiments et son fréquent corollaire, l'instabilité des plans, ainsi qu'un certain nombre d'éléments (essentiellement de confort) dont l'importance réelle est moins bien définie. Il faut également tenir compte de la prédominance de certaines liaisons avec l'environnement urbain lui-même (problème qui sera abordé plus loin) et, enfin, tenir compte du rôle de l'adaptation dans l'organisation de toutes ces caractéristiques.
Ce sont ces différents éléments, généralement communs à la majeure partie des maisons à péristyle de l'Empire, que l'on voudrait faire ressortir à travers les cas gaulois en essayant de suggérer d'éventuelles possibilités de variations et de hiérarchisation entre elles.

\section{LA TRILOGIE PUBLIQUE}

\section{Le péristyle}

Quoiqu'il ne soit sans doute pas plus important que les deux autres éléments dans la trilogie, le péristyle n'en reste pas moins le plus hautement symbolique de la romanité et ce n'est pas sans raison que c'est précisément cet aménagement qui sert à caractériser un des rares types de maisons se distinguant de la masse de l'habitat.

Qu'on lui attribue une valeur d'agrément ou une valeur sociale, sans que cette seconde conception soit exclusive, il désigne nettement des propriétaires appartenant à la frange supérieure de la population citadine.

Depuis son implantation dans les maisons italiques au III $^{\text {e }}$ s. av. J.-C. (Pesando, 1997, p. 267-274) et l'abandon progressif de l'atrium qu'il a entraîné, le péristyle est peu à peu devenu l'élément déterminant de la maison d'époque impériale et c'est le plus souvent une conception déjà évoluée qui s'implante en Gaule lors de la romanisation.

Dans les villes, où la place est chère, l'espace qu'il nécessite est le signe le plus immédiatement évident de son appartenance aux maisons riches. La valeur absolue ou relative de sa surface, qui est souvent l'une des seules données dont on dispose, est donc un critère de comparaison fréquemment utilisé entre les domus mais, sans en minimiser l'importance, il faut toutefois la relativiser en prenant en considération - ce qui est toujours délicat - la qualité de sa réalisation ou le rôle pondérateur du luxe dans l'appréhension par ses contemporains de la majesté de cet aménagement. La valeur tant symbolique que politique du péristyle en faisait un élément de choix dans l'ostentation et dans la rivalité entre notables, et c'est sans doute ce qui explique les grandes variations dont on peut quelquefois constater l'existence à travers les bribes d'informations parvenues jusqu'à nous.

Même si les chiffres ne peuvent rendre compte que très imparfaitement de la perception subjective que les contemporains pouvaient avoir de cet espace, les données métriques constituent un critère dont on ne peut se passer. Quelques mesures suffisent à bien montrer la place occupée par cet aménagement dans un certain nombre de maisons gauloises et dispensent de longs commentaires (tabl. I). 
Tabl. I - Dimensions de quelques péristyles de maisons gauloises et bretonnes, classées par rapport au pourcentage occupé dans la maison: 1, surface du péristyle en mètres carrés ; 2, pourcentage par rapport à la surface de la maison ; 3, surface de l'aire couverte en mètres carrés ; 4, pourcentage par rapport au péristyle; 5, surface de l'aire découverte en mètres carrés ; 6, pourcentage par rapport au péristyle; 7 , rapport aire couverte/aire découverte.

\begin{tabular}{|c|c|c|c|c|c|c|c|c|}
\hline Localisation & Dénomination & 1 & 2 & 3 & 4 & 5 & 6 & 7 \\
\hline Vaison-la-Romaine (Vaucluse) & Maison au Buste d'Argent, 3 péristyles ( $3500 \mathrm{~m}^{2}$ minimum) & 2057 & 58,8 & 887 & 43,1 & 1170 & 56,9 & 10,3 \\
\hline Saint-Romain-en-Gal (Rhône) & Maison sous le Lycée (4 $600 \mathrm{~m}^{2}$ au moins) & 2260 & 49,1 & 837 & 37 & 1423 & 63 & 10,7 \\
\hline Saint-Romain-en-Gal (Rhône) & Maison des Dieux Océan, état 3A, 2 péristyles (2932 m²) & 1408 & 48 & 566 & 40,2 & 842 & 59,8 & 10,5 \\
\hline Saint-Romain-en-Gal (Rhône) & Maison des Dieux Océan, états 3B et 4 (surface indeterminée) & 1408 & 48 & 613 & 43,5 & 795 & 56,5 & 10,3 \\
\hline Lyon (Rhône) & Maison aux Xenia, péristyle II, phase 2 (660 m² environ) & 306 & 46,4 & 181 & 59,2 & 125 & 40,8 & 0,7 \\
\hline Rezé (Loire-Atlantique) & Maison sud du Terrain Peigné $\left(880 \mathrm{~m}^{2}\right)$ & 380 & 43,2 & 185 & 48,7 & 195 & 51,3 & 10,1 \\
\hline Vaison-la-Romaine (Vaucluse) & Maison au Dauphin, état 3, 2 péristyles $\left(2700 \mathrm{~m}^{2}\right)$ & 1130 & 41,9 & 305 & 27 & 825 & 73 & 20,7 \\
\hline Saint-Romain-en-Gal (Rhône) & Maison des Dieux Océan, état 2B, 2 péristyles $\left(2760 \mathrm{~m}^{2}\right)$ & 1135 & 41,1 & 437 & 38,5 & 698 & 61,5 & 10,6 \\
\hline Amiens (Somme) & Maison 1 du Palais des Sports $\left(465 \mathrm{~m}^{2}\right)$ & 190 & 40,9 & 90 & 47,4 & 100 & 52,6 & 10,1 \\
\hline Saint-Romain-en-Gal (Rhône) & Maison des Dieux Océan, état 2A, 2 péristyles (1 326 m²) & 531 & 40 & 173 & 32,6 & 358 & 67,4 & 20,1 \\
\hline Périgueux (Dordogne) & Maison de la rue des Bouquets (2 $350 \mathrm{~m}^{2}$ au moins) & 913,5 & 38,9 & 409,5 & 44,8 & 504 & 55,2 & 10,2 \\
\hline Limoges (Haute-Vienne) & Maison des Nones de Mars, 2 péristyles $\left(3735 \mathrm{~m}^{2}\right)$ & 1395 & 37,3 & 675 & 48,4 & 720 & 51,6 & 10,1 \\
\hline Wroxeter (Grande-Bretagne) & Maison à Corridor VI (1 $\left.615 \mathrm{~m}^{2}\right)$ & 577 & 35,7 & 252 & 43,7 & 325 & 56,3 & 10,3 \\
\hline Saint-Romain-en-Gal (Rhône) & Maison aux Cinq Mosaïques $\left(500 \mathrm{~m}^{2}\right)$ & 157,5 & 31,5 & 77,5 & 49,2 & 80 & 50,8 & 1 \\
\hline Fréjus (Var) & Maison du Clos de la Tour (1 $090 \mathrm{~m}^{2}$ environ) & 330 & 30,3 & 143 & 43,3 & 187 & 56,7 & 10,3 \\
\hline Narbonne (Aude) & Maison du Clos de la Lombarde $\left(975 \mathrm{~m}^{2}\right)$ & 292,5 & 30 & 191,5 & 65,5 & 101 & 34,5 & 0,5 \\
\hline Alba-la-Romaine (Ardèche) & Maison du Champ Delauzun (1 $420 \mathrm{~m}^{2}$ minimum) & 390 & 27,5 & 235 & 60,3 & 155 & 39,7 & 0,7 \\
\hline Saint-Romain-en-Gal (Rhône) & Maison au Lion (3 $100 \mathrm{~m}^{2}$ environ) & 850 & 27,4 & 436 & 51,3 & 414 & 48,7 & 0,9 \\
\hline Caerwent (Grande-Bretagne) & Maison à Corridor de l'Insula VII (1 $\left.620 \mathrm{~m}^{2}\right)$ & 425 & 26,2 & 196 & 46,1 & 229 & 53,9 & 10,2 \\
\hline Caerwent (Grande-Bretagne) & Maison de l'Insula IIIS (1 980 m²) $^{2}$ & 512,5 & 25,9 & 386,5 & 75,4 & 126 & 24,6 & 0,3 \\
\hline Metz (Moselle) & 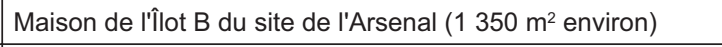 & 324 & 24 & 144 & 44,4 & 180 & 55,6 & 10,3 \\
\hline Périgueux (Dordogne) & Maison à l'Impluvium Polylobé (plus de 2500 m²) & 598 & 23,9 & 377 & 63 & 221 & 37 & 0,6 \\
\hline Avenches (Suisse) & Maison de l'Insula XVI Est (1 570 m² environ) $^{2}$ & 360 & 22,9 & 206,5 & 57,4 & 153,5 & 42,6 & 0,7 \\
\hline Vieux (Calvados) & Maison au Grand Péristyle (1 $\left.421 \mathrm{~m}^{2}\right)$ & 322,4 & 22,7 & 192 & 59,6 & 130,4 & 40,4 & 0,7 \\
\hline Cologne (Allemagne) & Maison à Atrium de la Cathédrale $\left(1440 \mathrm{~m}^{2}\right)$ & 313,5 & 21,8 & 173,5 & 55,3 & 140 & 44,7 & 0,8 \\
\hline Bibracte (Saône-et-Loire) & Maison PC $2\left(1040 \mathrm{~m}^{2}\right)$ & 225 & 21,6 & 169 & 75,1 & 56 & 24,9 & 0,3 \\
\hline Saint-Raphaël (Var) & Maison du Suveret $\left(460 \mathrm{~m}^{2}\right)$ & 94,5 & 20,5 & 82,3 & 87 & 12,3 & 13 & 0,1 \\
\hline Saint-Romain-en-Gal (Rhône) & Maison des Dieux Océan, états 2A et 2B (1326 m²) & 200 & 15,1 & 118 & 59 & 82 & 41 & 0,7 \\
\hline Vaison-la-Romaine (Vaucluse) & Maison au Dauphin, états 3 et $4\left(2700 \mathrm{~m}^{2}\right)$ & 378 & 14 & 274,5 & 72,6 & 103,5 & 27,4 & 0,4 \\
\hline Limoges (Haute-Vienne) & Maison des Nones de Mars (3 $\left.735 \mathrm{~m}^{2}\right)$ & 512,5 & 13,7 & 318,5 & 62,1 & 194 & 37,9 & 0,6 \\
\hline Saint-Romain-en-Gal (Rhône) & Maison des Dieux Océan, états 3 et 4 (2932 m²) & 388 & 13,2 & 221 & 57 & 167 & 43 & 0,8 \\
\hline Vieux (Calvados) & Maison au Petit Péristyle (1 $\left.421 \mathrm{~m}^{2}\right)$ & 166,8 & 11,7 & 124,9 & 74,9 & 41,9 & 25,1 & 0,3 \\
\hline
\end{tabular}

Les chiffres mettent en évidence quelques tendances ou apportent une confirmation objective à ce qui n'est souvent qu'une appréciation subjective. Il apparaît ainsi nettement, avec quelquefois de notables exceptions, que les plus grands péristyles (occupant un pourcentage élevé de la surface totale, 40 à $50 \%$ ou plus) sont associés aux plus grandes maisons et, du fait de la faible variété de largeur des aires couvertes (portiques), la surface centrale découverte y croît donc exponentiellement. Certaines maisons modestes se classent dans la catégorie la plus élevée dans le rapport aire découverte/aire couverte du fait de la présence, non d'un quadriportique, mais de portiques sur seulement un, deux ou trois côtés qui peuvent être interprétés comme une volonté de privilégier l'espace non couvert au détriment de la circulation (existence de culs-de-sac). En Gaule, sous l'Empire, il n'y a apparemment pas d'indice chronologique à tirer du nombre de portiques. Les plus simples (en U ou en $\mathrm{L}$ ) paraissent en revanche avoir un rapport avec les domus 
de taille modeste ou moyenne ${ }^{38}$, et les péristyles avec les plus grandes.

La qualité de réalisation de chaque péristyle semble avoir été très variable suivant les régions ou les capacités financières des propriétaires. En sus de la décoration vivante et bruyante créée par les végétaux, les animaux de passage (ou captifs ?) et les jeux d'eau, il faut également tenir compte de l'importance, sans doute non négligeable, des formes de décor plus classiques : les peintures sur les murs des portiques, mais aussi sur les colonnes et les monuments $\mathrm{du}$ jardin. Comme ailleurs dans l'Empire, on distingue une prédilection particulière pour les décors de jardins en trompe-l'œil qui contribuent à élargir les limites matérielles de l'hortus et, allant dans le même sens d'une intrication de la réalité et de la fiction, le recours à une faune fictive, réaliste ou idéalisée, vernaculaire ou exotique, et le plus souvent aviaire ou aquatique. De très beaux exemples de cette dernière, en rapport avec le support qu'ils ornent, existent notamment sur des bassins de la Maison de la rue des Bouquets à Périgueux (milieu du $\mathrm{II}^{\mathrm{e}} \mathrm{s}$.) et de la Maison au Grand Péristyle à Vieux (début du $\mathrm{III}^{\mathrm{e}}$ s.) ${ }^{39}$.

Un des éléments qui devait fortement contribuer à établir une distinction entre les domus, à savoir la statuaire spécifique à cet espace, si abondante et variée dans les maisons campaniennes, est presque inconnu en Gaule, même en Narbonnaise, sans doute moins du fait d'une réelle rareté que des déménagements et des récupérations : on en retrouve à peine quelques vestiges à Narbonne ${ }^{40}$, à Vaison-la-Romaine ou à Vienne (Jockey, 1996) - notamment les restes d'un Apollon archer et d'un groupe de Vénus et Amour dans le jardin de la Maison viennoise de l'Atrium (Collectif, 1996b, p. 419) -, quelques fragments très lacunaires de petits personnages et d'animaux à Vieux. Seul un lot un peu plus consistant, constitué d'une dizaine d'éléments sculptés très mutilés de la fin du $\mathrm{I}^{\mathrm{er}} \mathrm{s}$. ou du début du $\mathrm{II}^{\mathrm{e}}$ s., est connu à Saintes, sur le site de "Ma Maison », abandonné vers le milieu du $\mathrm{II}^{\mathrm{e}} \mathrm{s}$., dans les niveaux de destruction du péristyle. Il laisse entrevoir la coexistence d'œuvres de qualités et de provenances très diverses : un

38. Les cas plus anciens ne semblent pas en rapport avec la taille. Voir notamment le grand péristyle en U de la partie nord-est de la domus Augusta, construite sur le Palatin entre 36 et 29 (McKay, 1975, p. 70-71, par exemple).

39. Voir Barrière, Sarradet, 1996, p. 58-60 et Vipard, 2001, p. 26-27. D'autres cas sont attestés à Limoges et à Lisieux (Barbet et al., 1996, $\left.\mathrm{n}^{\mathrm{os}} 18-19\right)$.

40. Quelques fragments dispersés dans des niveaux de destruction pourraient éventuellement provenir de la Maison du Clos de la Lombarde parmi ceux publiés par Marcadé $e t$ al. en 1999. oscillum $^{41}$, des restes de statues d'un adolescent versant l'eau d'une cruche et d'un Jupiter, un hermès de Dionysos (sans doute d'origine italienne) et un groupe de deux divinités assises ainsi que deux autres, isolées et inachevées, qui proviennent d'un atelier local (Maurin dir., 1988, p. 279 et p. 288-289).

Un autre point fort de cette décoration résidait dans la concentration de colonnes dont il ne reste aussi que peu de traces. Les colonnes monolithes en marbre paraissent avoir été rarissimes et le développement et le succès du péristyle en Gaule semblent avoir été dus à l'usage généralisé des colonnes appareillées, en briques stuquées ou en roches locales. L'usage de la peinture et, quelquefois, dans la partie nord-occidentale et occidentale de la Gaule (Rouen, Vieux, Bourges, Saintes), d'un décor floral ou dionysiaque en faible relief, surtout dans la seconde moitié du $\mathrm{II}^{\mathrm{e}} \mathrm{s}$. et au début du $\mathrm{III}^{\mathrm{e}}$ s., d'inspiration publique, ont dû fortement contribuer à rehausser la magnificence du lieu. L'emploi du péristyle rhodien (Vitruve, De Architectura, VI, 7, 3) qui conférait au côté (nord) du portique qui en était doté une majesté immédiatement perceptible paraît avoir été l'apanage de certaines très grandes et belles maisons. Son existence est clairement attestée par l'emploi de colonnes de diamètres différents, géminées ${ }^{42}$ ou simples, et, dans ce dernier cas, souvent plus luxueuses ${ }^{43}$.

Sans doute très importante également pour l'aspect et la décoration, la végétation reste malheureusement une des grandes inconnues en Gaule. On ne sait pour ainsi dire rien de précis sur sa nature ou sa disposition, sinon qu'elle existait dans la majeure partie des viridaria. La présence d'un jardin (hortus) et non d'une cour ${ }^{44}$ - voire le remplacement fréquent de celle-ci par celui-là (mais jamais l'inverse) - paraît en effet être un caractère distinctif des

41. D'autres oscilla sont également attestés à Lyon, dans la Maison aux Masques (Desbat, 1984).

42. Par exemple, à Aix-en-Provence, dans la Maison du Jardin de Grassi ou à Vaison-la-Romaine, dans la Maison des Messii (Benoît, 1947, p. 101-102, 107 et fig. 7, p. 108).

43. Voir les colonnes lisses de $3 \mathrm{~m}$ et $2 \mathrm{~m}$ de la Maison du Génie Privé à Martigny (Paunier, 1996, p. 112-113), les cinq colonnes cannelées du portique nord de la Maison à l'Opus Quadratum de Limoges (Loustaud, 2000, p. 195, fig. 44) ou les deux colonnes à décor dionysiaque de l'aile nord de la Maison au Grand Péristyle, à Vieux (Vipard, 2001, p. 25-26).

44. On oppose souvent les deux types en considérant le premier comme grec, le second comme typiquement romain. Le dernier point est vrai, mais la cour dallée ou cimentée que l'on trouve dans les maisons romaines, si elle rappelle certains modèles grecs et peut s'en inspirer, a cependant la même fonction que le jardin. Son sol se trouve d'ailleurs fréquemment, comme ce dernier, en contrebas des portiques (ce qui n'est pas le cas dans la maison grecque). 


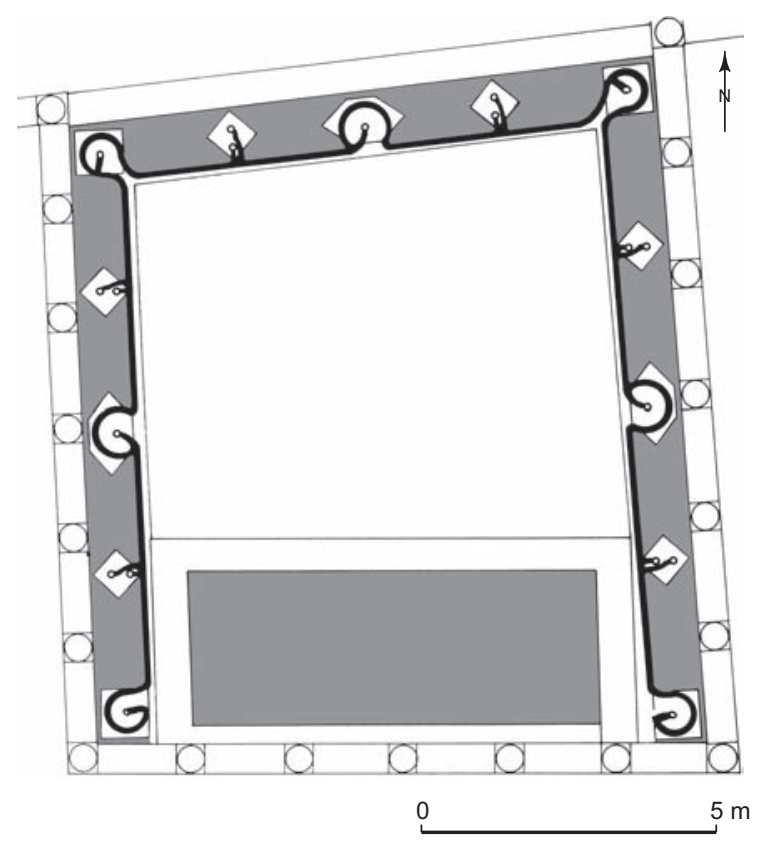

Fig. 6 - Bassin en U avec jets d'eau (en plomb) et bassin rectangulaire accolé au côté sud d'un des péristyles de la Maison au Vestibule à Colonnes à Saint-Romain-en-Gal (Rhône), vers 70 apr. J.-C. (d'après Desbat et al., 1994, fig. 134, p. 164).

plus grandes domus de Gaule, mais l'on ne peut être sûr que le décor et l'usage de jardinières n'aient pas contribué à réduire la différence entre les deux types. Ce qui importait, c'était sans doute plus la notion et un semblant de jardin que sa réalité matérielle.

La présence de l'eau, tout autant sinon plus que celle des végétaux, paraît être une caractéristique essentielle des maisons à péristyle et sans doute un élément de différenciation entre elles. Les manifestations les plus élaborées sont constituées par la présence de fontaines et de bassins divers (Dessales, 2003 et 2004).

Une étude typologique des divers bassins connus est limitée par les incertitudes de leur datation et la multiplicité de leurs formes ou de leurs emplacements : bassins quadrangulaires, circulaires ou polylobés, centraux, décentrés ou accolés à l'un des portiques, euripes (canaux découverts longeant les portiques). À l'heure actuelle, hormis ceux en forme de $\mathrm{U}$ (fig. 6), typiques de la région rhodanienne du $\mathrm{I}^{\mathrm{er}} \mathrm{s}$. au $\mathrm{III}^{\mathrm{e}} \mathrm{s}$. et qui semblent être une création provinciale originale ${ }^{45}$, aucun modèle n'apparaît comme particulièrement typique d'une époque ou d'une région donnée. La plupart renvoient cependant ouvertement à des modèles

45. Voir Desbat, 1991, p. 296 et Desbat et al., 1994, p. 198-199. italiens, et même souvent impériaux : à Nîmes, Orange, Vienne, Saint-Romain-en-Gal, par exemple, on rencontre très tôt le type rectangulaire avec une abside qui semble inspiré de celui de la villa de Livie à Rome et les bassins à niches plus ou moins complexes, inspirés des modèles impériaux du Palatin, se développent à partir du milieu du $\mathrm{I}^{\mathrm{er}} \mathrm{s}$. (Dessales, 2004, p. 23). Ces bassins polylobés se répandent partout dans l'Empire au II $^{\mathrm{e}}$ s. et au III $^{\mathrm{e}}$ s. (Grimal, Guey, 1937) ; les grandes possibilités de variation de la disposition et du nombre d'absides et d'arcs permettent un nombre infini de compositions qui font généralement de chaque exemplaire un cas unique; ils sont bien attestés en Gaule à toutes les époques sous des formes relativement simples, par exemple dans les Maisons du Jardin de Grassi à Aix-en-Provence, de l'Insula VI, 5 à Limoges, de la rue des Colonnes à Vienne (Lasfargues, 1982, p. 404) ou de la Visitation à Périgueux. La complexité des formes est une marque de l'évolution du type dans le temps (Maison de l'Impluvium Polylobé à Périgueux, Maison au Grand Péristyle à Vieux).

Certaines formes de bassins paraissent archaïques, comme l'euripe ou le bassin périphérique doté de jets d'eau de la Maison des Dieux Océan à Saint-Romain-en-Gal au $\mathrm{II}^{\mathrm{e}}$ s., de la Maison au Petit Péristyle (dernier quart du $\mathrm{II}^{\mathrm{e}} \mathrm{s}$.) ou de celle au Grand Péristyle à Vieux (début du $\mathrm{III}^{\mathrm{e}} \mathrm{s}$.) qui, en effet, se rencontre déjà sous une forme très voisine (avec les angles en quarts-de-rond typiques) dans les villae italiques de la seconde moitié du $\mathrm{I}^{\mathrm{er}} \mathrm{s}$. av. J.-C. ou encore en Campanie au milieu du I ${ }^{\text {er }}$ s. apr. J.-C. (Vipard, 1998, p. 55). L'Italie sert visiblement de modèle à la Gaule. On y retrouve les mêmes réminiscences et les tendances égyptisantes, comme avec le sphinx de la Maison viennoise des Dieux Océan (Dessales, 2004, p. 27-29).

Tous ces bassins et fontaines, dotés de jets d'eau, ne se conçoivent pas sans adduction sous pression. Même s'il en existe quelques cas anciens (par exemple, bassin de la cour de la Maison des Antes - d'inspiration hellénistique - de Glanum au $\mathrm{I}^{\mathrm{er}} \mathrm{s}$. av. J.-C.), leur développement est donc intimement lié aux programmes hydrauliques augustéens et postérieurs.

Malgré le silence des publications anciennes ou l'absence d'observations dans les fouilles plus récentes, cette adduction peut être supposée. Les tuyauteries en plomb ont en effet souvent été récupérées, tout comme, quelquefois, celles en bois, généralement identifiées grâce aux manchons en fer servant à les assembler ${ }^{46}$.

46. L’usage généralisé de tuyaux de chêne, de 1,50 m à 2,10 m de long environ, assemblés de force par des manchons en fer, est largement attesté dans les Trois Gaules, en Germanie et en Bretagne. Ils semblent 
À chaque fois que l'observation est possible (c'est-àdire seulement dans des fouilles récentes), la liaison entre l'adduction d'eau et la construction du bassin ressort bien de la concomitance de leur installation, mais également du fait que, sauf exception, cette eau courante ne sert jamais pour les besoins domestiques quotidiens (sauf, éventuellement, pour des bains) et que les puits ou citernes éventuels restent en usage après leur installation.

La présence de l'eau courante dans le cadre de vie adopté par la classe dirigeante répond en effet davantage à l'existence d'aménagements spécifiques de son environnement domestique (bassins, jets d'eau) et à un besoin de représentation sociale qu'à la volonté de satisfaire plus facilement les besoins essentiels aux fonctions quotidiennes ${ }^{47}$. La notion de commodité ou de confort semble bien s'être effacée derrière celle du plaisir et de l'ostentation, d'affirmation du pouvoir économique du maître.

À quelques exceptions près, une telle adduction domestique ne se rencontre d'ailleurs que dans les maisons pourvues d'un péristyle ${ }^{48}$. Le lien entre eau courante et jardin à péristyle est donc très fort et c'est un élément important pour déterminer la catégorie sociale de leur propriétaire.

Les conditions d'octroi de l'eau courante à des particuliers résidant en ville, moyennant un droit (vectigal) payé au trésor public, reste sans doute le point le plus mal étudié des recherches sur l'eau, tant en Italie que dans les provinces ${ }^{49}$. Dans ces dernières, l'attribution des concessions (aquaeductus) semble avoir été entre les mains des magistrats des cités et avoir également nécessité l'accord d'une grande partie de l'ordo ${ }^{50}$. Même si les démarches d'attribution demeurent très floues, il ressort que, pour les particuliers, l'obtention de l'eau à domicile devait être très chère et donc, de fait,

avoir fait l'objet d'une standardisation des modules analogue à celle des fistulae en plomb. Leur usage paraît avoir été complémentaire de ces dernières, utilisées de préférence dans les maçonneries et à l'extérieur.

47. Les puits et citernes existant dans les maisons mêmes, les fontaines publiques et le personnel assuraient la satisfaction de ces besoins.

48. Les maisons plus simples, mais généralement dotées d'une cour, pourraient alors être identifiées comme des résidences de décurions modestes.

49. Sur les divers développements de la recherche et des connaissances (avec bibliographie), voir Wilson, 1996, spécialement p. 23-24, et Koloski-Ostrow et al., 1997. L'article aquaeductus de E. Labatut, dans le Dictionnaire des Antiquités chrétiennes et de Liturgie, I, 1, 1877, p. 336345 , est encore très utile. Sur tous les problèmes concernant l'eau, voir aussi Hodge, 1992, en particulier p. 294-300 sur le raccordement des maisons urbaines au réseau public.

50. La lex municipalis d'Urso, en Bétique (ILS, 6087, cap. 100), indique, par exemple, que l'accord de 40 décurions est nécessaire à l'octroi de l'eau à un particulier. réservée à une élite économique; divers autres indices laissent même supposer que les membres de l'ordo devaient en être les bénéficiaires quasi exclusifs. C'est ainsi, par exemple, qu'une inscription italienne de 193 apr. J.-C. 51 indique qu'un sévir augustal reçoit du sénat local, en même temps que son fils accède au décurionat, le droit d'installer un conduit d'eau dans sa maison "comme s'il était décurion ». Outre cette précision fort instructive, ce texte montre que, même en Italie à la fin du $\mathrm{II}^{\mathrm{e}}$ s., le caractère honorifique et la vigilance accordés par les cités à l'attribution de l'eau à leurs concitoyens sont toujours intacts, et permet de supposer, même si la preuve écrite manque en Gaule, que la présence de l'eau courante dans une domus est un indice extrêmement fort en faveur de l'appartenance de son propriétaire à la classe décurionale, quel que soit par ailleurs le statut juridique ou économique de la cité.

Dans les villes de Gaule, on constate que, dès leur apparition, les premières maisons à péristyle dotées d'un jardin et de jets d'eau sont déjà équipées en eau courante (Maison B de la ZAC Villa Roma à Nîmes, vers 20 apr. J.-C., Maison aux Pierres Dorées de Saint-Romain-en-Gal, dès l'époque tibérienne, par exemple) et non de simples réservoirs.

L'étroite association entre maisons à péristyle et adduction d'eau revêt une telle nécessité que même si la création de ces dernières a pu créer le besoin, puis sa satisfaction, on est cependant conduit à se demander si elle ne serait pas plutôt subordonnée à l'existence préalable (ou concomitante) d'un réseau de distribution, sans lequel les divers jeux d'eau qui constituent l'une des caractéristiques essentielles de leur péristyle (cour et surtout jardin) ne peuvent exister. Cela pourrait notamment être l'une des raisons de la lenteur d'apparition de ce modèle domestique dans les parties les plus septentrionales. L'accroissement de leur nombre dans la seconde moitié du $\mathrm{I}^{\mathrm{er}} \mathrm{s}$. et au $\mathrm{II}^{\mathrm{e}} \mathrm{s}$. pourrait en effet plutôt être dû, non à un assouplissement des conditions d'octroi de l'eau aux particuliers, voire à l'éventuelle augmentation du nombre de gens susceptibles d'assumer sa charge financière, mais à la possibilité matérielle d'obtenir cette eau grâce à l'achèvement des réseaux de distribution d'eau (notamment en centre-ville).

Point central de la domus, lieu de passage obligé et point de focalisation des regards, le péristyle joue donc un rôle autant fonctionnel qu'esthétique ou social. Cette position privilégiée dans la maison explique sans doute que, quelles

51. CIL, X, 4760 = ILS, 6296, Suessa Aurunca : « [...] et ut aquae/digitus in domo ejus flueret com/modisque publicis ac si decurio fru/eretur [...] " (trad. : «[...] pour qu'un tuyau de dérivation d'eau coulât dans sa maison et qu'il jouît des avantages publics comme s'il était décurion [...]»). 
que soient les formules utilisées concernant le nombre des portiques, leur taille et leur luxe, on observe jusqu'au $\mathrm{III}^{\mathrm{e}}$ s., malgré une évidente inadaptation aux divers climats du nord-ouest de l'Empire, une volonté très nette de les agrandir, de les conserver, même sous la forme artificielle des portiques fenêtrés (Vipard, 2001-2002 et 2003) ${ }^{52}$.

\section{L'entrée}

Bien que son importance ne soit pas aussi immédiatement évidente que celle du péristyle pour les archéologues, l'espace d'entrée en revêt cependant une peut-être encore plus grande que ce dernier. C'est en effet le lieu privilégié par lequel s'établit le contact entre les espaces extérieurs et intérieurs, publics et domestiques (Zaccaria Ruggiu, 1995, p. 22-24 ; Battelli, 1998). C'est notamment le seul endroit de la maison que la plus grande partie des clientes a sans doute jamais vu, et dont certains n'ont d'ailleurs peut-être même jamais été admis à franchir le seuil. C'est donc là que s'affiche le plus visiblement le statut du propriétaire.

Très souvent précédée à l'extérieur d'un certain nombre de signes distinctifs, notamment d'une colonnade - qui, bien que donnant sur l'espace public, paraît avoir été à la charge des riverains -, l'entrée bénéficie du caractère public attaché à ce type d'aménagement urbain et s'en sert même quelquefois pour attirer l'attention sur elle par divers artifices; le plus courant étant, classiquement, de faire coïncider exactement l'entrée de la demeure avec un entrecolonnement. D'autres cas, plus sophistiqués, ne se sont pas contentés, pour concentrer l'attention sur elle, d'utiliser la colonnade simple, mais ont eu recours à des colonnes plus élevées, comme dans le cas, peut-être, de la Maison des Nones de Mars à Limoges (Loustaud, 2000, fig. 35). Dans la Maison au Grand Péristyle à Vieux (fig. 7), on a allié l'intégration à la colonnade publique d'une arche double (faisant visiblement référence à une porte de ville symbolique) et la focalisation du regard des passants sur celle-ci, par sa position dans l'axe d'un petit cardo débouchant en face de la maison, pour renforcer sa monumentalité (Vipard, 2001, p. 22-23, fig. 4-5).

L'atrium de la maison italique, en net déclin lors de l'implantation de la maison romaine en Gaule, semble cependant avoir eu une influence non négligeable sur la morphologie de la pièce d'accès.

52. Les corridors, fréquents dans les maisons bretonnes, semblent constituer une forme abâtardie de portique où l'aspect pratique l'emporterait sur l'aspect ostentatoire.
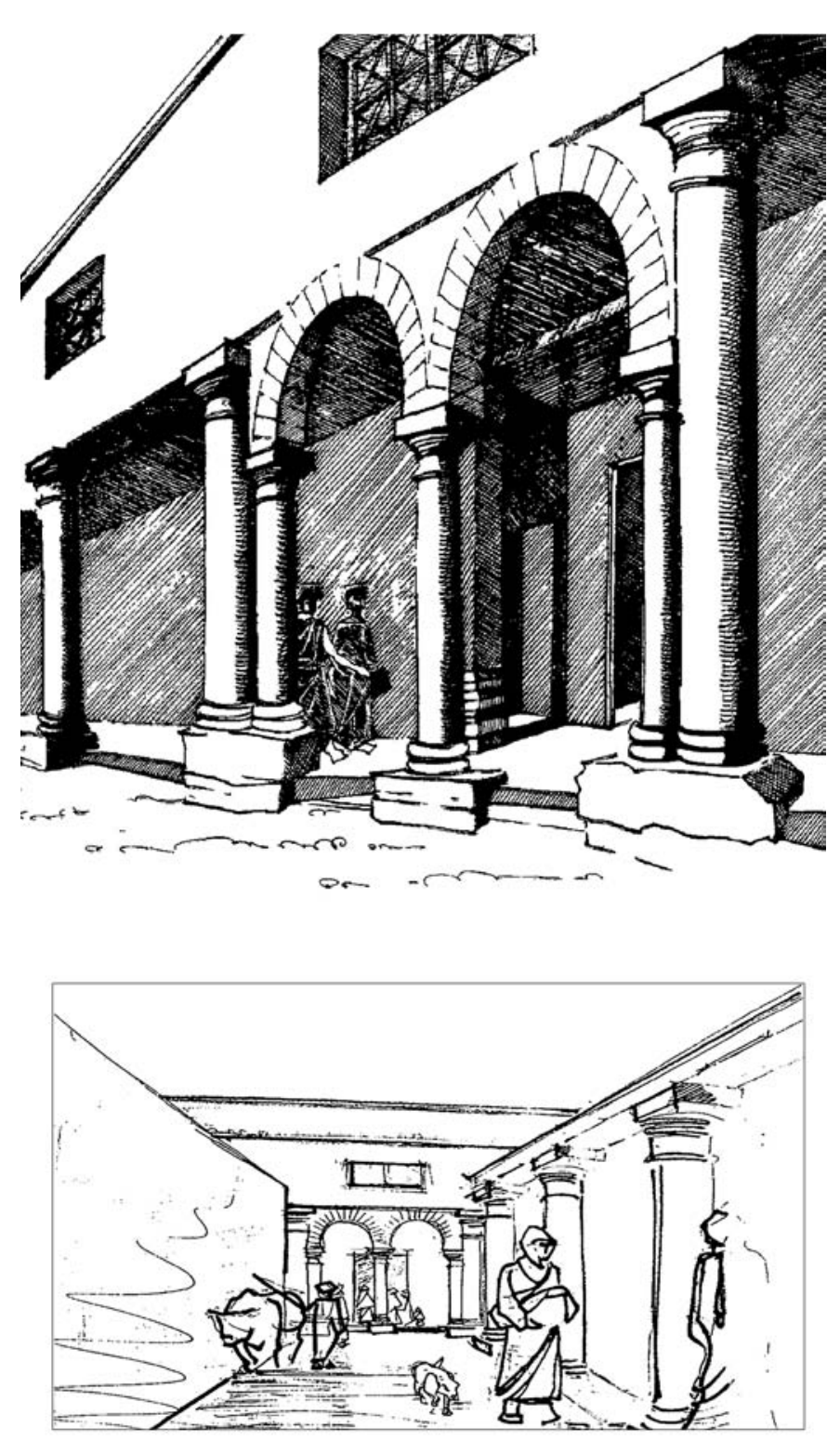

Fig. 7 - La Maison au Grand Péristyle à Vieux (Calvados) : un exemple de mise en scène de l'entrée et de l'utilisation de la topographie, début du III ${ }^{e}$ s. (d'après Vipard, 2001, fig. 4 et 5, p. 23).

Très nettement amorcée dès le milieu du $\mathrm{I}^{\mathrm{er}} \mathrm{s}$. av. J.-C., sous l'effet du report des activités qui y avaient lieu vers le péristyle, la disparition progressive de l'atrium en Italie même s'étale cependant jusque vers le milieu du $\mathrm{I}^{\mathrm{er}} \mathrm{s}$. apr. J.-C., voire un peu plus tôt (Dwyer, 1994, fig. 1, $\mathrm{n}^{\text {os }} 1-8$ ). Il n'est donc pas étonnant que l'on en trouve des traces en Gaule : en Narbonnaise, dès avant l'époque augustéenne, dans la Maison des Antes de Glanum (début du I ${ }^{\mathrm{er}}$ s. av. J.-C.), dans le premier état de la Maison au Dauphin de Vaison-laRomaine, au Clos de la Lombarde à Narbonne, dans les 


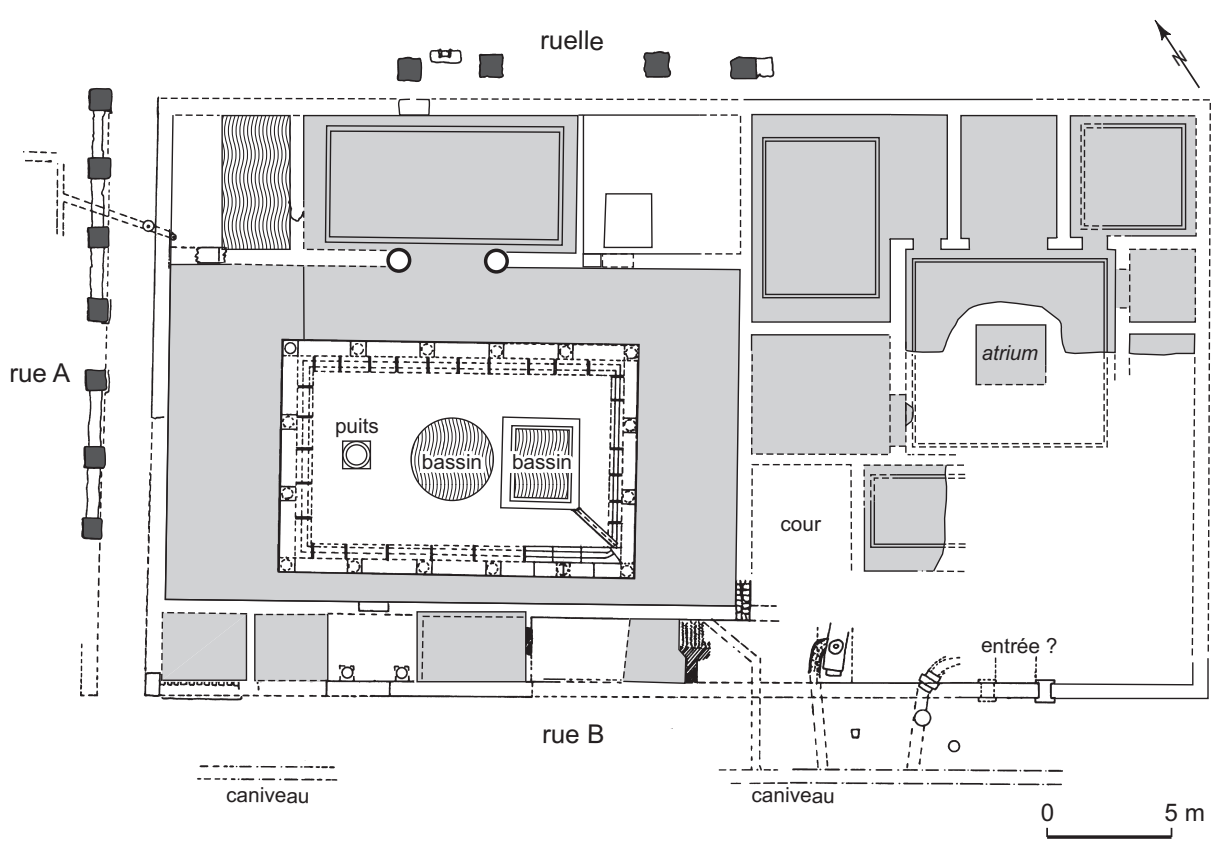

Fig. 8 - La Maison du Clos de la Lombarde à Narbonne (Aude) : état 1, vers 30 av. J.-C. (d'après Sabrié et al., 1987, fig. 25).

années 30-20 av. J.-C. ; un peu plus tard, à Saint-Raphaël, près de Fréjus (Maison du Suveret, première moitié du I ${ }^{\mathrm{er}} \mathrm{s}$. apr. J.-C.) (Jacob et al., 1990, p. 234), dans les Maisons viennoises du site des Nymphéas ( ${ }^{\mathrm{er}} \mathrm{s}$.) et de l'Atrium (milieu du I $^{\mathrm{er}}$ s.) (Collectif, 1996b, p. 416-420). Les agglomérations septentrionales ne sont pas en retard: au moins six maisons d'époque augustéenne précoce de Bibracte en sont ainsi dotées (Paunier, Luginbühl dir., 2004, p. 91-92), tout comme plusieurs maisons lyonnaises (Maisons du Laraire et des « Hauts de Saint-Just », voir Desbat et al., 1994, p. 195 et note 16) ; même la Germanie est concernée (Maison à Atrium de la cathédrale de Cologne, deuxième quart ou milieu du I ${ }^{\mathrm{er}}$ s., voir Fremersdorf, 1956).

En raison de l'obsolescence déjà avancée de cette forme architecturale à la fin de la République et surtout au début de l'Empire, on pourrait se demander si sa présence ou son absence dans une demeure pourrait être un signe de l'origine italienne ou indigène du propriétaire; mais il est possible, surtout au début de l'importation de modèles italiques, que l'on se soit contenté de transcrire fidèlement un plan sans en comprendre toute la signification et en l'utilisant pour se conformer à un mode de vie nouveau (Gros, 2001, p. 150-155). On doit toutefois constater que tous les modèles connus en Gaule semblent appartenir au modèle le plus tardif de l'évolution de cette forme en Italie; ils présentent en effet la particularité de ne pas recueillir l'eau de pluie, mais au contraire de l'évacuer, et de voir leur rôle désormais réduit à celui de puits de lumière. Cette dernière fonction pourrait d'ailleurs avoir également disparu et, de ce fait, l'importance numérique des atria tardifs pourrait être légèrement sous-estimée si l'on prend en considération l'existence probable de la forme d'atrium dite testudinatum où l'absence de colonnes rend difficile l'identification de la nature de la pièce.

Toutefois, même après l'abandon de cette forme dans les nouvelles constructions en Italie, on constate, en Gaule, des attitudes variables selon les propriétaires. Plusieurs semblent avoir volontairement conservé cet espace, sans doute pour des raisons de prestige qui s'attachaient aux formes anciennes, en complet accord avec ce mélange d'innovation et de conservatisme typique de l'esprit romain et apparemment bien assimilé par les élites gauloises. La conservation de l'atrium peut être le fait d'une absence de transformation d'une maison, dans le cas de la maison narbonnaise du Clos de la Lombarde (fig. 8), où il est utilisé jusqu'à l'abandon de la maison au $\mathrm{II}^{\mathrm{e}} \mathrm{s} .{ }^{53}$, par exemple, mais elle peut intervenir, et c'est plus significatif, malgré de profonds travaux de restructuration, comme à Saint-

53. À Rome même, des atria (testudinata) apparaissent encore dans d'assez nombreuses domus à péristyle d'époque sévérienne figurées sur la Forma Urbis (par exemple, Rodrígez Almeida, 1981, plans VIII, IX, XXXVII, XLVII, XLIX, LI, LII, LIX). 
Romain-en-Gal dans la Maison à Atrium, pendant le dernier quart du II ${ }^{\mathrm{e}}$ s. (Collectif, 1996b, p. 416-420). Dans ce cas, la volonté conservatrice est indéniable ${ }^{54}$. C'est donc peut-être plus à cause d'un attachement envers cette forme architecturale tout à fait obsolète et dénuée de ses fonctions originelles, mais à très forte valeur traditionnelle (même, et surtout, pour des Romains d'assez fraîche date) que par méconnaissance des modes architecturales (Gros, 2001, p. 157-158), que l'on pourrait expliquer des cas de création tardifs (et donc volontairement archaïsants). Le plus net est celui de la Maison au Dauphin, à Vaison-la-Romaine, qui apparaît à l'état 4, c'est-à-dire seulement à la fin du $\mathrm{II}^{\mathrm{e}} \mathrm{s}$. ou dans la première moitié $\mathrm{du} \mathrm{III}^{\mathrm{e}} \mathrm{s}$., par transformation du vestibule en atrium tétrastyle avec un impluvium (évacuant l'eau vers l'égout) et qui s'accompagne d'ailleurs de la création d'un tablinum le mettant en communication avec le péristyle.

L'atrium ne constitue toutefois pas le modèle le plus fréquent d'espace d'entrée des maisons en Gaule, même si des réminiscences, souvent négligées, se perpétuent dans les entrées des maisons d'âge impérial. Celles-ci présentent généralement des vestibules simples assurant la transition directe entre la rue et le péristyle.

Cet espace d'entrée n'est jamais inexistant. Sa forme est variable, mais généralement simple, et se réduit à trois types essentiels dont les deux premiers, rares en Gaule, se rencontrent essentiellement dans des maisons plutôt modestes et peu étendues : le premier est constitué d'un minuscule sas (cas de la Maison au Bassin Excentré à Saint-Romain-enGal) ; le deuxième se présente sous la forme d'un couloir étroit, comme dans la Maison de Sucellus à Saint-Romainen-Gal ou dans celle du Génie Privé à Martigny, dont le rôle paraît essentiellement utilitaire et sans doute destiné à isoler le cœur de la maison de l'extérieur (et notamment du bruit), peu approprié à la réception éventuelle de clientes. Dans tous ces cas, on peut alors supposer que le péristyle n'a qu'un rôle d'agrément et que l'on se trouve en présence de notables modestes pour qui il n'est pas nécessaire (ou possible) de sacrifier trop de place à cet espace d'entrée.

Le troisième cas de figure, de loin le plus répandu, est constitué d'une salle, plus souvent rectangulaire que carrée, de dimensions très variables, généralement assez vaste et simple, mais qui peut quelquefois présenter divers degrés de complexité architecturale. La porte est le plus souvent située sur le même plan que le mur de façade.

54. On pense alors, dans un cas comme dans l'autre, à l'affection que manifeste Pline le Jeune, à la toute fin du I ${ }^{\mathrm{er}} \mathrm{s}$. apr. J.-C., pour l'atrium ex more veterum ("à l'ancienne») de sa villa de Toscane (Epistulae, V, $6,15)$.
Contrairement aux cas précédents, l'ampleur, la monumentalité affirmée et, pour autant qu'on puisse le savoir, la décoration luxueuse de cet espace charnière entre les domaines privé et public, laissent plutôt supposer un rôle d'accueil d'un certain nombre de personnes étrangères à la maisonnée.

Une fois encore, on a souvent cherché à expliquer l'origine de cette forme d'entrée de la maison romaine par une influence grecque (voir notamment, Étienne, 1960, p. 118-120). Celle-ci ne peut être totalement exclue dans quelques cas, mais les indices que l'on possède semblent faire apparaître que les vestibules élargis de Gaule sont plutôt le résultat d'une évolution à partir d'éléments italiques dont ils ont absorbé l'espace et une partie des fonctions. À partir de l'enfilade républicaine classique «vestibulum ${ }^{55}$-fauces, atrium, tablinum, péristyle ", on aurait abouti, après disparition de l'atrium et du tablinum, à un schéma toujours axial : «porche éventuel (vestibulum), vestibule (fauces et espace de l'atrium, voire du tablinum), péristyle » ${ }^{56}$. L'étape «porche éventuel, vestibule, tablinum, péristyle »n'a peut-être pas été systématique, quand le tablinum a disparu en même temps que l'atrium, mais son existence est néanmoins certaine dans la Maison des Nones de Mars à Limoges (fig. 9) où l'on rencontre la succession du porche 37 , du vestibule 30 , du tablinum 25 et du portique $16^{57}$. Cette étape semble avoir eu un certain succès puisqu'on en retrouve des séquences voisines dans les cas de recréation tardifs de la Maison au Dauphin à Vaison-la-Romaine (état 4, début du $\mathrm{III}^{\mathrm{e}} \mathrm{s}$.) et de celle au Petit Péristyle à Vieux (fin du $\mathrm{II}^{\mathrm{e}} \mathrm{s}$.). La Maison au Grand Péristyle qui succède à cette dernière aux alentours de 200, voit la disparition du «tablinum», mais la création d'une entrée de type vestibulum-fauces dont la séparation est marquée par une cloison dans laquelle s'insérait une porte en chêne à deux battants, caractéristiques des entrées nobles. Le cas n'est pas isolé dans les vestibules gaulois. Cette bipartition s'accompagne souvent de l'existence d'un changement d'altitude. D'ultimes traces de cette bipartition paraissent avoir subsisté dans certains cas de vestibules simples (dont la porte était au niveau du mur de façade, du

55. Sur ce court espace (sorte de porche) appartenant au monde extérieur, mais pénétrant néanmoins dans la maison, et séparé de l'espace intérieur (les fauces) par la porte, voir notamment Lafon, 1995.

56. Cas de la Maison à l'Opus Quadratum de Limoges (seconde moitié $\mathrm{du} \mathrm{I}{ }^{\mathrm{er}} \mathrm{s}$.), dont le vestibule possède d'ailleurs un bassin rappelant un impluvium.

57. Les deux petites exèdres du vestibule 30 semblent d'ailleurs également conserver le souvenir des alae ouvertes cantonnant les atria traditionnels (Loustaud, 1992, p. 79). 


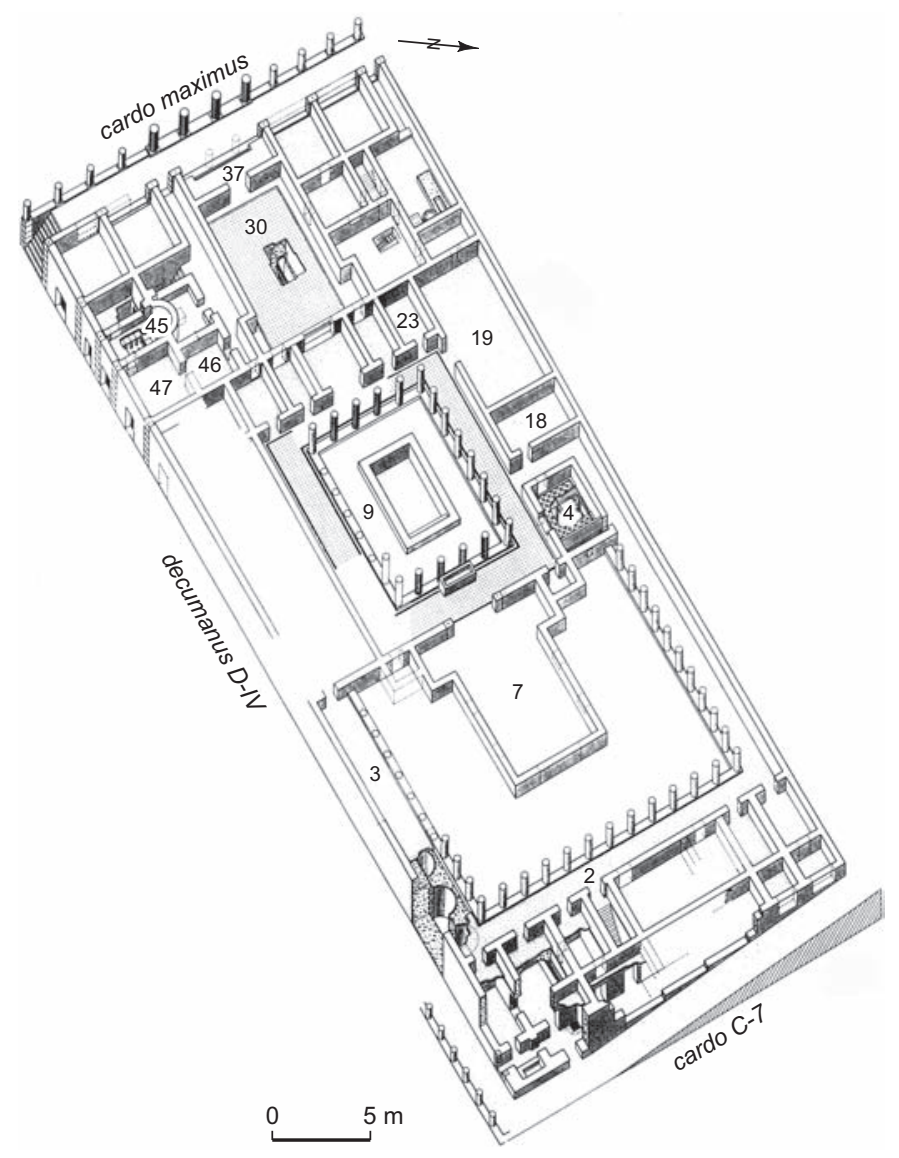

Fig. 9 - Plan axonométrique restitué de la Maison des Nones de Mars à Limoges (Haute-Vienne), vers 30-40 apr. J.-C. (d'après Loustaud, 2000, fig. 30).

moins on le suppose), par exemple sous la forme du changement de niveau marqué par deux marches dans la Maison du Chantier Delbès, à Saint-Bertrand-de-Comminges (May, 1986, p. 124-125), voire sous celle d'une simple rupture dans le décor du pavement du vestibule mosaïqué de la Maison du Dieu Marin à Aix-en-Provence (Collectif, 1996b, p. 44-45).

Que les vestibules aient hérité d'une partie de l'espace jadis occupée par l'atrium ressort également de l'existence de colonnes ou de piliers supportant un toit fermé et de l'existence d'un bassin central, permettant de conserver (ou de redonner) à la pièce un caractère ancien. C'est par exemple le cas, à Saint-Romain-en-Gal, de l'entrée de la Maison aux Pierres Dorées (vers 15-20 apr. J.-C.) qui semble avoir été d'abord un vestibule couvert plutôt qu'un atrium testudinatum, assurément couvert à l'état 3 (Maison du Vestibule à Colonnes, vers 50-60), mais alors doté de huit piliers et d'un bassin lui conférant un aspect d'atrium. Vers 160-180, dans la Maison des Dieux Océan qui lui succède, un bassin circulaire perpétue le souvenir de l'atrium et de son impluvium, malgré la suppression des colonnes. Un tel cas montre comment la forme, même disparue, continue à marquer l'architecture du lieu.

$\mathrm{Si}$, comme on peut s'en douter, le vestibule élargi des maisons de Gaule (et d'autres provinces) dérive de l'atrium républicain, il n'en a pas conservé les fonctions triviales (collecte de l'eau et éclairage), mais seulement les fonctions nobles (réception). L'importance des dimensions de cet espace trouverait donc une justification dans les besoins d'ostentation et de réception de la clientèle.

Hormis les cas évoqués dans la discussion sur l'évolution de l'atrium, les vestibules ne présentent apparemment pas beaucoup d'aménagements structurels notables et, sorti des critères morphologiques simples (forme barlongue ou longue et étroite, voire atypique), on dispose de peu d'éléments pour essayer d'apprécier, à partir des entrées, ce qu'a pu être le rang d'une domus. Sans doute moins sûrement que le luxe, malheureusement non mesurable, les dimensions des espaces d'entrée restent néanmoins trop souvent le seul élément utilisable (tabl. II).

La taille de l'entrée représente entre $1,5 \%$ à $6 \%$ de la domus. L'utilisation des proportions de cet élément par rapport à la maison elle-même ou au péristyle montre que, contrairement à celle du péristyle, la croissance de la surface de l'entrée par rapport à celle de la maison n'est pas proportionnelle, ou, du moins, pas aussi élevée. Indépendamment de la taille réelle de la domus, l'obtention d'une taille jugée satisfaisante ou adaptée aux besoins attendus pourrait être une raison de la faible part occupée par cet espace dans les très vastes maisons. Notre méconnaissance des pratiques et des aspects matériels de la réception clientélaire dans les provinces sous l'Empire ne permet pas de chercher de réponses assurées dans cette direction. On ne peut que supposer qu'elle a pu jouer un rôle dans la dimension de cette pièce. En s'en tenant au seul critère de taille, les entrées des Maisons à Atrium de la cathédrale à Cologne, des Dieux Océan à Vienne ou des Nones de Mars à Limoges, auraient été alors susceptibles d'accueillir plus de 500 personnes ${ }^{58}$; même les cas plus modestes descendraient alors rarement en dessous d'une centaine de personnes. La faible amplitude de variation de taille entre des maisons fort différentes ou le fait que des maisons relativement modestes aient quelquefois une entrée supérieure à celle de grandes domus montrent

58. Respectivement environ 525, 890 et 1145 si l'on prend pour base l'estimation de 5,8 personnes au mètre carré utilisée pour restituer le nombre de clientes pouvant tenir dans l'atrium de M. Aemilius Scaurus, à Rome (Coarelli, 1989, p. 182). 
Tabl. II - Dimensions de quelques salles de réception gauloises et bretonnes : 1, taille de la maison en mètres carrés; 2, dimensions (approximatives) de l'entrée ; 3, surface en mètres carrés ; 4, pourcentage par rapport à la surface de la maison.

\begin{tabular}{|c|c|c|c|c|c|}
\hline Localisation & Dénomination & 1 & 2 & 3 & 4 \\
\hline \multicolumn{6}{|l|}{ Maisons de plus de $2000 \mathrm{~m}^{2}$} \\
\hline Limoges (Haute-Vienne) & Maison à I'Opus Quadratum & 2300 & $12 \times 12$ & 144 & 3,1 \\
\hline Saint-Romain-en-Gal (Rhône) & Maison des Dieux Océan, état 4, salle 12 & 2932 & $9 \times 14,5$ & 130,5 & 4,4 \\
\hline Limoges (Haute-Vienne) & Maison des Nones de Mars & 3735 & $9 \times 11(+$ alae $)$ & 115 & 3,1 \\
\hline Saint-Romain-en-Gal (Rhône) & Maison sous le Lycée & $5000 \mathrm{~min}$. & $10 \times 11$ & 110 & 2,2 \\
\hline Limoges (Haute-Vienne) & Maison à l'Emblema au Lion & 2300 & $12,6 \times 7,80$ & 98,3 & 4,3 \\
\hline Saint-Romain-en-Gal (Rhône) & Maison au Lion & 3100 env. & $9 \times 10$ & 90 & 2,9 \\
\hline Périgueux (Dordogne) & Maison de la rue des Bouquets & $2350 \mathrm{~min}$. & $8,5 \times 7,5$ & 64 & 2,7 \\
\hline Saint-Romain-en-Gal (Rhône) & Maison des Dieux Océan, état 3, salle 14 & 2932 & $6 \times 7,5$ & 45 & 1,5 \\
\hline Vaison-la-Romaine (Vaucluse) & Maison au Dauphin & 2700 & $4,5 \times 8$ & 36 & 1,3 \\
\hline \multicolumn{6}{|l|}{ Maisons de 1000 à $2000 \mathrm{~m}^{2}$} \\
\hline Caerwent (Grande-Bretagne) & Maison de I'Insula IIIS & 1980 & $9 \times 11,5$ & 103,5 & 5,2 \\
\hline Vieux (Calvados) & Maison du « Bas de Vieux », salle 3 & 1421 & $7,80 \times 9,20$ & 72 & 5,1 \\
\hline Metz (Moselle) & Maison de l'Arsenal & 1350 env. & $6,75 \times 8,75$ & 59 & 4,4 \\
\hline Alba-la-Romaine (Ardèche) & Maison du Champ Delauzun & $1420 \mathrm{~min}$. & $6 \times 8,5$ & 51 & 3,6 \\
\hline Saint-Romain-en-Gal (Rhône) & Maison des Dieux Océan, état 2 , salle T & 1326 & $6,5 \times 6,5$ & 42 & 3,2 \\
\hline \multicolumn{6}{|l|}{ Maisons de $1000 \mathrm{~m}^{2}$ ou moins } \\
\hline Lyon (Rhône) & Maison aux Masques & 800 env. & $6,5 \times 10,5$ & 68,5 & 8,6 \\
\hline Glanum (Bouches-du-Rhône) & Maison VI des Antes, état 3 & 500 & $7,5 \times 7,5$ env & 60 & 12 \\
\hline Saint-Romain-en-Gal (Rhône) & Maison aux Cinq Mosaïques & 500 & $7,5 \times 7,5$ env & 60 & 12 \\
\hline Orange (Vaucluse) & Maison à la Mosaïque Polychrome & 900 & $6 \times 9,5$ & 57 & 6,3 \\
\hline Narbonne (Aude) & Maison du Clos de la Lombarde & 975 & $5 \times 11$ & 55 & 5,6 \\
\hline Lyon (Rhône) & Maison aux Xenia & 660 env. & $5,5 \times 9$ & 49,5 & 7,5 \\
\hline Fréjus (Var) & Maison de la place Formigé, état 1 & 462 & $5,5 \times 5,5$ & 30,25 & 6,5 \\
\hline Glanum (Bouches-du-Rhône) & Maison VIII d'Atys & 480 & $5 \times 4,5$ & 22,5 & 4,7 \\
\hline
\end{tabular}

qu'il serait hasardeux de supposer un ratio déterminé entre la taille de la pièce et celle de la clientèle (donc, de la puissance) d'un patronus. Outre le fait que tous les clientes - destinataires et vecteurs privilégiés de l'ostentation, sous l'Empire - n'entraient pas physiquement dans la maison, la puissance d'un patronus ne s'établissait pas uniquement sur des critères quantitatifs, mais sans doute également sur son aptitude à manifester sa puissance à travers la richesse du décor et la mise en scène de celui-ci dont on perçoit très vaguement l'importance à travers les bribes conservées : piliers sculptés, bassins, mosaïques ou peintures. En fonction de ce que l'on connaît par ailleurs, il est en effet plus que probable que certains vestibules aient compensé leurs dimensions moyennes par la majesté de leur décor.

Quoi qu'il en soit de toutes ces incertitudes, ce que l'on entrevoit de l'entrée de la maison et de son rôle privilégié aux confins entre les sphères publique et privée montre son importance au sein de la trilogie de ses composantes publiques.

\section{Les salles de réception}

Le dernier élément-clé de la trilogie est plus complexe à étudier que les deux autres parce qu'il comprend en fait des pièces que la fouille ne permet pas toujours d'identifier clairement. Il correspond à une, souvent deux (voire plusieurs) grandes salles de réception se distinguant nettement des autres par leurs dimensions, leur luxe et leur situation privilégiée par rapport au péristyle.

Morphologiquement, ces salles sont moins spécialisées que dans la théorie vitruvienne. Cela est dû à l'évolution de la société de l'époque impériale, notamment à une modifi- 
cation des modes d'expression de la richesse, parallèlement à la diminution ou à l'arrêt des luttes électorales pour accéder au pouvoir, sous l'effet du renouvellement progressif des élites urbaines entre le milieu du $\mathrm{I}^{\mathrm{er}} \mathrm{s}$. et la fin du $\mathrm{II}^{\mathrm{e}}$ s., et à une perte d'attrait de la fonction décurionale.

Ces salles résultent en effet de la fusion d'un certain nombre d'espaces et ont notamment absorbé les fonctions autrefois dévolues au tablinum ou aux basiliques privées ${ }^{59}$. Elles peuvent être qualifiées de salles de réception (terme qui ne préjuge en rien d'une activité spécifique) et peut-être même plus précisément, étant donné leur position et leur luxe et si l'on s'en tient à leur usage le plus évident, de salles d'apparat ${ }^{60}$. Leur utilisation exacte n'est pas déterminable avec précision : réception d'hôtes variés, d'amis ou de clients pour le traitement des affaires, chambre temporaire pour un invité ou sa suite, etc. Il ne fait toutefois guère de doute qu'elles aient également servi de salles à manger, sans pour autant nécessairement avoir été seulement des triclinia ${ }^{61}$.

Quelle que soit l'appellation qu'on leur donne, ces pièces présentent des caractéristiques qui affirment leur position dominante et les associent d'emblée aux deux premiers éléments de la trilogie: leur grande taille, leur ouverture large, leur sol fréquemment situé à une altitude plus élevée que les autres, leur articulation privilégiée avec le péristyle (notamment par la recherche d'un point de vue sur les aménagements les plus marquants du jardin, favorisé par la coïncidence de leur entrée avec un entrecolonnement) ; surtout, un décor luxueux - généralement le plus riche de la maison - mais conventionnel, seyant à leur dignité. Ces pièces sont en outre apparemment souvent dotées de hauts plafonds stuqués et/ou voûtés et leurs sols, quand il y a des mosaïques dans la demeure, sont les premiers, et souvent les seuls, à en être ornés.

59. Ce terme ne correspond pas à une forme (salle à quadriportique), mais à une fonction: lieu de réunion où se prennent les décisions intéressant la communauté et où se règlent les problèmes entre le patron et ses dépendants. Sur cette pièce, voir l'étude très pertinente de P. Gros, 2004.

60. On trouve souvent employées les appellations exus (sans doute pas toujours doté de colonnes intérieures, contrairement à ce laisserait penser une lecture rapide de Vitruve, De Architectura, VI, 3, 8-10) ou exedra (salle complètement ouverte sur l'un de ses côtés), qui désignent des formes architecturales susceptibles d'avoir abrité des fonctions identiques et différant peut-être surtout par la nature de leur ouverture. L'état d'arasement des murs ou les plans disponibles ne permettent pas toujours de déterminer la nature de celle-ci.

61 . Il ne faut pas perdre de vue que ces salles à manger sont clairement rangées par Vitruve (De Architectura, VI, 5, 1) dans la catégorie des pièces privées, mais la richesse et la décoration de certaines laissent supposer, sous l'Empire, un usage ostentatoire et donc leur appartenance aux parties publiques de la maison.
En l'état de la documentation archéologique, les salles conservées présentent le plus souvent la forme d'un rectangle assez trapu, qui rend d'autant plus impressionnantes des formes plus complexes comme le grand salon 7 de la Maison des Nones de Mars visiblement conçu pour fonctionner avec le grand jardin postérieur sur lequel il avait vue à travers les longs côtés de la pièce (ceus cyzicène de Vitruve, VI, 3, 11). On notera au passage qu'en Gaule on ne trouve que très peu de ces plans à absides dont le rôle judiciaire est bien attesté au $\mathrm{I}^{\mathrm{er}} \mathrm{s}$. av. J.-C. (basiliques privées) et si typique du Bas-Empire (pour la réception de ses dépendants par le patronus) ${ }^{62}$.

L'étude des dimensions de ces pièces montre que, comme pour les autres éléments-clés, les plus grandes salles de réception se rencontrent dans les plus grandes domus, mais que le rapport entre la taille des deux éléments n'est pas constant. En valeur absolue, de petites maisons ont quelquefois des salles de réception égales ou supérieures à celles des plus grandes ${ }^{63}$. L'examen des surfaces, mais également celui du pourcentage de la surface occupée par la grande salle de réception dans la superficie de la domus: environ $1,5 \%$ à 4,5\% des grandes domus (plus de $2000 \mathrm{~m}^{2}$ ), $3 \%$ à $7 \%$ des moyennes (entre 1000 et $2000 \mathrm{~m}^{2}$ ), et $5 \%$ à $10 \%$ ou $12 \%$ des petites (moins de $1000 \mathrm{~m}^{2}$ ), fait clairement apparaître qu'il ne semble pas y avoir eu, comme pour le péristyle, de recherche d'agrandissement démesurée. D'un autre côté, un seuil minimal semble avoir existé (d'où un pourcentage de superficie élevé dans les plus petites maisons). Ces éléments font bien ressortir le caractère indispensable de cette pièce, et le fait qu'une surface de $50 \mathrm{~m}^{2}$ à $100 \mathrm{~m}^{2}$ suffisait à satisfaire les besoins de la majorité des maisons riches de Gaule.

\section{Organisation et structure de l'espace}

L'importance des trois espaces publics (péristyle, entrée, salles de réception) ressort de la superficie importante qu'ils occupent par rapport à la surface totale de la domus et aux espaces purement résidentiels ou de service. Elle est

62. Quelques salles présentant cette disposition se rencontrent toutefois épisodiquement sous le Haut-Empire, comme celle - d'usage indéterminé - de la Maison au Grand Péristyle ( ${ }^{\mathrm{er}} \mathrm{s}$. ?) à Aix-en-Provence (Collectif, 1996b, p. 31). Une salle de réception à abside à décor de scènes mythologiques est créée dans la Maison de l'Insula XIII Est à Avenches (Suisse) dans la seconde moitié du $\mathrm{II}^{\mathrm{e}} \mathrm{s}$. (Paunier, 1996, p. 104) et, lors des transformations postérieures à 226-235, une abside et un opus sectile sont ajoutés au grand salon VI de la Maison à l'Opus Quadratum à Limoges (Loustaud, 2000, p. 199-200).

63. Mais plusieurs salles de réception existaient généralement dans ces dernières. 


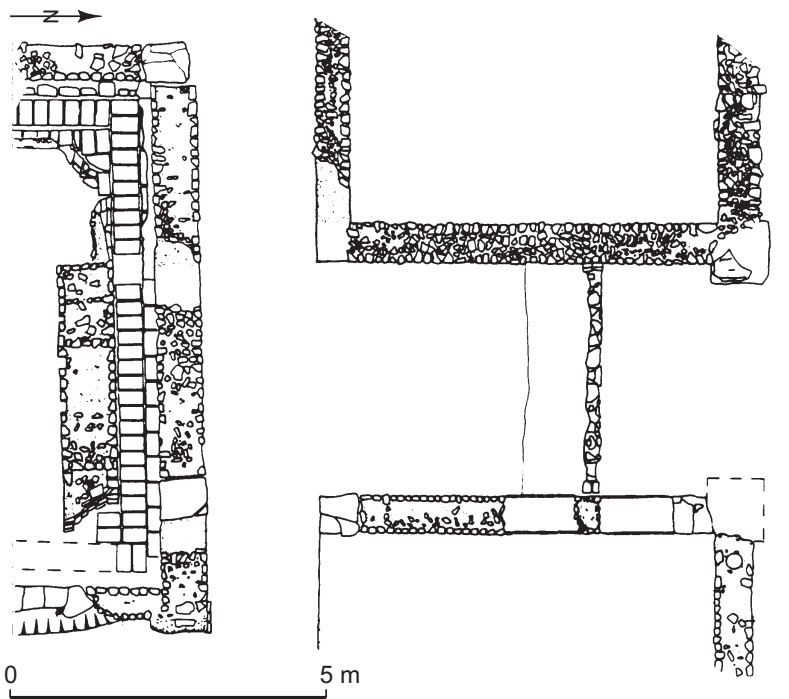

1

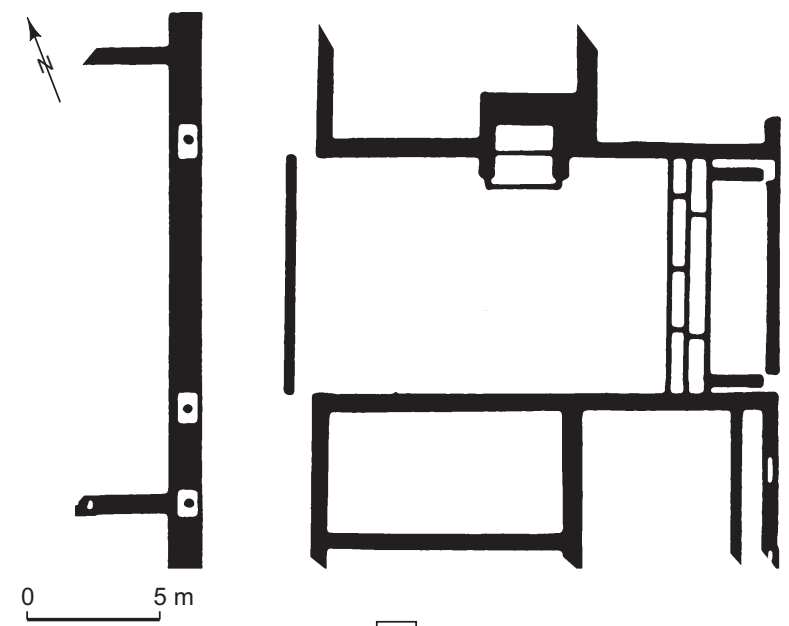

2

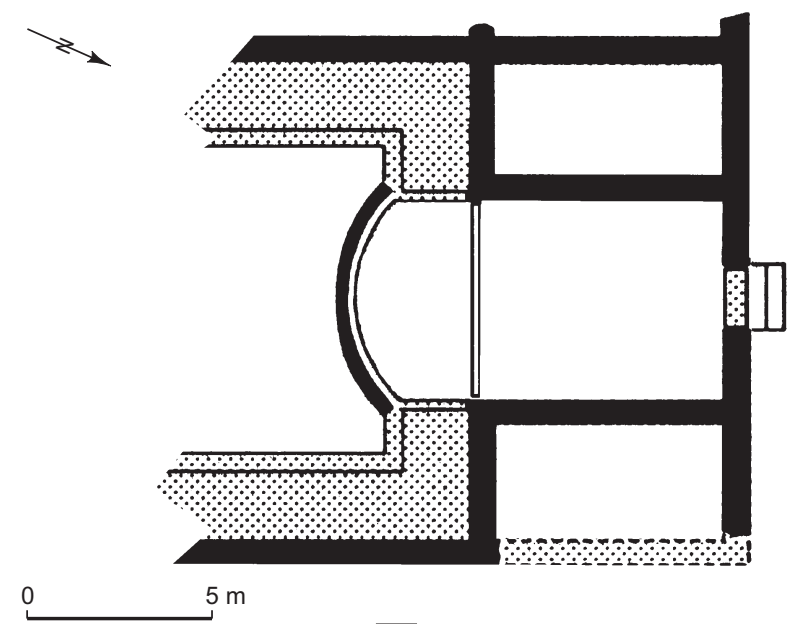

3
Fig. 10 - Trois entrées présentant un aménagement empêchant la vue sur le péristyle : 1, Maison au Grand Péristyle à Vieux (Calvados), vers 200 apr. J.-C. (d'après Vipard, 1996) ; 2, Maison du Chantier Delbès à Saint-Bertrand-de-Comminges (Haute-Garonne) (d'après May, 1986, p. 124) ; 3, Maison à la Salle en Hémicycle à Aix-en-Provence (Bouches-du-Rhône), seconde moitié du I I ${ }^{e r}$. (d'après Collectif, 1996b, p. 41).

également mise en évidence par le fait qu'ils sont, de loin, les trois lieux les plus décorés de la maison, mais surtout par leur liaison organique et leur disposition privilégiée les uns par rapport aux autres.

L'axialité, à juste titre considérée comme l'un des signes majeurs de l'esprit architectural romain, a visiblement été recherchée et les cas où entrée, péristyle et salle de réception principale sont alignés, sont effectivement nombreux (Meyer, 1999), mais on y a apparemment assez souvent renoncé au profit de la perpendicularité en cas de contraintes. Ce point semble faire ressortir que, plus que l'axialité des éléments de la trilogie, c'est l'organisation de l'entrée et des salles de réception autour du pivot constitué par le péristyle qui joue le rôle le plus important. Dans ce cas de figure, ou lorsque existe une seconde salle de réception importante, le choix de la position de celle-ci semble avoir été plus influencé par la place de l'entrée de la demeure à étudier que par des considérations cardinales. Hormis quelques exceptions, la salle de réception apparaît en effet presque toujours située à main droite de la personne venant du vestibule, pratique sans doute liée au caractère positif du côté droit.

La position de l'entrée ne semble pas avoir été un élément des plus contraignants. Même quand une rue ou un espace public dominants imposaient sa localisation sur le côté le plus large du bâtiment (donc le moins favorable à l'axialité interne), le bon fonctionnement de la maison n'en était pas remis en question. Même dans ce cas d'ailleurs, on constate qu'on a quelquefois réussi à conserver la disposition axiale en ayant recours à un péristyle plus large que profond afin de compenser l'insuffisance de profondeur de la maison 64 .

Que la disposition des éléments ait été axiale ou perpendiculaire ne changeait rien au cheminement logique entre eux. Dans les deux cas également, ce qui semble avoir différencié le vestibule élargi des salles de réception, c'est la liaison visuelle existant entre ces éléments et le péristyle :

64. Cette disposition barlongue du péristyle, qui n'est pas la plus fréquente, est pourtant celle préconisée par Vitruve (De Architectura, VI, 3, 7). 
dans le cas des salles de réception, elle a toujours été privilégiée, alors que dans le cas du vestibule, elle semble, sous l'Empire, avoir été assez souvent entravée par un certain nombre d'obstacles (fig. 10). Outre d'éventuels écrans plus ou moins mobiles (tentures ou paravents), comme on en connaît ailleurs, les séparations placées entre le vestibule et le péristyle sont généralement constituées par un décalage des ouvertures ou un véritable mur. Dans ce cas, leur présence est une de ces nombreuses barrières, physiques ou mentales, qui rythment pour le public la hiérarchie des autorisations d'accès au cœur de la maison. Elle montre également une certaine évolution de la perception du caractère public du péristyle, autrefois plus visible à défaut d'être accessible physiquement, et que l'axialité et la perspective qu'elle permet ne sont finalement pas (ou plus) des éléments déterminants dans leur disposition architecturale.

\section{La décoration et le luxe}

Il nous manque tant d'éléments de leur équipement et de leur parure intérieure d'origine qu'il est difficile d'établir une véritable hiérarchie entre des maisons dont les ruines nous paraissent assez semblables.

L'archéologie ne vient pas confirmer, ni même vraiment permettre de supposer, que les maisons gauloises regorgeaient de statues, à l'instar de Rome (Guidobaldi, 1986, p. 217) ou des villes de Campanie. Le peu que l'on possède semble avoir été essentiellement concentré dans les parties publiques et en particulier le péristyle, comme on a eu l'occasion de le voir, et encore faut-il faire remarquer que les deux seuls lots utilisables en Narbonnaise, celui du péristyle de la Maison des Dieux Océan à Saint-Romain-enGal et celui, nettement plus étoffé, provenant du vestibule, de l'atrium, du tablinum et du péristyle de la Maison au Buste d'Argent à Vaison-la-Romaine (respectivement Jockey, 1996, p. 203-205 et p. 209-210), ne sont peut-être finalement pas si représentatifs du simple décor domestique, puisque ces bâtiments pourraient bien être des sièges de collèges (Gros, 1997). D’autres traces, rares et d'autant plus précieuses, sont peut-être plus significatives. Ainsi la statue de Tutela retrouvée dans le plus beau salon d'apparat de la Maison au Grand Péristyle de Vieux (Vipard, 1992 et 2001, p. 29-30 et fig. 15) ou son homologue, connue seulement par son socle dédicacé, de la Maison de Balbius Iassus à Autun ${ }^{65}$

65. La main gauche tenant une patère, retrouvée dans la Maison des Dieux Océan, à Saint-Romain-en-Gal (Jockey, 1996, p. 205, fig. 4), pourrait avoir appartenu à une divinité comparable. Peut-être faut-

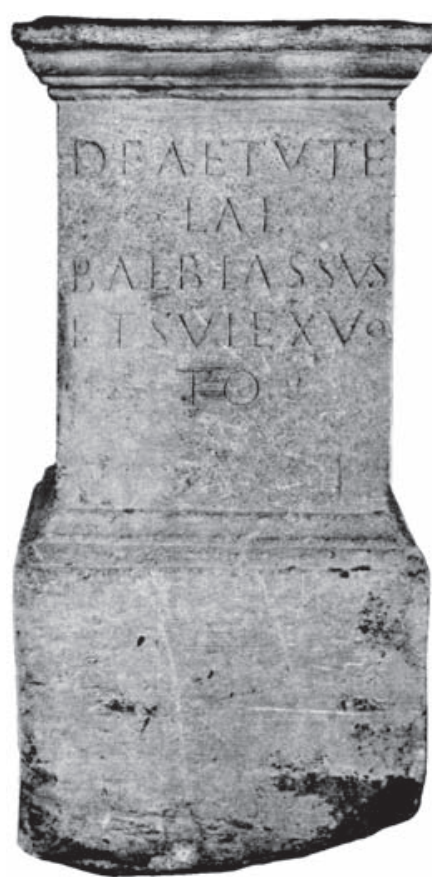

1

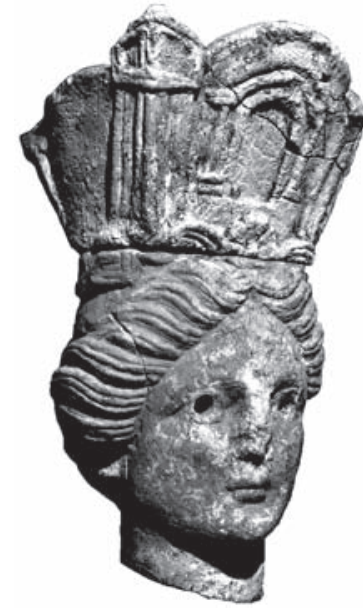

2
Fig. 11 - La déesse Tutela : 1, autel de la Maison de Balbius Iassus à Autun (Saône-et-Loire) (d'après Blanchard-Lemée et al., 1986, fig. 2 p. 123) ; 2, tête de la statue du salon II de la Maison au Grand Péristyle à Vieux (Calvados) (d'après Vipard, 2001, fig. 15).

(Blanchard-Lemée et al., 1986, p. 122, fig. 2) (fig. 11). Ce n'est sans doute pas un hasard si de telles œuvres, à forte connotation officielle, proviennent de maisons de notables et, dans un cas au moins, d'une pièce à caractère public très marqué.

Les éléments du décor architectonique, eux, ont généralement mieux subsisté du fait des difficultés posées par leur déménagement; l'un des plus connus est bien évidemment le décor pictural qui n'est pourtant pas le plus abondant ni le mieux conservé ${ }^{66}$.

Dans ce domaine, la Gaule se contente dès le début de suivre les grands courants picturaux. Comme l'a bien exposé Y. Perrin (1997), le décor domestique commençant sous Auguste, on a donc eu recours au III ${ }^{\mathrm{e}}$ style, répondant à la simplicité requise par l'idéologie impériale du moment,

il ajouter à ce lot la déesse-mère de la domus pétrucore de la rue des Bouquets, à Périgueux, dont A. Bouet rappelle qu'elle peut être assimilée à Fortuna, Abundantia ou Tutela (Bouet, 2001, p. 254).

66. Pour un aperçu de la question en Gaule, voir A. Barbet et al., 1996 (avec, notamment, p. 38-42, la liste sommaire des lots disponibles des principales maisons urbaines à décor peint). Sur la diffusion des styles, voir Barbet, 1987; pour la Narbonnaise en particulier, beaucoup d'éléments dans Sabrié et al., 1987. 


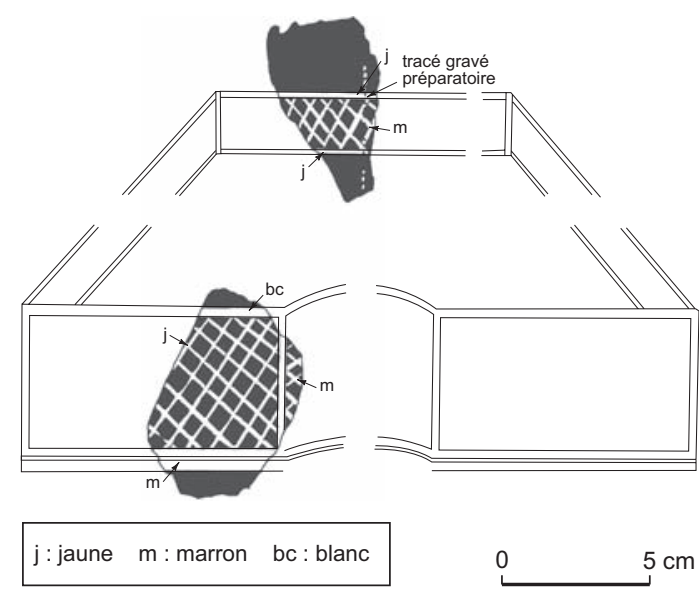

Fig. 12 - Décor de III style pompéien typique : hortus conclusus sur fond noir de la Maison de la rue des Bouquets à Périgueux (Dordogne), vers 45 apr. J.-C. (d'après Barbet et al., 2004, fig. 2, p. 154).

mais concurremment au $\mathrm{II}^{\mathrm{e}}$ style, décor architectonique plus sophistiqué élaboré dans les dernières décennies de la République pour exprimer les besoins politiques et culturels de l'élite. Ce dernier style, sans doute apprécié pour sa référence à des traditions anciennes et romaines et sa convenance, se conserve plus longtemps qu'en Italie, même en Narbonnaise (et particulièrement à Narbonne, voir Sabrié et al., 1987, p. 186) et le III ${ }^{\mathrm{e}}$ style à panneaux, assez unitaire sous Auguste, plus diversifié sous Tibère et Caligula, perdure également ainsi quelquefois sous la forme du système à candélabres dans le IV ${ }^{\mathrm{e}}$ style ${ }^{67}$ qui triomphe à partir de Claude (par exemple à Aix-en-Provence, Vienne, Orange, Nîmes et Narbonne). Le IV style, éclectique, est utilisé partout au $\mathrm{II}^{\mathrm{e}}$ s., et jusqu'à l'époque des Sévères ; on y voit apparaître tardivement le recours à quelques mégalographies et le retour à des architectures grandioses, constituant en quelque sorte un chant du cygne, en milieu urbain gaulois puisque les programmes décoratifs cessent, après le début $\mathrm{du} \operatorname{III}^{\mathrm{e}} \mathrm{s}$, , avec la disparition accrue des maisons urbaines riches.

À de rares exceptions près ${ }^{68}$, les stucs dont l'emploi est généralement subordonné au décor peint, notamment aux motifs de séparation et aux plafonds, n'ont pas laissé de traces remarquables (Frizot, 1977).

67. Sur ce style et ses formes variées, voir Barbet, 1980.

68. Comme un personnage à bonnet phrygien (sans doute un Attis), en haut-relief et en demi-grandeur nature, de la salle XV de la domus du «Bas de Vieux ", installé dans un édicule peint en trompe-l'œil (Vipard, 1998, p. 87).
L'impression d'une différence entre la Narbonnaise et les Trois Gaules, qui pouvait être supposée jusqu'à une date récente, est en train de se gommer et semble en fait tenir plutôt au décalage chronologique des programmes de décoration et au conservatisme culturel ${ }^{69}$. Une preuve éclatante en a été administrée par le superbe décor, d'aspect tout campanien, de la Maison des Nones de Mars à Limoges, selon toute vraisemblance réalisé dans les années 35-40 par des artisans italiens (Barbet et al., 1993 ; Loustaud, 2000, p. 186-188) ou par les peintures d'horti conclusi de III $^{\mathrm{e}}$ style tout pompéiens de Vaison-la-Romaine (fouille près de la cathédrale) ou de la Maison de la rue des Bouquets à Périgueux (Barbet et al., 2004, p. 154) (fig. 12).

Même si le corpus documentaire reste assez faible, on peut néanmoins constater que la qualité des peintures n'est pas toujours proportionnelle à ce que l'on s'attendrait à trouver; certaines maisons plus modestes que des maisons à péristyle peuvent ainsi en présenter de très grande qualité. Il n'en reste pas moins que, dans les maisons qui nous intéressent, le décor pictural le plus soigné et le plus luxueux se trouve presque toujours dans les diverses salles de réception.

Une idée du degré de standing des habitations transparaît à travers une hiérarchisation sommaire des formes et des décors picturaux utilisés (Barbet, 1980) : le haut de gamme est constitué par les compositions avec personnages (scènes mythologiques et historiques), puis, selon un ordre décroissant, on trouve les architectures (avec ou sans personnages), les figures volantes seules (pour les deux derniers styles pompéiens), les natures mortes, les candélabres et, enfin, les partitions géométriques. Outre le choix des motifs et leur qualité d'exécution, les matériaux employés interviennent également comme critère de différenciation, notamment lors du recours (souvent en grosses quantités) à des pigments coûteux tel le rouge cinabre (Pline, Histoire Naturelle, XXXV, 12 ; Barbet, 1983, p. 164 et 1990), ou, comme à Narbonne, à la feuille d'or (Sabrié et al., 1987, p. 192).

Longtemps restés les seuls témoignages archéologiques des maisons riches de Gaule, les pavements en mosaïque sont plus abondants et mieux conservés que tout autre type de décor. Même si leur caractère luxueux est nettement plus évident, ces tapis de sol semblent pourtant avoir été d'abord adoptés, en Narbonnaise, pour leur confort et leur aspect pratique, puis, de plus en plus, comme marqueur

69. La grande rareté des peintures de la première moitié du $\mathrm{II}^{\mathrm{e}} \mathrm{s}$. conservées en Narbonnaise (Sabrié et al., 1987, p. 186, par exemple) pourrait être due aussi bien à une lacune de la documentation qu'à une absence de réfection des décors antérieurs. 
social du fait de la dignitas qu'elles confèrent aux maisons qui en sont dotées (Lavagne, 1996). Malgré ce caractère, leur répartition chronologique, topographique ou géographique varie considérablement. $\mathrm{Si}$, très ponctuellement, quelques maisons en sont abondamment pourvues (Aix-enProvence, Alba-la-Romaine, Arles, Autun, Fréjus, Orange, Saint-Romain-en-Gal), la plupart n'en possèdent pas ou très peu ${ }^{70}$, sans que l'on soit assuré que la raison d'une telle rareté soit seulement financière ou due à une carence de fournisseurs. Le problème est encore plus sensible avec les décors en opus sectile, plus luxueux que la mosaïque (mais démontables) et associés aux maisons les plus riches. Présents dès le début du $\mathrm{I}^{\mathrm{er}} \mathrm{s}$. en Narbonnaise, leur emploi s'accroît considérablement dans la seconde moitié du $\mathrm{II}^{\mathrm{e}} \mathrm{s}$. et au début $\mathrm{du} \mathrm{III}^{\mathrm{e}} \mathrm{s}$. Même au sein des compositions géométriques assez simples du I ${ }^{\mathrm{er}}$ s., certains propriétaires réussissent cependant à se distinguer en recourant, par exemple, à des modèles inspirés de la décoration des palais impériaux, comme dans la Maison aixoise dite au Grand Péristyle dont la composition est visiblement inspirée de celle de la Domus Transitoria de Néron sur le Palatin (Collectif, 1996b, p. 36-37), ou au sein des exemples de la fin du Haut-Empire qui perpétuent la tradition géométrique et florale par le recours à des compositions figuratives (fig. 13). Le prestige de l'opus sectile est dû à celui du marbre (et autres roches décoratives assimilées) dont l'hégémonie esthétique semble avoir été telle qu'il paraît avoir été jugé comme étant la seule roche digne d'être visible dans une maison riche. En témoignent la peinture qui semble avoir systématiquement recouvert les pièces en briques stuquées et en calcaire ou autres roches moins nobles, ainsi que le succès durable des faux marbres peints, omniprésents dans les maisons. Pour la Gaule, on doit cependant noter que si le recours aux marbres exotiques (italiens, grecs, orientaux ou africains), à valeur hautement ostentatoire, paraît avoir été recherché, leur usage semble cependant avoir été quantitativement assez restreint, surtout au fur et à mesure que l'on progresse vers le nord. On a, en revanche, beaucoup eu recours au riche patrimoine géologique de la province qui produisait des marbres ou roches dures de grande qualité, notamment les Pyrénées (Mazeran, in Collectif, 1996a, p. 213-217 ; Lorenz et al. dir., 2000 ; Loustaud, 2000, p. 297-298 ; ChardronPicault et al. dir., 2004). Rien n'indique d'ailleurs que ces roches de substitution, plus économiques, n'aient pas

70. Une ville comme Vaison-la-Romaine, connue pour ses vastes maisons à péristyle, n'a ainsi livré qu'une douzaine de mosaïques et, sur la soixantaine de pièces de la Maison au Buste d'Argent, seule une en est dotée (Carru, in Collectif, 1996b, p. 339).

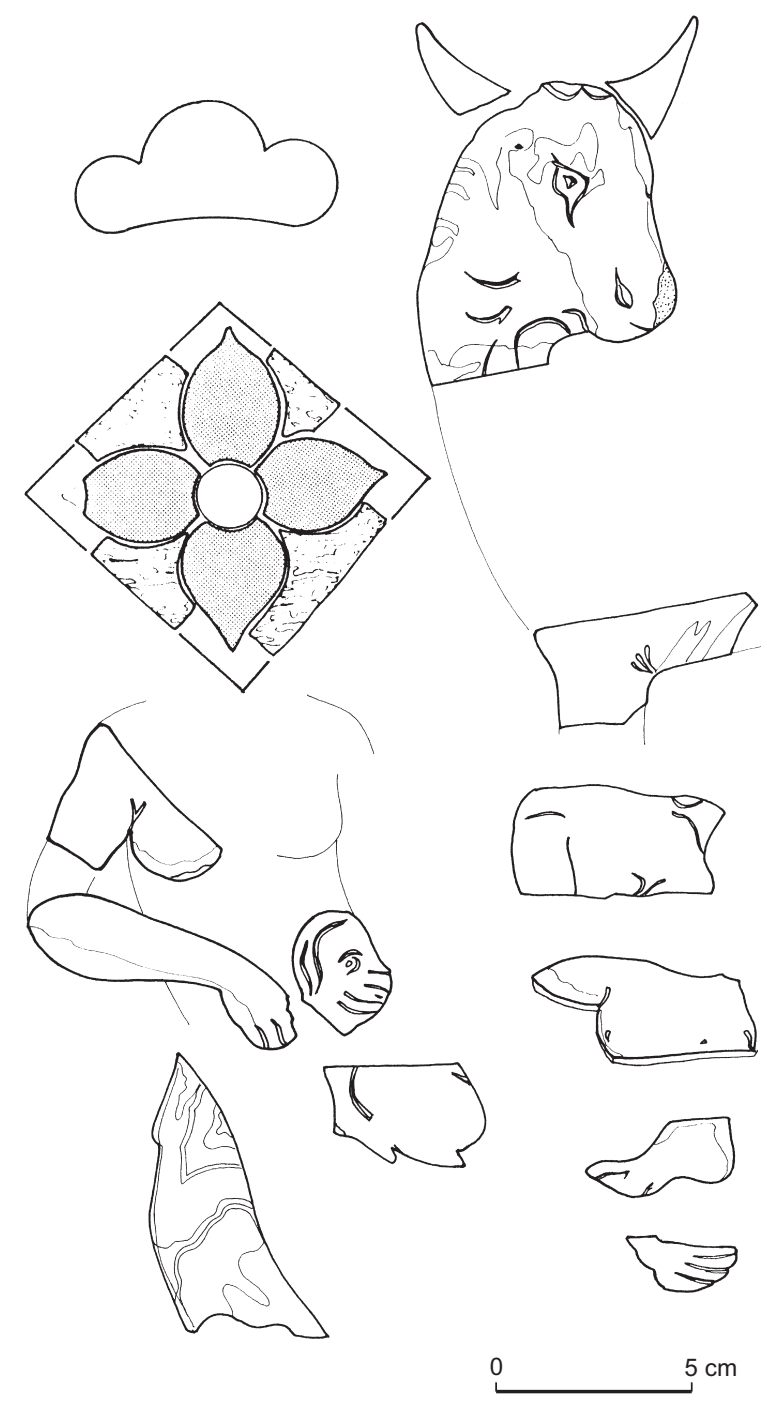

Fig. 13 - Éléments du décor en opus sectile d'époque sévérienne $d u$ viridarium de la Maison des Nones de Mars à Limoges (Haute-Vienne) (d'après Loustaud, 1992, fig. 1 et 2, p. 109-110).

suscité une certaine fierté auprès d'une clientèle gauloise dont le patriotisme est bien connu. Ce serait peut-être l'une des raisons de la diffusion souvent lointaine, vers le bassin Parisien et le Nord-Ouest - et même jusqu'en Bretagne insulaire -, d'un schiste noir produit par la cité d'Autun, le plus grand foyer de culture romaine de Gaule, par exemple (Vipard, Savary, 2004).

En ce qui concerne les colonnes, on entrevoit un usage quasi exclusif des colonnes appareillées, en briques stuquées ou en tambours de pierre (marbre, calcaire, granite). Cette technique, plus facile à mettre en ouvre et moins coûteuse, a sans doute joué un rôle capital dans la diffusion du péristyle en Gaule. 
Si le Sud connaît quelque usage de l'ordre corinthien, c'est assez systématiquement, et jusqu'en Bretagne, un ordre toscan provincial qui est utilisé, présentant une infinité de variantes de détails stéréotomiques (Broise, 1969). Quel que soit leur ordre, à chaque fois qu'on peut le constater, ces colonnes sont fortement inspirées par les formes de l'architecture publique locale.

Pour un grand nombre de ces colonnes, un décor peint (pour les roches autres que le marbre) n'est pas à exclure, mais l'envahissement éventuel de la surface par un décor en faible relief apparaît nettement seulement dans le cours $\mathrm{du} \mathrm{II}^{\mathrm{e}} \mathrm{s}$. (feuilles imbriquées, motifs d'inspiration dionysiaque). Ce décor, attesté dans des maisons d'Aquitaine ou de Lyonnaise (Bourges, Vieux et Rouen), n'est original que par sa technique et son emplacement, mais ne constitue en fait qu'une manifestation de ce décor végétal couvrant dont on trouve, à la même époque, des traces dans le IV e style pictural de Narbonnaise (Maison du Clos de la Lombarde, par exemple) ou, surtout, sur des mosaïques d'Aquitaine.

Considérant l'ensemble des techniques décoratives, on ne constate pas de style véritablement propre à une zone déterminée, mais plutôt l'existence d'aires se caractérisant par des traitements différents au sein d'un système globalement uniforme. Si certains motifs ou types de décors sont quelquefois caractéristiques d'une aire spécifique et propres à certains ateliers bien définis (mosaïstes aixois, d'Aquitaine, de la vallée du Rhône, par exemple), aucune zone n'est jamais entièrement isolée sur le plan artistique et l'on constate plutôt des aires de répartition dont les franges s'interpénètrent plus ou moins profondément suivant la technique ou le motif utilisé. L'itinérance des ateliers et le renom de certains d'entre eux ont eu un rôle apparemment uniformisateur dans l'expansion, peut-être relayés par des copistes locaux, de certains types d'œuvre. Un des cas les plus flagrants est peut-être celui des ateliers mosaïstes rhodaniens dont les œuvres sont très profondément dispersées en Lyonnaise, jusque dans l'actuelle Normandie et jusqu'en Britannia.

Chaque maison possède un décor unique, mais il est bien rare que chaque pièce de ce décor, prise individuellement, ne trouve pas son homologue dans une autre demeure contemporaine. Il serait trop long d'en administrer la preuve ici, mais le cas se vérifie facilement avec certaines peintures de bassins avec frises de poissons (Lisieux, Vieux, Périgueux, Limoges), certains types de motifs de mosaïque (notamment le décor de nid d'abeille déterminé par des bandes d'étoiles à six losanges) ou des colonnes à décor de panneaux dans la partie inférieure... Ce sont ces multiples points de comparaison, liés aux éléments architecturaux communs à toutes ces maisons, qui font que leur parenté est si évidente à travers la Gaule.

Malgré les problèmes de perception que pose son caractère extrêmement lacunaire, le décor luxueux est néanmoins toujours une valeur obligée des domus à péristyle. Sa fonction est bien évidemment esthétique, mais c'est avant tout la base d'un langage sans doute très élaboré jouant un rôle signalétique (hiérarchisation des espaces et des circulations dans la maison) et politique (officialisation de la maison, promotion individuelle du maître). Dans les parties les plus publiques, il est servi par les vastes dimensions des espaces, nécessaires à sa bonne vision, et trouve sa principale traduction dans des formes architecturales inspirées des formes publiques (et n'en différant quelquefois que par la taille). On y retrouve donc le transfert d'une partie du prestige de la publica magnificentia, réservée à l'édilité publique, dans la privata luxuria, légitimée par Vitruve dans l'habitat de la classe dominante qui exerce des fonctions politiques à l'intérieur de son habitation. Que ce soit par son plan ou par son décor, la typologie architecturale témoigne de l'importance du rôle politique assumé par la maison.

Si le rôle signifiant du décor - jamais univoque mais polysémique suivant la position dans l'édifice et le public est une donnée acquise, il faut avouer que son déchiffrement en est encore à ses balbutiements malgré de notables progrès et que l'on est en mesure de comprendre seulement une faible partie des valeurs qu'il exprime (Moormann dir., 1993 ; Perrin, 1997).

Le décor seul ne suffit généralement pas, hormis dans certains cas particuliers ${ }^{71}$, à identifier précisément la pièce qu'il orne; il ne peut être compris et prendre toute sa signification que dans son contexte. Il n'en reste pas moins qu'il est adapté à l'endroit qu'il orne. On a, par exemple, très justement fait remarquer que le $\mathrm{II}^{\mathrm{e}}$ style, avec ses décors théâtraux (contraires aux valeurs du mos majorum) et ses réminiscences royales, ne se trouvait pas dans les pièces publiques mais seulement dans les parties plus intimes (Perrin, 1997, p. 359) ; cela se vérifie dans un certain nombre de cas en Gaule, mais l'identification de trop de pièces reste indéterminée pour que l'on soit assuré d'une stricte application de ces préceptes ${ }^{72}$.

71. Comme les mosaïques en $\mathrm{U}$ ou en $\mathrm{T}$ qui désignent des triclinia, archéologiquement peu attestés au nord de la Loire.

72. On remarquera, par exemple, que les bassins polylobés, également censés représenter des frontes scenae, sont pourtant présents dans de nombreux jardins, peut-être du fait de la double nature de ces 
Du décor des maisons de Gaule se dégage finalement une impression de conformisme, typique des valeurs universelles dont il est porteur. Même dans les parties plus intimes, où l'imagination et l'expression culturelle propre du maître peuvent s'exprimer (Perrin, 1997, p. 358), tout reste néanmoins empreint d'une certaine solennité, d'un souci de dignitas. Ainsi ne semble-t-on encore avoir jamais trouvé dans une maison à péristyle une de ces scènes parodiques faisant intervenir des figures plus ou moins grotesques comme on en connaît pourtant dans des maisons moyennes ${ }^{73}$. On a souvent écrit que les commanditaires auraient rarement été conscients des modèles utilisés dans la décoration de leur maison (Clarke, 1991, p. 369, par exemple), et c'est sans doute vrai pour des Gaulois frâ̂chement romanisés, mais cela n’implique néanmoins pas nécessairement une réception entièrement passive des motifs. Parmi ceux qui leur étaient proposés, un choix était toujours possible qui, à travers les diverses possibilités d'interprétations des œuvres, permettait à qui le souhaitait de satisfaire ou d'exprimer des aspirations ou une culture personnelle éventuelles. Il ne faut pas oublier qu'avec le modèle architectural et politique de la maison à péristyle, c'est également son contenu culturel qui a dû être transplanté et développé en Gaule. Or dans le cadre de l'otium, la maison est notamment conçue comme un musée. Les composantes décoratives de celui-ci, destinées à exalter le mos majorum, sont également prétextes à des discussions ou à la réflexion (Lavagne, 1996, p. 46) dont ne sont pas exclues non plus des préoccupations éducatives et, notamment, morales. Tous ces aspects de la signification du décor s'interpénètrent, à l'image des diverses fonctions qui s'entremêlent au sein de la maison. Chaque œuvre est donc susceptible d'être interprétée de plusieurs manières en fonction de son contexte et de clefs souvent perdues. Les tableaux mythologiques ou autres scènes figurées se prêtent particulièrement à cet exercice (Lancha, 1997). Pour ne prendre qu'un exemple particulièrement représentatif : le thème du combat de Darée et Entelle sur des mosaïques de triclinium $\mathrm{du} \mathrm{II}^{\mathrm{e}} \mathrm{s}$. d'au moins trois maisons d'Aix-en-Provence, propre à un atelier de cette cité, concerne une scène tirée de l'Énéide (vers 424-484), rarement illustrée (fig. 14).

derniers: à la fois partie intégrante du péristyle en tant qu'espace public, mais également éléments indispensables à la satisfaction de l'otium.

73. Par exemple, dans la Maison 10 du site de Villa Roma à Nîmes (Sabrié et al., 1987, p. 192 et p. 194) ou à Saintes (Barbet et al., 1996, p. $\left.40, \mathrm{n}^{\mathrm{o}} 30\right)$.

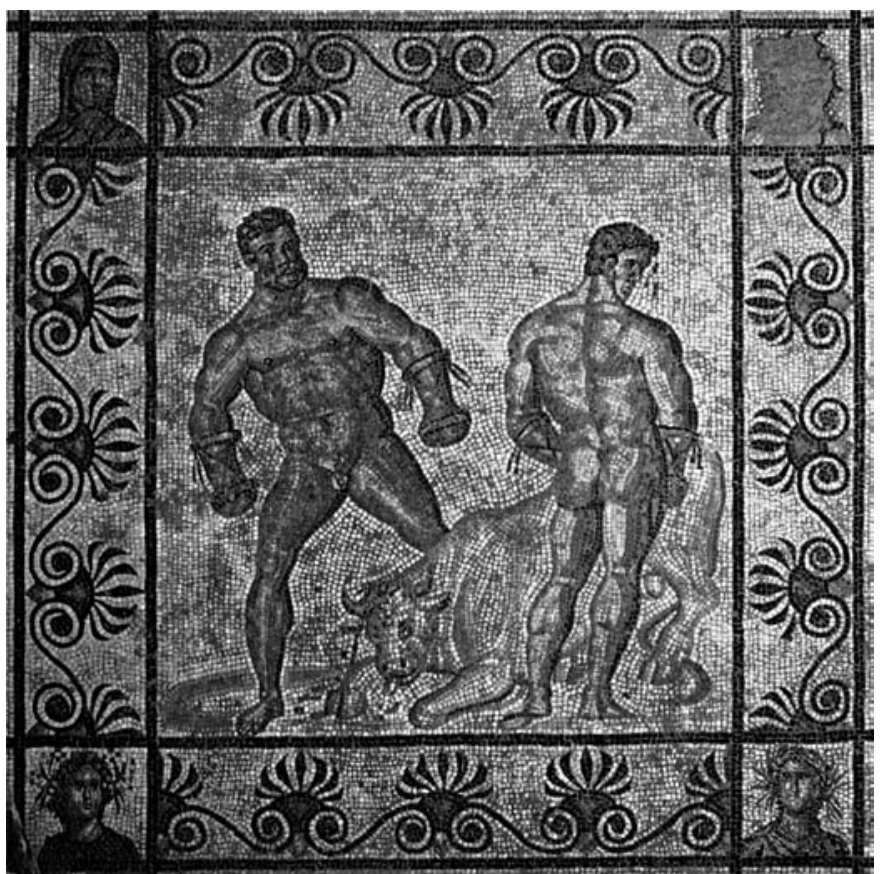

Fig. 14 - Le combat de Darée et Entelle sur une mosaïque du triclinium de la Maison de la rue des Magnans à Aix-en-Provence (Bouches-du-Rhône) (d'après Lavagne, 1994, fig. 1, p. 202).

Outre l'évocation très conformiste d'une scène épique, affichant peut-être ouvertement le goût des commanditaires pour l'œuvre la plus romaine de la littérature latine et leur appartenance à la romanité, l'une des raisons de ce choix paraît fort probablement morale : la résistance des hommes à la démesure ${ }^{74}$. Mais rien n'interdit non plus de supposer, plus prosaïquement, un goût particulier du propriétaire pour le pugilat, de même peut-être que pour les nombreux cas de représentations théâtrales, de scènes de courses de chars ou de combats de gladiateurs trouvés dans les villae, mais également dans quelques maisons urbaines. Les décors peints très conformistes du $\mathrm{I}^{\mathrm{er}} \mathrm{s}$. ne permettent cependant guère de déceler de tels choix, sauf peut-être à travers certains tableaux du $\mathrm{III}^{\mathrm{e}}$ style, mais les possibilités deviennent plus importantes avec le IV style et les libertés plus grandes qu'il permet, notamment dans ses développements avec de grandes scènes à personnages où les propriétaires semblent alors avoir eu une plus grande possibilité d'exprimer leur sensibilité ou leur personnalité propre, tout

74. Voir Lavagne, 1994 et 1996, p. 46 (avec d'autres exemples). Un autre cas d'œuvre à interprétation morale (la démesure punie, la nécessité du respect de l'autorité) est sans doute la figuration de Marsyas sur le point d'être écorché sur un des bas-reliefs du vestibule de la Maison au Grand Péristyle de Vieux (Vipard, 2001, p. 24, fig. 6). 
en respectant les conventions (Vipard, 2004). Attestées dès la seconde moitié ou la fin du $\mathrm{I}^{\mathrm{er}} \mathrm{s}$. dans la Maison de la rue des Bouquets à Périgueux ${ }^{75}$, elles ne deviennent toutefois vraiment attestées que vers la fin de la période des Antonins et sous les Sévères en Gaule, sans doute sous l'effet d'une évolution des mentalités (leur présence pourrait également s'expliquer dans le cadre des activités d'une éventuelle schola présente dans la maison).

Ces grandes scènes, très ostentatoires, permettent, pour la première fois sans doute, une réelle autocélébration du dominus qui a désormais la possibilité de représenter un thème qui lui est cher ou de commémorer un événement marquant de sa vie publique ou privée - ses res gestae -, par exemple dans la scène isiaque de la Maison de la place des Épars à Chartres (Allag, 1993) (fig. 15).

Ces scènes n'exaltent pas seulement le propriétaire, mais perpétuent en fait toujours l'affirmation de sa romanité et l'expression sincère de sa fidélité à l'Empire, notamment à travers le culte impérial. En témoignent la scène au Génie et à la Victoire du Clos de la Lombarde à Narbonne (Sabrié et al., 1987, p. 204-209, fig. 169-173 et Sabrié, Sabrié, 1999) ${ }^{76}$ (fig. 16), mais également les scènes, d'inspiration orientale, empreintes de la religiosité en vogue dans les classes dirigeantes à cette époque (Chartres et Vieux, par exemple). Cette pratique nouvelle, loin d'être révolutionnaire, correspond cependant à une évolution des sensibilités aristocratiques, peut-être sous l'effet de l'arrivée dans les rangs des élites d'homines novi. Si elle peut être considérée comme un trait provincial (avec la notion de condescendance qui s'y attache), Rome même ne semble pas y avoir échappé ${ }^{77}$. Elle s'inscrit dans le courant de hausse considérable du luxe qui caractérise les maisons gauloises au cours de la seconde moitié du $\mathrm{II}^{\mathrm{e}} \mathrm{s}$. et au début du $\mathrm{III}^{\mathrm{e}} \mathrm{s}$., et qui se manifeste par des campagnes de décoration

75. Ces gladiateurs grandeur nature (et les scènes de venatio) du mur sud du péristyle sont donc contemporains du Satiricon de Pétrone, d'époque néronienne, où les res gestae des diverses étapes de la vie de l'affranchi Trimalcion ornent les murs de son atrium (voir notamment Dumont, 1990). Sous prétexte de la connotation "nouveau riche » de ce type de décor, on a utilisé cet hapax pictural pour appuyer la thèse d'un monument à destination publique (voir note 13, p. 229), mais que sait-on de la sensibilité des provinciaux gaulois à cette époque?

76. Les Tutèles, comme les Génies, sont deux manifestations de loyalisme impérial. La présence de certains traits de décor empruntés à des thèmes officiels rattachables à l'idéologie impériale du moment (gigantomachie, prisonniers orientaux ou barbares) sur des bas-reliefs de Vieux iraient dans le même sens (Vipard, 2001, p. 32), de même qu'une scène peinte de dextrarum junctio de Paris (Eristov, 1994).

77. On en décèle en effet des traces au II $^{\mathrm{e}} \mathrm{s}$. à Rome même dans des maisons de nouveaux sénateurs (exemple dans Guilhembet, 2001, p. 232).

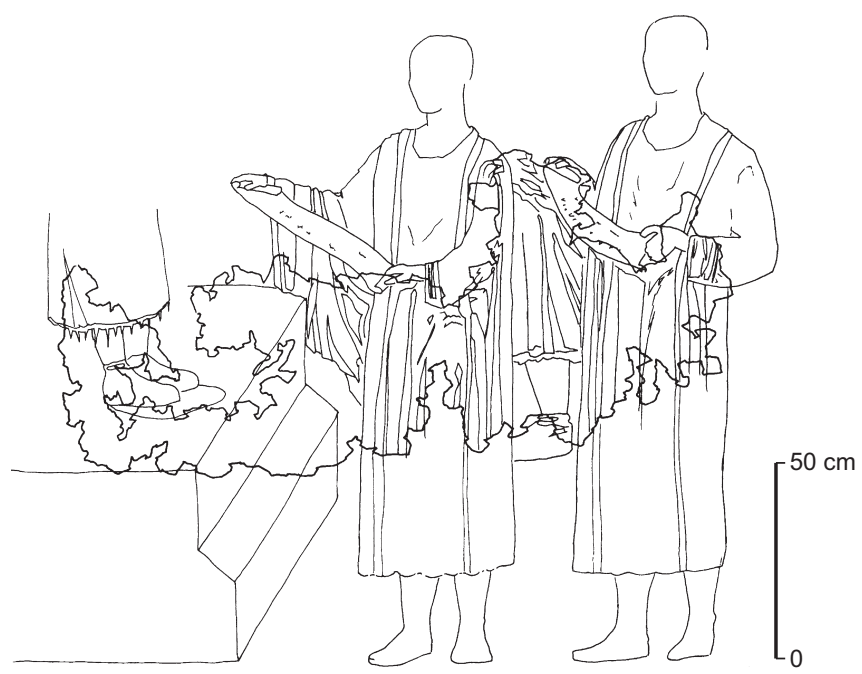

Fig. 15 - Fragment de cérémonie isiaque de la Maison d'époque sévérienne de la place des Épars à Chartres (Eure-et-Loir) (d'après Allag, 1993, fig. 3).

archéologiquement constatées partout. Le rôle jusqu'alors primordial de la peinture, sans perdre de son importance, diminue néanmoins devant la diversification des techniques. Comme auparavant, les motifs et thèmes décoratifs ne sont cependant pas originaux, mais tout à fait conformes à ceux alors en vogue dans tout l'Empire et dans tous les domaines de l'architecture (notamment avec la hausse des éléments d'inspiration dionysiaque et orientalisants). Pour les maisons anciennement constituées, dont le décor était généralement assez sobre, cette hausse décorative ne s'accompagne d'ailleurs pas toujours d'une campagne importante de transformations architecturales ni ne consiste en un renouvellement complet du décor architectonique, mais procède souvent par ajouts et juxtapositions à l'existant. On peut ainsi constater un accueil de modes nouvelles, conciliable avec la tendance au conservatisme qui s'est très rapidement fait jour au $\mathrm{I}^{\mathrm{er}} \mathrm{s}$. (en peinture essentiellement) qui a perduré. Plusieurs cas montrent en effet que l'on a souvent volontairement conservé ce qui pouvait l'être ${ }^{78}$. On pourrait d'ailleurs également se demander si certaines réapparitions de formes architecturales, disparues depuis longtemps, telles des entrées de type vestibulum-fauces, des atria ou des tablina, ne participeraient pas d'une même réaction archaïsante, les formes anciennes conférant une dignitas certaine à la maison et au dominus.

78. La longue utilisation des décors ne doit donc pas être interprétée (du moins systématiquement) comme une impossibilité financière à renouveler la décoration. 


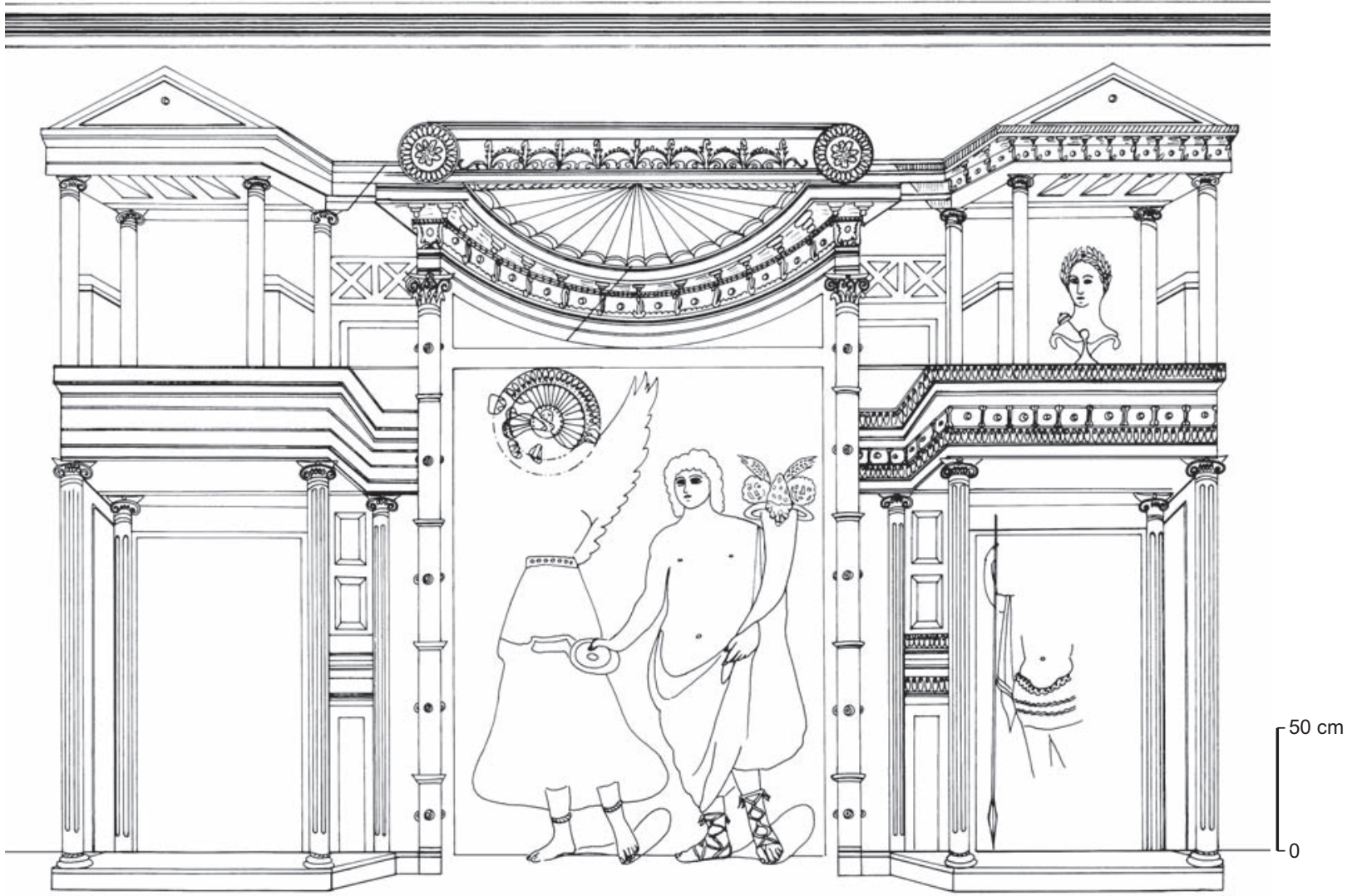

Fig. 16 - Décor du triclinium $K$ de la Maison du Clos de la Lombarde à Narbonne (Aude), seconde moitié du II ${ }^{e}$ s. (d'après Sabrié, Sabrié, 1999).

Cette hausse considérable de la parure intérieure s'explique en grande partie par la bonne santé économique des élites à l'époque des Antonins et des Sévères, sans doute aussi par une surenchère ostentatoire sans cesse croissante dans laquelle, renversant complètement les valeurs établies à la fin de la République, le luxe suppléait désormais à la dimension de la domus (difficile à accroître). Cette hausse du luxe a également été rendue possible par l'existence d'ateliers capables de satisfaire la demande. En ce qui concerne l'irruption du matériel lapidaire sculpté dans la maison, par exemple, on a quelquefois constaté qu'elle coïncidait avec la fin des grands travaux édilitaires publics dans le courant $\mathrm{du} \mathrm{II}^{\mathrm{e}} \mathrm{s}$. et que les ateliers auraient alors pu recentrer une partie de leur activité vers l'architecture privée, domestique et funéraire (Tardy, 1992, p. 332), d'autres fois, au contraire, elle est concomitante d'importants travaux tardifs de constructions publiques ${ }^{79}$.

79. C'est notament le cas de la Maison au Grand Péristyle à Vieux, contemporaine de la construction des « Thermes Sud».

\section{Dimension et instabilité des domus}

Comme il l'est encore aujourd'hui pour l'archéologue, le gigantisme de la plupart des maisons à péristyle par rapport au reste de l'habitat, voire par rapport aux monuments publics, était sans doute la caractéristique la plus immédiatement perceptible par les contemporains (tabl. III).

Répondant, certes, à la nécessité matérielle de loger convenablement et dignement la famille du maître et son personnel, de recevoir amis et relations diverses, l'ampleur des maisons à péristyle semble cependant surtout liée au besoin d'occuper ostensiblement un espace et, surtout, de favoriser le développement du péristyle.

Une première preuve en est la prédilection pour l'extension horizontale. On n'a en effet aucune preuve, même dans le centre-ville, là où la densité de construction était la plus forte, d'une recherche d'extension verticale. Dans le meilleur des cas, l'archéologie révèle l'existence d'un premier étage, mais jamais encore de deux. Cet étage semble d'ailleurs avoir été souvent incomplet, localisé sur un 
Tabl. III - Surfaces de maisons à péristyle de Gaule, de Germanie et de Bretagne: $S^{2}$, superficie estimée ou minimale en mètres carrés.

\begin{tabular}{|c|c|c|}
\hline Localisation & Dénomination & $\mathbf{S}^{2}$ \\
\hline Limoges (Haute-Vienne) & Maison sous I'Insula VI-5 & 5500 \\
\hline Saint-Romain-en-Gal (Rhône) & Maison sous le Lycée & 4600 \\
\hline Limoges (Haute-Vienne) & Maison des Nones de Mars & 3735 \\
\hline Vaison-la-Romaine (Vaucluse) & Maison au Buste d'Argent & 3500 \\
\hline Vaison-la-Romaine (Vaucluse) & Le Prétoire & 3500 \\
\hline Bibracte (Saône-et-Loire) & Maison PC 1 & 3500 \\
\hline Cologne (Allemagne) & Maison de la Mosaïque de Bacchus & 3400 \\
\hline Fréjus (Var) & Maison de la Plate-Forme & 3290 \\
\hline Saint-Romain-en-Gal (Rhône) & Maison au Lion & 3100 \\
\hline Augst (Suisse) & Maison de I'Insula 30 & 3000 \\
\hline Saint-Romain-en-Gal (Rhône) & Maison des Dieux Océan, états 3 et 4 & 2930 \\
\hline Vaison-la-Romaine (Vaucluse) & Maison au Dauphin & 2700 \\
\hline Avenches (Suisse) & Maison de I'Insula XIII Est & 2700 \\
\hline Aix-en-Provence (B.-du-Rhône) & Maison de l'Aire du Chapitre & 2600 \\
\hline Périgueux (Dordogne) & Maison à I'Impluvium Polylobé & 2500 \\
\hline Avenches (Suisse) & Maison de I'Insula XIII Ouest & 2400 \\
\hline Périgueux (Dordogne) & Maison de la rue des Bouquets & 2350 \\
\hline Limoges (Haute-Vienne) & Maison à l'Opus Sectile & 2300 \\
\hline Limoges (Haute-Vienne) & Maison à l'Emblema au Lion & 2300 \\
\hline Vaison-la-Romaine (Vaucluse) & Maison des Messii & 2100 \\
\hline Saint-Romain-en-Gal (Rhône) & Maison du Vivier & 2000 \\
\hline Caerwent (Grande-Bretagne) & Maison de l'Insula IIIS & 1980 \\
\hline Silchester (Grande-Bretagne) & Maison à Corridor de I'Insula XIV, 1 & 1700 \\
\hline Caerwent (Grande-Bretagne) & Maison à Corridor de I'Insula VII & 1620 \\
\hline Wroxeter (Grande-Bretagne) & Maison à Corridor VI & 1615 \\
\hline Nyon (Suisse) & Maison de Bel-Air & 1600 \\
\hline Limoges (Haute-Vienne) & Maison au Grand Vestibule & 1600 \\
\hline Avenches (Suisse) & Maison de I'Insula XVI Est & 1570 \\
\hline Cologne (Allemagne) & Maison à Atrium de la Cathédrale & 1440 \\
\hline Vieux (Calvados) & Maison au Grand Péristyle & 1420 \\
\hline Alba-la-Romaine (Ardèche) & Maison du Champ Delauzun & 1420 \\
\hline Martigny (Suisse) & Maison au nord du Forum & 1400 \\
\hline Metz (Moselle) & Maison de l'îlot B du site de l'Arsenal & 1350 \\
\hline Saint-Romain-en-Gal (Rhône) & Maison des Dieux Océan, état 2 & 1326 \\
\hline Fréjus (Var) & Maison du Clos de la Tour & 1090 \\
\hline Bibracte (Nièvre) & Maison PC 2 & 1040 \\
\hline Narbonne (Aude) & Maison du Clos de la Lombarde & 975 \\
\hline Rezé (Loire-Atlantique) & Maison sud du Terrain Peigné & 880 \\
\hline Lyon (Rhône) & Maison aux Masques & 800 \\
\hline Le Mans (Sarthe) & Maison des Halles & 690 \\
\hline Lyon (Rhône) & Maison aux Xenia & 660 \\
\hline Saint-Romain-en-Gal (Rhône) & Maison aux Cinq Mosaïques & 500 \\
\hline Amiens (Somme) & Maison 1 du Palais des Sports & 465 \\
\hline Saint-Raphaël (Var) & Maison du Suveret & 460 \\
\hline
\end{tabular}

ou deux côtés de la maison seulement, sans doute pour des raisons de luminosité et donc de confort, l'élévation étant souvent difficilement compatible avec la pénétration de la lumière au rez-de-chaussée.

Une seconde preuve est constituée par le fait que, dans le cas d'une maison grande dès sa construction, la surface occupée par le péristyle par rapport aux salles est proportionnellement très grande et que, dans le cas d'un agrandissement ultérieur, on pourrait dire qu'elle croît exponentiellement. Il faut donc supposer que les maisons recherchaient une vaste amplitude pour avoir de grands péristyles.

Du fait du décalage chronologique entre les débuts de l'urbanisation et l'implantation de nombreuses maisons à péristyle, cette recherche d'espace s'est rapidement heurtée à la raréfaction de ce dernier, souvent dès le $\mathrm{I}^{\mathrm{er}} \mathrm{s}$. Si certains notables ont peut-être été contraints d'aller s'installer dans des quartiers périphériques où la place était disponible, la majorité des cas montre au contraire une installation au plus près du centre civique, après acquisition et destruction de constructions permettant d'obtenir des lots suffisamment grands dont l'agrandissement ne cessait qu'à cause de l'obstacle d'un bâtiment public ou d'une autre grande domus, quelquefois même avec l'atteinte des limites de l'insula elle-même ${ }^{80}$. Sous le Haut-Empire, l'outrepassement de ces dernières par empiétement sur le domaine public, en l'occurrence les rues publiques, semble avoir été rarissime en Gaule. Contrairement à ce qui se passe dans d'autres provinces, aucun cas d'annexion de trottoir n'est clairement attesté. Le degré d'illégalité d'une telle pratique est d'ailleurs difficile à mesurer puisque le statut juridique de cet espace, qui semble avoir appartenu aux riverains mais avoir été (ou dû être) laissé à la disposition du public, n'est pas bien connu.

De nombreuses maisons n'occupent cependant qu'une partie de l'insula, soit que les propriétaires n'aient pu mener leur politique d'annexion à terme, soit qu'ils aient estimé avoir suffisamment d'espace. Une fois l'équilibre atteint entre les besoins de fonctionnement et de représentation, la dimension de la domus semble en effet avoir moins compté que le luxe, constatation également valable dans le cas de petites maisons ne pouvant pas être agrandies.

En règle générale, il ressort également des fouilles récentes que les opérations d'agrandissement ne s'effec-

80. Par exemple, dans le cas à Limoges, avec les Maisons de l'Opus Sectile et de l'Opus Quadratum qui ne vont plus former qu'une seule construction dans la première moitié du $\mathrm{II}^{\mathrm{e}} \mathrm{s}$., ou avec les deux domus de $2700 \mathrm{~m}^{2}$ et $2400 \mathrm{~m}^{2}$ qui occupent à elles deux toute l'Insula XIII d'Avenches (Germanie Supérieure). 
tuaient pas toujours en une seule phase, mais consistaient souvent, à partir d'une construction existante, dans la fusion plus ou moins progressive et rapide de lots voisins. Ces mêmes fouilles font également apparaître que ces opérations s'accompagnent de travaux de restructuration ou de réfection, mais qu'à la même époque, l'activité décorative est tout aussi intense dans les maisons qui ne peuvent plus s'agrandir 81 .

Malgré les fortes tendances conservatrices de ce type d'habitat, l'immuabilité n'était donc pas la règle et, au contraire, une certaine instabilité peut être tenue pour une des caractéristiques importantes des maisons à péristyle. Cet état d'impermanence du cadre domestique, mais surtout sa construction par à-coups, pour ne pas dire par tâtonnements dans certains cas, en fonction des possibilités financières et des visées des propriétaires, explique l'absence d'uniformité qui caractérise le plan de ces maisons. La périodicité, très variable d'une maison à l'autre, entre les diverses phases de transformation s'explique sans doute par la réalisation d'un équilibre entre les services attendus et les besoins et les buts des propriétaires. Qu'elles s'opèrent dans le cadre de limites déjà existantes ou à l'occasion d'un agrandissement par fusion, toutes les transformations architecturales et décoratives de l'espace intérieur, dont la fréquence est souvent inférieure à une génération, peuvent sans doute être interprétées comme la traduction architecturale de la montée en puissance sociale d'une famille de notables ou d'un propriétaire.

Du fait de leur importance volumétrique, de la construction ou des campagnes de transformations successives, ces travaux devaient d'ailleurs contribuer à attirer l'attention sur leur commanditaire et les maisons à péristyle ont souvent dû conférer aux villes gauloises, du I ${ }^{\mathrm{er}} \mathrm{s}$. jusqu'au début du $\mathrm{III}^{\mathrm{e}} \mathrm{s}$. quelquefois, l'allure de chantiers permanents.

Posséder une très grande demeure était donc apparemment nécessaire à l'affirmation de la puissance des notables, mais, comme nous l'avons souligné, la taille ne semble plus avoir été un problème quand un point d'équilibre entre surface et besoins de logement, de service et de représentation, était atteint. Une fois encore le travail sur les plans empêche peut-être d'entrevoir une partie de la réalité antique en focalisant notre attention sur la

81. Même la Maison des Nones de Mars à Limoges, dont la taille n'a jamais évolué depuis sa création vers 35-45 apr. J.-C., connaît des remaniements internes de son plan et des changements de décoration dans le dernier tiers du I ${ }^{\mathrm{er}} \mathrm{s}$. ainsi qu'une série d'autres changements encore plus profonds entre le milieu du $\mathrm{II}^{\mathrm{e}} \mathrm{s}$. et le milieu du III ${ }^{\mathrm{e}} \mathrm{s}$. (Loustaud, 1992, p. 68-69 et p. 71). dimension objective des demeures au détriment de leur taille subjective : en effet, au-delà de $1000 \mathrm{~m}^{2}$ ou plus, il ne devait souvent pas être possible de distinguer les unes des autres les maisons qui différaient seulement de quelques centaines de mètres carrés; une maison plus petite (en plan) que sa voisine n'était sans doute pas nécessairement ressentie comme moins imposante. Ce sont les possibilités plus ou moins importantes de valoriser, de mettre en scène l'extérieur de la construction qui devaient permettre de traduire la haute situation sociale de son occupant en accroissant l'impression de grandeur. L'utilisation de la topographie, de la perspective, d'aménagements architecturaux (inspirés des monuments publics) devaient permettre au propriétaire, selon ses moyens financiers, de faire paraître sa demeure plus grande qu'elle n'était réellement. De même, en ce qui concerne l'intérieur, la dimension des espaces visibles par les visiteurs ayant accès à telle ou telle partie de la maison devait revêtir une certaine importance pour le prestige du maître, mais la décoration et l'agencement des lieux devaient être au moins aussi importants, contribuer, tout comme l'aspect extérieur de la domus, à son renom et à lui conférer une grandeur psychologique, par l'intermédiaire des commentaires plus ou moins subjectifs que les privilégiés ne devaient pas manquer de rapporter à ceux qui n'étaient pas admis à l'intérieur.

De ce point de vue, la mediocritas de certaines domus par rapport aux dimensions de leurs homologues doit vraisemblablement être tempérée par leur position dans une cité plus modeste, et surtout par l'abondance et le luxe de leur décor.

\section{Les caractères publics de l'architecture domestique}

Les caractères publics, décelables dans certaines parties du plan ou du décor de l'architecture domestique et auxquels on a déjà eu l'occasion de faire allusion à maintes reprises, constituent en quelque sorte le point commun à toutes les caractéristiques fondamentales précédemment traitées.

Ces caractères ne devaient pas peu contribuer à assimiler les maisons à des monuments publics et leur accumulation et leur agencement sont tels que l'on peut difficilement supposer que les commanditaires n'aient pas consciemment utilisé ce mode d'expression privilégié pour traduire leur grandeur ou celle de leur famille.

Les rapports étroits entre les demeures des grands et l'architecture publique tels qu'ils ressortent de l'œuvre de Vitruve et de cas archéologiques ont été mis en évidence 
par de nombreux travaux pour la fin de l'époque républicaine ou le début du Haut-Empire. Hormis pour l'Afrique (Thébert, 1985), cette relation est nettement moins bien étudiée durant la suite du Haut-Empire dans les provinces, et à ce titre l'exemple gaulois apporte de nombreuses évidences qui montrent la poursuite de cette pratique.

Il n'est pas question de reprendre l'ensemble des points qui constituent des rapprochements entre les architectures publique et privée, ni de toujours entrer dans le détail, mais simplement de pointer très brièvement ceux que l'on est susceptible de trouver en Gaule et qui montrent que ses notables ont très vite compris comment en tirer avantage.

L'élément le plus immédiatement perceptible est sans doute celui (qui va être évoqué plus loin) de la grande taille des maisons, souvent supérieure à la majeure partie des bâtiments publics de la cité. Moins sensible peut-être pourrait avoir été la régularité (au moins apparente) de leur plan qui les rapprochait également des monuments publics souvent bâtis d'un seul jet, sur un terrain libre ou libéré de toute contrainte. Il est un fait que les maisons les plus régulières (s'inscrivant dans un rectangle) sont très rares et sont généralement les plus riches (souvent aussi les plus anciennement implantées).

La forte monumentalisation de quelques entrées restituables va dans le même sens: notamment les frontons, comme par exemple ceux associés aux quatre colonnes des entrées des Maisons limousines des Nones de Mars (deuxième quart du I ${ }^{\mathrm{er}} \mathrm{s}$.) et à l'Opus Quadratum (première moitié du II ${ }^{\mathrm{e}}$ s.) (Loustaud, 2000, fig. 35 et fig. 60), forme immédiatement comprise comme étant le symbole du palais ou du temple par les contemporains ${ }^{82}$; ou encore les arches géminées, analogues aux représentations des portes de villes, dans la Maison au Grand Péristyle à Vieux (début du $\mathrm{III}^{\mathrm{e}}$ s.) (fig. 17), avec en outre, à l'arrière-plan du vestibule, ses pilastres ornés de bas-reliefs de scènes mythologiques (Vipard, 2001, p. 23-24, fig. 4 à 6). Monumentalisation renforcée dans chaque cas par l'intégration à la colonnade publique surélevée et dallée.

L'un des éléments capitaux de l'assimilation est précisément constitué par l'omniprésence des colonnes qui interviennent dès la mise en contact des espaces publics et privés par le biais des portiques de façade. Les colonnes ne sont en effet pas une simple méthode de construction, mais un élément architectural en rapport avec cette conception de l'officialisation de la demeure. Déjà signe distinctif de l'archi-

82. Voir Vitruve, De Architectura, V, 6, 9 (autres références - ${ }^{\mathrm{er}} \mathrm{s}$. av. J.-C.-II ${ }^{\mathrm{e}}$ s. apr. J.-C. - dans Guilhembet, 2001, p. 220, n. 20). tecture publique ou sacrée chez les Grecs, les Romains ont accentué ce caractère qui met les édifices privés et publics sur un même plan et dont le péristyle constitue bien évidemment le mode d'expression privilégié (Wallace-Hadrill, 1988, p. 66-67). L'allusion ne se borne pas au seul emploi d'une structure porteuse, mais également à ses formes tant stéréotomiques que décoratives. On a depuis longtemps constaté l'identité des premières pour les colonnes de Narbonnaise, lors de l'étude de la Maison au Dauphin à Vaison-la-Romaine (Goudineau, 1979, p. 211-212), et le fait se vérifie avec les colonnes toscanes que l'on rencontre dans les Trois Gaules et au-delà. On a un bel exemple de cette domestication de colonnes publiques avec celle de la Maison du «Bas de Vieux » qui trouvent en effet leurs répliques exactes dans celles des grands thermes voisins (même moulures, mêmes motifs décoratifs de surface). Des décors considérés jusqu'alors comme publics se retrouvent en fait dans des contextes domestiques de la seconde moitié du $\mathrm{II}^{\mathrm{e}} \mathrm{s}$. ou du début du III ${ }^{\mathrm{e}}$ s. dans l'ouest et le sud-ouest de la Gaule. C'est, par exemple, le cas des décors d'imbrications ou dionysiaques, jusqu'alors systématiquement rattachés à des temples ou à des monuments publics ou, pour les premières, à des colonnes de Jupiter à l'anguipède (Walter, 1970 ; Eristov, Vaugiraud, 1994 ou Bouet, 2001, p. 256, par exemple).

Dans quelque endroit domestique que ce soit, la présence de colonnes a pour effet de désigner cet espace comme prestigieux (Wallace-Hadrill, 1988, p. 68-70 et p. 76), ce pourrait donc être une des raisons de leur présence dans le décor peint.

Le décor et sa richesse sont un autre des caractères publics des domus riches. Le prestige des $\mathrm{II}^{\mathrm{e}}$ et $\mathrm{III}^{\mathrm{e}}$ styles ou celui des scènes figurées sont liés à l'association publique qui leur est attachée (Wallace-Hadrill, 1988, p. 75) et l'on se demande dans quelle mesure un certain nombre de ces grandes scènes commémoratives qui apparaissent dans le IV ${ }^{\mathrm{e}}$ style de la seconde moitié du $\mathrm{II}^{\mathrm{e}} \mathrm{s}$. et du début $\mathrm{du} \mathrm{III}^{\mathrm{e}} \mathrm{s}$. ne perpétuaient pas le parallèle entre maisons et temples de l'époque républicaine (Wiseman, 1987, p. 395, par exemple). Il convient également de rappeler, bien que les cas soient rares, le rôle des statues à caractère public marqué comme celui des Tutelae.

Il faut également citer d'autres éléments, comme la présence de fontaines, bassins ou jeux d'eau divers, indissociables du péristyle et liés à la présence d'eau courante dont l'octroi semble avoir toujours revêtu un aspect public.

On pourrait sans doute encore trouver de nombreux éléments montrant l'assimilation d'une maison à un monument public. Leur accumulation est trop abondante et 


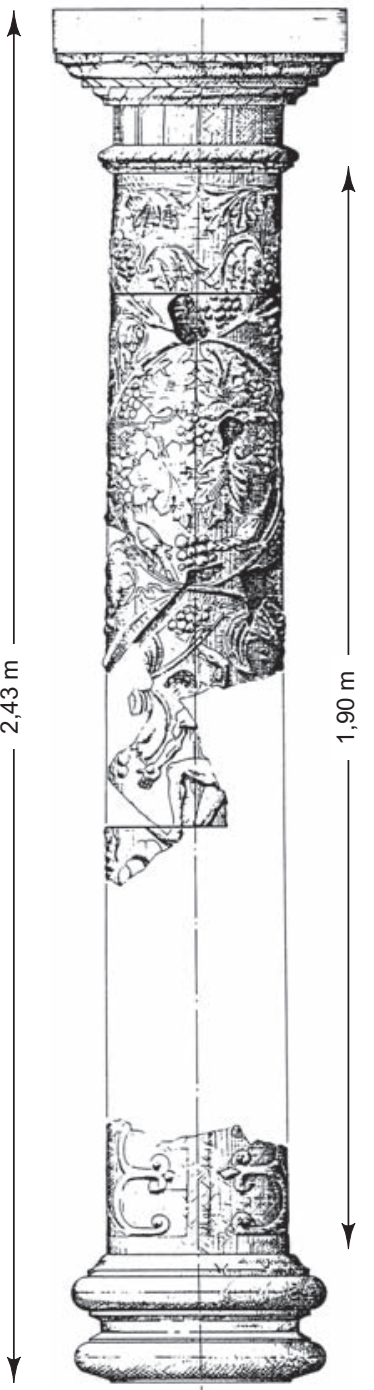

1

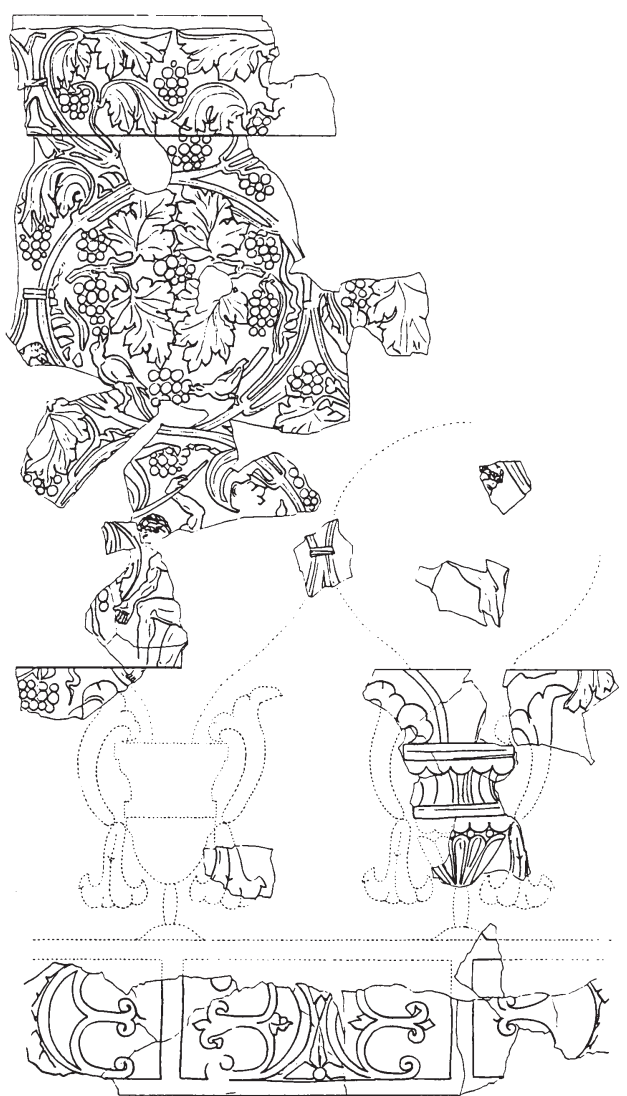

2

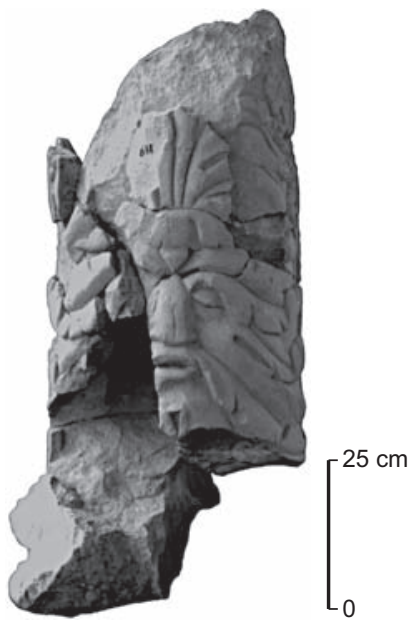

3

Fig. 17 - Exemple de matériel architectonique traditionnellement attribué à l'architecture publique : les colonnes à décor dionysiaque du côté nord du péristyle de la Maison au Grand Péristyle à Vieux (Calvados), vers 200 apr. J.-C. 1, 2, rinceaux de vigne peuplés et bacchant ivre ;

3, masque de génie végétal (d'après Vipard, 2001, fig. 8 et 9).

organisée pour avoir été inconsciente et il est donc difficile d'admettre que les propriétaires de province n'aient pas voulu, comme à Rome, utiliser leur maison à péristyle dans un dessein politique.

\section{QUELQUES COMPOSANTES CARACTÉRISTIQUES SECONDAIRES}

Leur présence n'étant pas aussi systématique ou constatable que celles précédemment étudiées, il est malaisé de mesurer le rôle ou la nécessité exacts d'un certain nombre de composantes architecturales ou plus immatérielles. Comme il est difficile d'en dégager des conclusions très précises, on se contentera de fournir quelques aspects très généraux.

Ainsi, une des grandes absentes de la maison telle que la restitue l'archéologie est la domesticité. Alors que l'on connaît par les sources écrites le rôle capital joué par le personnel, abondant et spécialisé, dans le fonctionnement et la renommée d'une maison, celui-ci nous échappe complètement et il faut reconnaître que l'on n'est pas en mesure d'identifier clairement, en dehors bien évidemment des espaces dits de service, dont le rôle est d'ailleurs souvent très flou, des espaces spécifiquement serviles et, notamment, les pièces destinées à l'hébergement de cette domesticité. Même 
si l'hypothèse paraît raisonnable, on ne possède absolument aucune preuve que l'étage (ou les combles), assez souvent attesté, ait pu servir de logement à une population servile. Les seuls indices positifs que l'on possède à ce sujet ne révèlent actuellement qu'une occupation noble ; les seuls endroits où l'on ressent très vaguement la présence des esclaves sont les salles ou espaces de service qui livrent rarement des éléments très parlants et varient sensiblement d'une maison à l'autre. À quelques exceptions près, les cuisines ne semblent pas avoir possédé un équipement très lourd ${ }^{83}$.

Les espaces résidentiels des maîtres, hormis ceux déjà étudiés, sont presque aussi mal connus. Quant aux éventuelles bibliothèques et pinacothèques, elles ne sont pas formellement identifiables. Tout au plus arrive-t-on à identifier des chambres à coucher : par des graffitis (Bost, 1993), des traces de lits sur le sol ou les murs (Barbet et al., 1996, p. 34), mais le plus souvent par intuition plus que par preuve matérielle.

Nettement plus intéressants sont les équipements de confort qui semblent avoir été d'usage courant ${ }^{84}$. Quoique difficile à observer, la présence de latrines particulières, quelquefois même chauffées et souvent liées à l'évacuation des eaux (Leveau, 1996, p. 160), est assez fréquemment constatée, mais souvent leur absence pourrait avoir été compensée par l'usage des seaux de chambre et d'un personnel spécialisé. Sans être rares, les thermes (plus ou moins complets) ne semblent pas très fréquents (Bouet, 1996). Dans les maisons précoces, on peut supposer qu'ils avaient pour but de pallier l'insuffisance des équipements publics ${ }^{85}$; plus tardivement, sous le Haut-Empire, cette présence s'explique éventuellement par un désir de liberté (souci d'éviter d'avoir à se déplacer ou de devoir respecter des horaires et d'échapper à la cohue, notamment lorsque l'on reçoit des invités, voir Pétrone, Satiricon, 72, 3 et $73,2)$, mais on constate cependant leur suppression dans certaines maisons pourtant à leur apogée comme celle à l'Opus Quadratum à Limoges (seconde moitié du $\mathrm{I}^{\mathrm{er}} \mathrm{s}$.), ou au Grand Péristyle à Vieux (fin du $\mathrm{II}^{\mathrm{e}} \mathrm{s}$.).

83. Plans de quelques cuisines gauloises dans Bouet, 2001, p. 251, fig. 14 (sur les raisons probables de la simplification des installations, id., p. 252).

84. Certains faisant intervenir une technologie avancée, comme les pompes hydrauliques, ont un rôle avant tout utilitaire. Un bel exemple dans la domus de la rue des Bouquets (Sablayrolles, 1988).

85. C'est par exemple le cas à Bibracte dans la Maison PC 1 où on constate l'apparition de l'hypocauste au moins dès 30 av. J.-C., associée à d'autres techniques romaines comme la maçonnerie, les sols en mortier, les tegulae et les imbrices (Paunier, Lunginbühl dir., 2004). Ces dernières sont employées dès le dernier quart du $\mathrm{II}^{\mathrm{e}} \mathrm{s}$. dans le centreest de la Gaule (Archéologia, 437, oct. 2006, p. 53).
La recherche de protection contre les rigueurs climatiques est caractéristique des maisons à péristyle où cet aménagement en a considérablement accru les effets négatifs, mais les réponses à ce problème varient souvent d'un cas à l'autre. Les manifestations les plus communes sont l'utilisation du verre à vitre 86 et, surtout, le chauffage d'un nombre plus ou moins élevé de pièces - non thermales - par hypocauste. On peut mettre sur le même plan l'usage préférentiel de la technique de construction à pan de bois et de terre, notamment pour les parties hautes ou l'étage et, dans certains cas, plus rares, l'obturation plus ou moins complète des entrecolonnements du péristyle (Vipard, 2001-2002, p. 39-56).

Une composante plus mentale cette fois, bien que servie par des aménagements matériels, semble discernable dans certaines domus: la recherche de la vue sur un paysage naturel ${ }^{87}$, voire urbain, pourrait avoir été plus fréquente qu'il n'apparaît dans les publications, mais bien évidemment ceci était fonction des possibilités topographiques locales. Cette préoccupation esthétique dont on commence à trouver des traces vers la fin de la République (Saliou, 1994, p. 232-247) et qui ne fait que croître sous l'Empire ${ }^{88}$, est intimement liée à la notion d'otium, comme les éléments de confort.

Ces diverses composantes secondaires, présentant de grandes variations de formes, n'existent souvent pas telles quelles dès la construction, mais peuvent évoluer, voire disparaître au cours de l'histoire de chaque maison; elles apparaissent plus comme des conséquences d'une adaptation à diverses situations que comme des éléments véritablement obligés de la maison romaine. Elles participent néanmoins au confort d'une demeure où tout semble d'ailleurs contribuer à créer une atmosphère paisible, du moins quand la maison est débarrassée de ses visiteurs et que le maître des lieux peut se livrer à l'otium.

86. Matériau nouveau et coûteux, le verre plat joue un rôle à la fois pratique et ostentatoire (voir par exemple Vipard, à paraître). Il ne devient courant qu'à partir de la seconde moitié du $\mathrm{I}^{\mathrm{er}}$ s., mais on en trouverait cependant des attestations extrêmement précoces (dès 30 av. J.-C.) dans la Maison PC 1 de Bibracte, par exemple (Paunier, Luginbühl dir., 2004, p. 82).

87. La Maison de la Plate-Forme à Fréjus semble avoir plus précisément eu vue sur le port; à Genève, celle de la Cour de la Prison SaintAntoine sur le lac Léman (Haldimann et al., 1991, p. 196) ; à Vienne, beaucoup de maisons ont une vue sur le Rhône ; celles de l'Insula 28, à Augst, ou du «Bas de Vieux », sur une vallée. Les cas doivent être nombreux, mais les publications ne permettent que rarement d'en prendre conscience.

88. Cette recherche d'une position dominante par rapport à un espace pourrait d'ailleurs être liée, outre à un caractère esthétique, au fait qu'elle crée une domination sociopolitique sur celui-ci (Drerup, 1959, p. 150). 


\section{LES DEMEURES ARISTOCRATIQUES DANS LA VILLE}

\section{DOMUS ET TOPOGRAPHIE}

On aura compris que la maison à péristyle, du fait de sa forte composante ostentatoire, n'est pas un édifice qui se suffit à lui-même : son emplacement, son rôle et l'efficacité de ce dernier dépendent de facteurs extérieurs qui la lient très étroitement à la ville où elle se dresse.

Dans les cités dont on connaît la topographie antique, la position près du centre est, de loin, la plus répandue. L'exemple le plus représentatif est sans conteste celui de Limoges avec sa concentration de maisons nobles dans les insulae voisines du forum (fig. 18). D'autres, pour ne pas être accolées au forum même, n'en sont cependant éloignées que de quelques centaines de mètres (c'est le cas de la Maison du Pinard à Alba-la-Romaine, par exemple) ou, bien qu'assez régulièrement dispersées dans toute la ville, n'en sont pas moins proches les unes des autres, comme à Arles où elles sont toutes regroupées dans un rayon d'à peine plus de $300 \mathrm{~m}$ (Collectif, 1996b, p. 10-11, fig. 1). On en connaît cependant un certain nombre qui sont nettement plus éloignées du centre-ville, sans qu'il s'agisse pour autant de villae suburbanae. Il s'agit généralement de demeures construites assez tardivement, vers la fin $d u \mathrm{I}^{\mathrm{er}} \mathrm{s}$. et surtout au $\mathrm{II}^{\mathrm{e}} \mathrm{s}$., sans doute parce qu'il n'était plus matériellement ou pas financièrement possible de trouver une place dans le centre, comme les domus du site du Palais des Sports à Amiens (Binet, 1996). Ce fait expliquerait l'aspect plus modeste de telles maisons. D'autres raisons permettant d'éclaircir ce fait nous échappent, comme dans le cas de certaines maisons de la ville basse à Saint-Bertrand-de-Comminges, alors que le centre paraît avoir été peu loti, voire vide de bâtiments par endroits (Paillet, Petit, 1992, p. 122). Parmi les cas de constructions périphériques, il faut faire une place à part aux maisons autrefois périurbaines rattrapées par l'urbanisation à la fin du $\mathrm{I}^{\mathrm{er}}$ s. ou durant le $\mathrm{II}^{\mathrm{e}}$ s., comme la Maison au Dauphin à Vaison-la-Romaine et celles de la Boissière à Jublains ou du Clos Mulon à Corseul. Le faubourg de Vienne sur la rive gauche du Rhône est un cas particulièrement remarquable d'habitat très riche apparemment assez éloigné du centre civique (fig. 19) ; ce dernier n'est toutefois pas dépourvu (notamment au nord) de maisons à péristyle (traces), mais la densité de constructions et le pendage du terrain y rendaient l'édification de très grandes domus presque impossible.

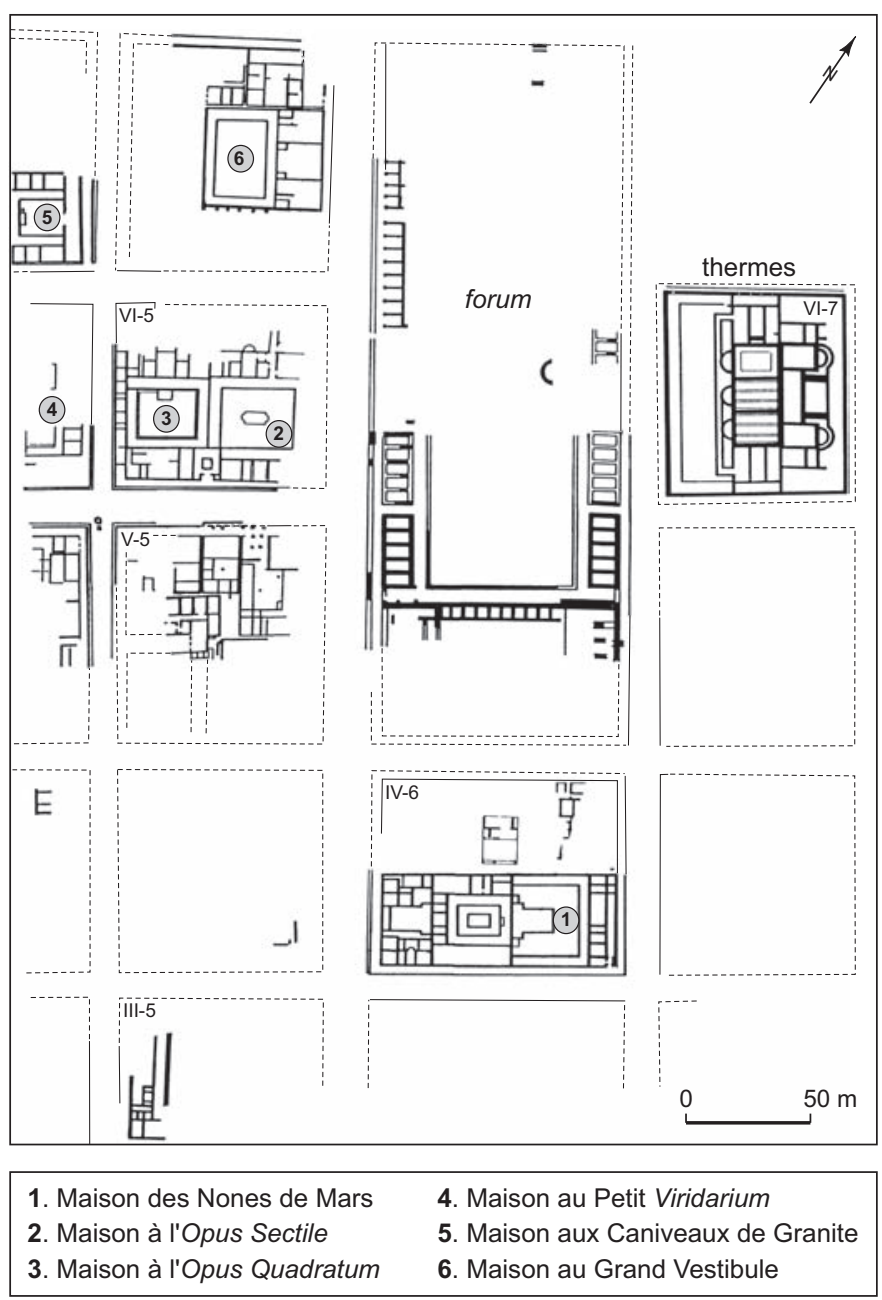

Fig. 18 - Des maisons au plus près du cœur civique : l'exemple $d u$ quartier $d u$ forum d'Augustoritum/Limoges (Haute-Vienne) (d'après Loustaud, 2000, fig. 26).

Du fait que la cité s'identifie avec les fonctions mêmes de l'État (Zaccaria Ruggiu, 1995, p. 31) et que le forum en est le centre, il ne faut sans doute pas chercher plus loin la raison de la fréquente position centrale d'un si grand nombre des plus riches domus à péristyle. L'intérêt de résider le plus près possible du forum permettait de faire rejaillir sur sa maison un peu de son prestige public et de montrer, par sa position, que l'on appartenait au pouvoir. Ce sentiment d'appartenance était d'ailleurs grandement favorisé par l'assimilation de ces maisons à des monuments publics par le biais de leur monumentalité et de l'utilisation de formes architecturales communes.

Cependant, les villes de Gaule étaient rarement si étendues que l'on ait dû avoir beaucoup à pâtir d'habiter dans un quartier légèrement périphérique, comme cela pouvait être 


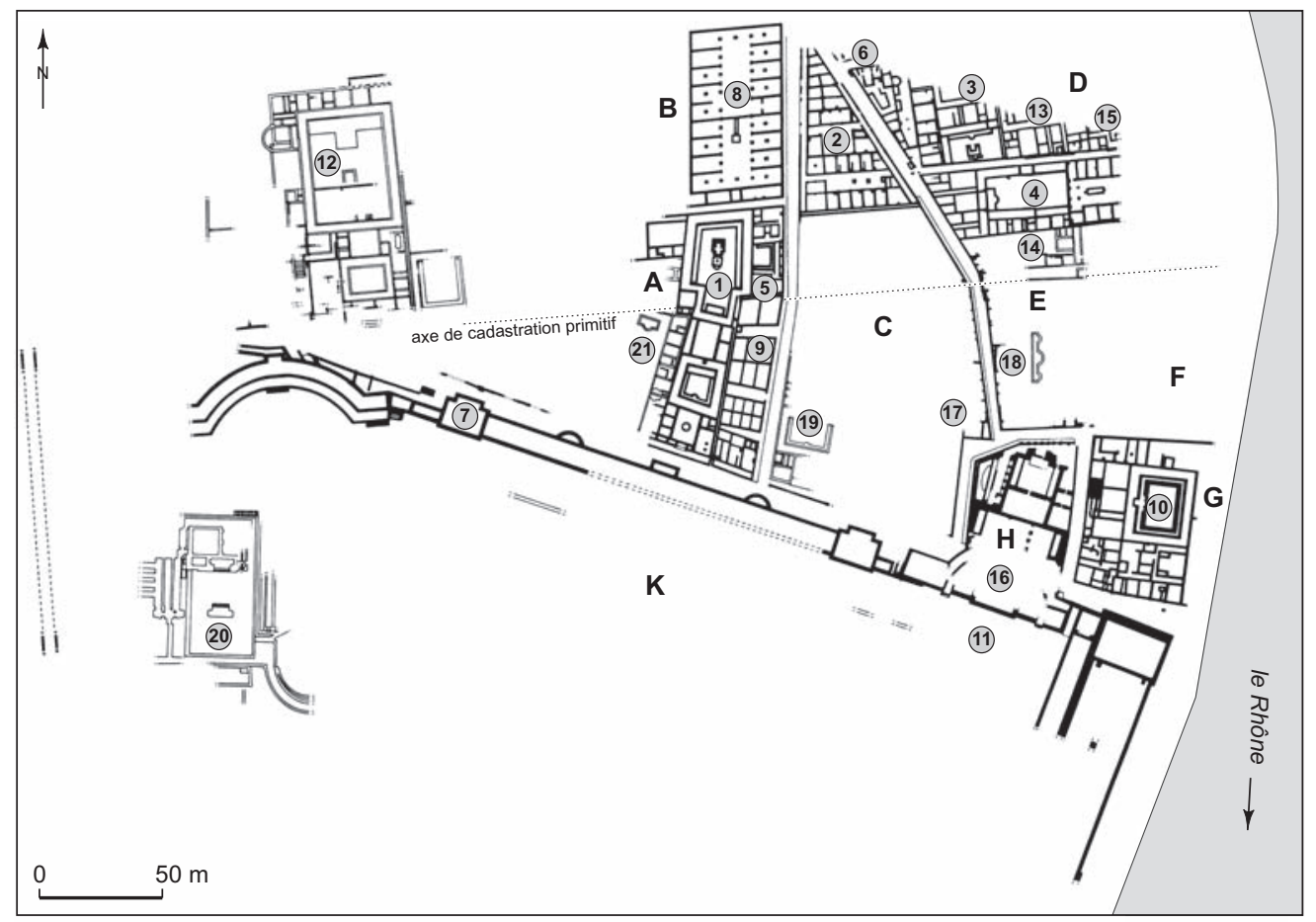

1. Maison des Dieux Océan

2. Artisanat et commerces (marché)

3. Maison aux Colonnes

4. Maison de Sucellus et édifice aux Piliers

5. Maison aux Cinq Mosaïques

6. Petits thermes du Nord

7. Portique nord et hémicycle

(ensemble monumental)

8. Grands entrepôts

9. Petits entrepôts

10. Maison au Lion

11. Portique du Rhône et esplanade centrale (ensemble monumental)

12. Maison A sous le Lycée

13. Maison aux Poissons

14. Maison

15. Maison anonyme

16. Thermes des Lutteurs (ensemble monumental)

17. Maison

18. Maison

19. Maison à la Colombe

20. Thermes du Palais du Miroir (ensemble monumental)

21. Maison

A à K. îlots

Fig. 19 - Plan du quartier de Colonia Julia Vienna/Vienne (Saint-Romain-en-Gal, Bouches-du-Rhône) sur la rive droite du Rhône, à la fin du II ${ }^{e}$ s. apr. J.-C. (d'après Collectif, 1996b, p. 354).

le cas dans certaines métropoles et que les clients aient eu l'occasion de le reprocher à un patron, comme le fait Martial (Épigrammes, I, 108) se plaignant à l'un des siens, Gallus, d'habiter une «belle maison certes, mais située au-delà du Tibre » («pulchra quidem verum transtiberiana domus »), l'obligeant ainsi à faire un long trajet pour aller le saluer.

Que la notabilité soit incompatible avec la résidence hors du centre est illustrée par un exemple littéraire du $\mathrm{II}^{\mathrm{e}}$ s. apr. J.-C., tiré de l'Âne d'or d'Apulée (I, 21), que l'on a déjà eu l'occasion de citer et qui possède sans doute une valeur universelle dans l'Empire. Il fournit une illustration très concrète de l'importance topographique de la maison pour évaluer le rang d'un individu : quand son héros Lucius arrive à Hypata, en Thessalie, et se renseigne sur Milon qu'il croit être l'un des premiers citoyens de la ville, la cabaretière qui connaît en fait bien le riche personnage « que son extrême avarice et sa bassesse sordide ont perdu de réputation », indique en riant qu'en fait « il habite en dehors de la cité et même hors de l'agglomération (littéralement: hors du pomerium) », ce qui fait penser à Lucius qu'il s'agit d'une plaisanterie.

Cette position centrale est très importante parce que l'ostentation ne se comprend que par rapport à un public, à une exposition à la vue de tous. Elle explique également le principe rarement enfreint de non-séparation d'avec un habitat modeste (tout comme la présence extrêmement fréquente de boutiques en façade). À cet égard, le cas gaulois le plus documenté et le plus représentatif est sans doute celui de Saint-Romain-en-Gal qui montre l'étroite imbrication des activités professionnelles (entrepôts, marché, foulonnerie...) et de nombreuses maisons à péristyle de grand luxe. L'éloignement très relatif de ce quartier de Vienne du centre civique (guère plus de $500 \mathrm{~m}$ ) pourrait d'ailleurs avoir été amplement compensé par la fréquentation élevée de ce quartier très actif.

Hormis de tels cas liés à une adaptation à des contextes locaux, l'occupation du centre urbain devait donc avoir une valeur plus symbolique que pratique. Dans les villes de Gaule, l'éloignement du centre influait sans doute sur la dignitas de son possesseur comme cela semble avoir été le cas à Rome ; cela explique pourquoi de grandes maisons s'y sont établies précocement, mais surtout pourquoi il semble y avoir eu une activité immobilière importante visant au regroupement de lots pour gagner assez de place, une fois cet espace déjà occupé par diverses constructions. Plus on avance dans le Haut-Empire, plus l'installation au cour de la cité peut donc être considérée comme une manifestation accrue de puissance. 
Ce lien organique très intime avec l'urbs peut donc être tenu pour une composante essentielle des domus à péristyle, même si les formes qu'elle peut revêtir varient bien évidemment pour chaque cas. Quand cela était possible, les maisons ne se sont pas contentées d'être situées au plus près du centre-ville, mais ont également tiré parti d'opportunités topographiques, voire d'inconvénients retournés au profit des aménageurs.

En Gaule, une manifestation originale de telles pratiques est constituée par les demeures sur terrasses que l'on rencontre dans la plupart des villes construites sur des reliefs marqués, mais dont les caractéristiques n'ont jamais été aussi bien mises en évidence qu'à Limoges où l'on a pu établir que ces plates-formes, quelquefois hautes de plus de $3 \mathrm{~m}$ et pour lesquelles il a fallu opérer d'énormes travaux de remblaiement (pas moins de $6500 \mathrm{~m}^{3}$ de roches et de gravats pour la seule maison des Nones de Mars), ont servi à l'ostentation de ces maisons en les plaçant en position dominante par rapport aux rues qui les bordaient et se trouvaient ainsi surplombées par de hauts murs aveugles en opus caementicium (Loustaud, 1996).

À l'inverse, il semble que l'on ait quelquefois tiré avantageusement parti d'une position défavorable (puisque dominée), comme la situation dans un quartier bas, pour exhiber aux yeux de tous les dimensions de sa maison. Ainsi, pour en revenir à l'exemple déjà cité de SaintRomain-en-Gal, à défaut d'un contact physique réel c'est un lien visuel qui pouvait s'établir entre le cœur de la ville haute, sur les pentes de la rive gauche du Rhône, et les maisons viennoises de la ville basse qui affichaient ainsi leur magnificence.

À côté de ces opportunités liées à la topographie des sites, on a plus généralement utilisé les possibilités de mise en valeur offertes par la proximité d'un monument public ou par la disposition de la trame viaire. Dans le premier cas, ce peut être l'accolement à un monument prestigieux faisant rejaillir son prestige sur la maison, comme pour la Maison de la rue des Bouquets située à côté du grand temple à Périgueux (qui a lui-même recouvert une domus à péristyle), face au forum et à sa basilique ou, à Saint-Romainen-Gal, le voisinage de la Maison au Lion avec les thermes des Lutteurs ce qui met en valeur le gigantisme de la première. Dans le second cas, par exemple, on a utilisé une voie pour focaliser le regard sur l'entrée monumentale de la domus, comme dans le cas de la Maison au Grand Péristyle à Vieux (fig. 7).

Cependant, les liens avec la ville ne sont pas seulement d'ordre matériel et utilitaire.

\section{LA PLACE DES DOMUS ARISTOCRATIQUES DANS LA VILLE}

Au même titre qu'un quelconque monument public, les maisons, surtout riches, sont un repère topographique, un toponyme (elles avaient en effet un nom), mais sont aussi et avant tout un élément reconnu de la parure urbaine, d'où leur importance considérable dans l'espace urbain, qu'elles soient isolées ou groupées, en fonction du nombre de primores civitatis capables d'en posséder une.

L'association de la notion de pulchritudo urbis à celle de la dignitas et de la majestas de l'État est un lieu commun depuis Cicéron qui a perduré sous l'Empire. Dans son programme politique, la primauté a en effet été accordée par Auguste à la civitas avec, notamment, dans les provinces conquises par la force, l'intention de donner un fondement au crédit politique et moral de l'image de la cité en jouant sur le prestige esthétique, fonctionnel et représentatif des édifices publics (Zaccaria Ruggiu, 1995, p. 199-228 et 1996).

On peut donc supposer que la proximité des maisons nobles du centre-ville trouve une autre justification dans le fait que leur beauté, par ses renvois manifestes aux formes de l'édilité publique, concourt elle aussi à la majestas imperii. Cet aspect n'est pas explicitement inscrit dans les textes, mais est amplement confirmé par un ensemble de lois concernant souvent spécifiquement les maisons et visant à empêcher leur dépouillement, leur ruine ou leur destruction, qui constitueraient un acte pouvant contredire la majestas du peuple romain.

Ces mesures législatives existent dès le $\mathrm{I}^{\mathrm{er}} \mathrm{s}$. av. J.-C. dans des lois municipales, mais c'est seulement à partir de Claude et régulièrement à travers des rescrits impériaux tout au long de l'Empire ${ }^{89}$ qu'elles expriment clairement, derrière la condamnation de la spéculation, leur intention principale résumée par le recours à une expression récurrente : ne ruinis urbs deformetur («afin que la ville ne soit pas défigurée par les ruines »).

L'histoire des maisons à péristyle (de Gaule notamment) est beaucoup plus tributaire qu'on le pense de cette législation universelle ${ }^{90}$, même si elles ne sont bien évidemment jamais désignées explicitement: jusqu'en 212, en effet, ces

89. On peut trouver un récapitulatif sommaire, mais très commode, dans Zaccaria Ruggiu, 1995, p. 225-228, spécialement dans les textes $\mathrm{n}^{\text {os }} 7-14,16,20,21,23,24,25$ (pour le Haut-Empire) ; voir aussi Garnsey, 1976.

90. Au début du III $^{\mathrm{e}}$ s., le jurisconsulte Ulpien indique en effet qu'elle s'entend pour toutes les villes, et non seulement pour Rome (Digeste, XXX, 41, 9). 


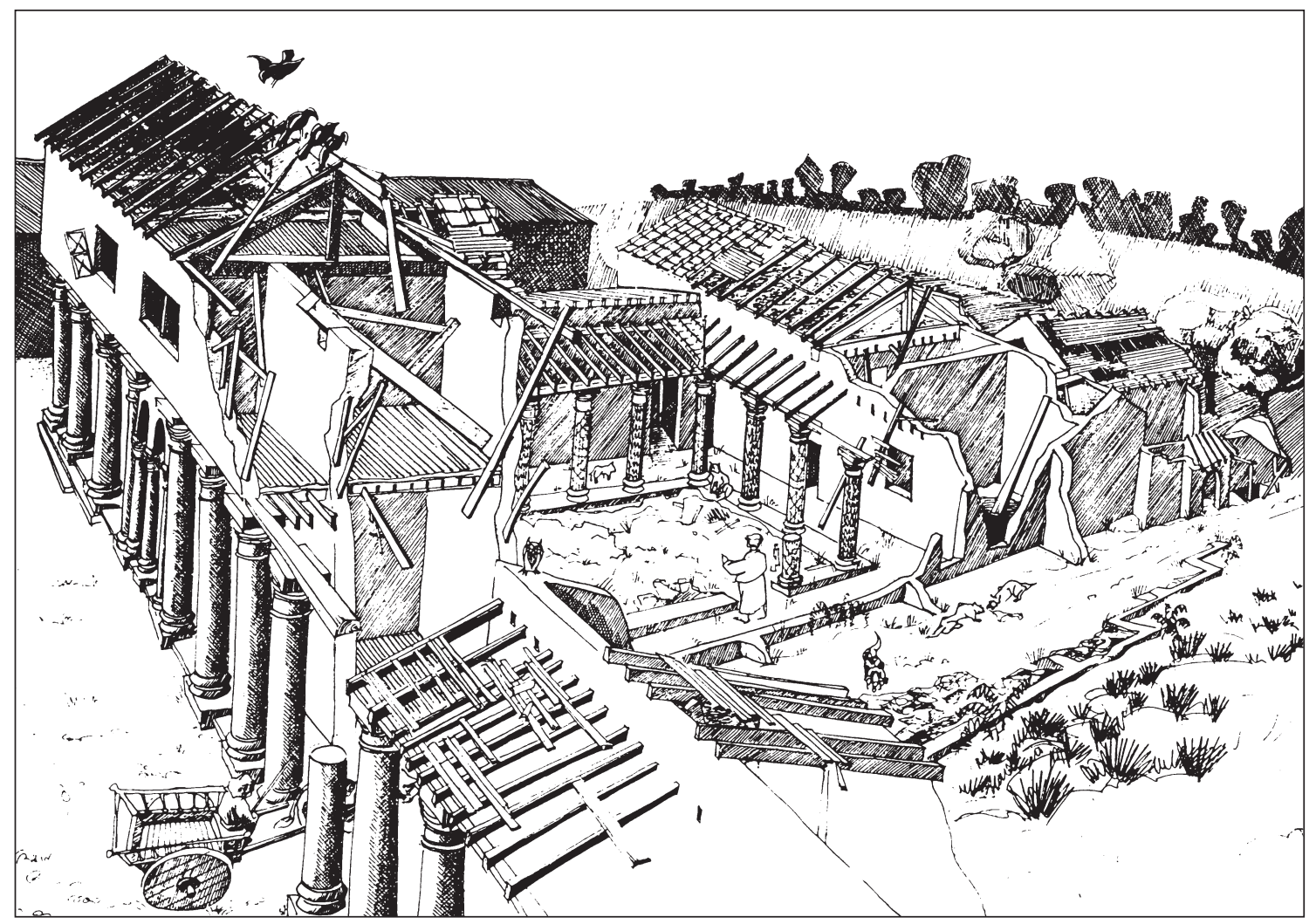

Fig. 20 - Reconstitution des ruines de la Maison du «Bas de Vieux» (Calvados) percées par une voie au deuxième quart du IV s. (dessin: L. Verrier, architecte DPLG et P. Vipard, Université Nancy-II).

lois s'appliquent en principe aux seuls citoyens et, comme dans la plupart des cités de Gaule, ceux-ci sont presque exclusivement les notables, leurs maisons sont donc principalement concernées. Étant les bâtiments les plus riches et morphologiquement les plus proches des monuments publics, les domus sont de toute façon plus concernées par la loi que les constructions modestes ; ceci ressort clairement des rares détails matériels expressément cités concernant la décoration : marbres et colonnes, ce dernier élément ne laissant en effet aucun doute sur le type de maison désigné. Toutes ces mesures ont pour but d'empêcher la dégradation ou la destruction de maisons qui nuiraient à l'esthétique de la ville en obligeant les propriétaires (souvent les héritiers) à les entretenir ou à les préserver. Les cas où une grande maison à péristyle est détruite par son propriétaire pour édifier à la place un édifice public n'entre bien évidemment pas dans le cadre de ces lois ${ }^{91}$.

91. L'archéologie n'est cependant généralement pas en mesure de déterminer si l'on est en présence d'un tel acte d'évergésie ou d'une opération immobilière menée par les édiles. La première hypothèse est probable dans le cas du grand sanctuaire de Périgueux (Tour
Cette législation pour la protection du paysage urbain laisse également entrapercevoir d'autres enjeux: par exemple le rôle de ces maisons dans l'émulation entre cités, notamment avec l'interdiction par Hadrien de transporter des matériaux d'une cité à une autre ${ }^{92}$. Les quelques exceptions à ces interdictions de destruction sont également très significatives : la principale, le sénatus-consulte Acilianum de 122 apr. J.-C. ${ }^{93}$, lève ainsi l'interdiction de legs testamentaire du matériel décoratif des maisons, dans le seul cas où celui-ci est destiné à des ouvrages publics. Ce cas de remploi autorisé l'est donc uniquement parce qu'il ne

de Vésonne) au cours du II $^{\mathrm{e}}$ s. (Girardy-Caillat, 1992, p. 126-127 et fig. 50) ; en revanche, on ne peut le savoir dans le cas de la Maison de l'Insula XVI à Leicester, ruinée à la fin du $\mathrm{II}^{\mathrm{e}} \mathrm{s}$. et remplacée par un marché public au début du $\mathrm{III}^{\mathrm{e}} \mathrm{s}$. (Wacher, 1981, p. 348-350).

92. Voir SHA, Vita Hadriani, 18, 2 ; cet édit semble être une précision à l'édit de Vespasien interdisant de transporter des matériaux de récupération (marbre et matériaux de construction) d'une maison à une autre, sans préciser si c'était dans la même cité ou à l'extérieur. Cette interdiction est réitérée par Ulpien (Digeste, XXX, 41, 5).

93. Repris dans un rescrit de Septime Sévère et de Caracalla rappelé par Ulpien (Digeste, XXX, 41, 5). 
soustrait rien à l'ornement de la ville. Il montre également comment des propriétaires n'ont guère d'autre choix que de se conduire en évergètes en offrant colonnes ou plaques de marbre précédemment utilisées dans des bâtiments privés. Ce cas administre d'ailleurs une nouvelle preuve du caractère public de l'architecture et du décor domestiques riches puisque qu'ils peuvent indifféremment, et successivement, orner aussi bien un bâtiment domestique que public. L'autre exception à la règle est celle de l'autorisation de transformation d'une maison en jardin ${ }^{94}$, ce qui implique un démontage, un nivellement et une transformation faisant certes disparaître une construction, mais surtout ses ruines, au profit d'un espace qui, au final, ne défigure pas la cité. Ce pis-aller, sans doute le plus économique - mais qui reste néanmoins une évergésie ${ }^{95}$-, permettrait peut-être d'expliquer un certain nombre d'exemples gaulois de maisons dépourvues d'épais niveaux de destruction et où l'on ne constate absolument plus de traces d'occupation avant des siècles. Hormis cette dernière possibilité, il faut reconnaître qu'il est presque impossible de mettre concrètement en évidence la réalité matérielle de l'application de ces lois de protection du paysage urbain. Un cas un peu tardif de cette politique de préservation de l'integritas urbaine et qui concerne une domus à péristyle déjà en grande partie en ruine est fourni par la Maison du «Bas de Vieux » (fig. 20) : dans le premier tiers $d u \mathrm{IV}^{\mathrm{e}} \mathrm{s}$., celle-ci est en effet traversée par une voie publique qui détruit toute son aile occidentale, mais laisse en place les colonnes de la façade (au prix d'un léger contournement de la chaussée à cet endroit).

Ces lois sont édictées dès le $\mathrm{I}^{\mathrm{er}} \mathrm{s}$., mais leur fréquente réitération dans le premier tiers $d u \mathrm{III}^{\mathrm{e}} \mathrm{s}$. est sans doute significative d'un accroissement du problème à l'époque sévérienne. Cette constatation conduit à parler brièvement de la désaffection et de la disparition des maisons à péristyle. Brièvement, parce qu'il faut encore une fois constater que les données écrites spécifiquement gauloises manquent et que l'archéologie n'y supplée guère, souvent faute d'intérêt de la part des fouilleurs, mais plus souvent encore faute d'indices pertinents.

94. Code Justinien, VIII, 10, 3 (Sévère Alexandre, 224 apr. J.-C.) qui précise que, dans les chefs-lieux de cité, cette transformation de maisons déjà en ruines doit se faire avec l'accord des magistrats, mais que pour les plus éminentes le gouverneur de la province doit faire une enquête.

95. À la même époque, à Rome, Sévère Alexandre crée, pour agrémenter ses thermes, un parc boisé sur un terrain précédemment occupé par des maisons qu'il avait achetées pour les faire démolir ( $S H A$, Alexander Severus, XXV, 4).

\section{LA FIN DE LA DOMUS À PÉRISTYLE EN GAULE}

En Gaule, contrairement à ce que l'on observe en Orient (Saliou, 1990) ou en Afrique (Thébert, 1985), où les maisons urbaines à péristyle connaissent encore de beaux jours durant l'Antiquité tardive, la domination sociale de cette forme d'habitat a connu une vie assez brève, très liée à la romanisation de la société et à l'urbanisation, et disparaissant avec la perte de prépondérance de celle-ci au BasEmpire.

Le phénomène a jusqu'alors été peu étudié ${ }^{96}$ et il est vrai que la documentation disponible est encore une fois très lacunaire. Même pour les fouilles récentes, les archéologues sont généralement peu soucieux de cet aspect qui demande une attention très particulière; plus souvent, il est vrai, les conditions de fouilles ou la rareté, voire l'absence, d'indices constituent des obstacles insurmontables à une telle étude.

Les conditions et les processus d'abandon de ces maisons sont donc assez mal connus et sont en outre compliqués par le fait que les publications ne permettent pas toujours de faire la distinction entre un abandon et une destruction, dont les significations respectives ne sont bien évidemment pas les mêmes. Tout en étant conscient des limites de nos connaissances, il n'est pas inutile d'en dresser un bilan sommaire.

On note quelques abandons ou destructions assez précoces, dès le dernier tiers $\mathrm{du} \mathrm{I}^{\mathrm{er}} \mathrm{s}$. ou au début du $\mathrm{II}^{\mathrm{e}}$ s. ${ }^{97}$. Ce sont des maisons généralement petites et/ou situées dans une zone excentrée de la ville, ce qui pourrait expliquer qu'elles n'ont pas été reconstruites; ce pourrait être encore le cas de la Maison du site de «Ma Maison " à Saintes, abandonnée vers le milieu du $\mathrm{II}^{\mathrm{e}} \mathrm{s}$. (Maurin dir., 1988, p. 30-31).

Un certain nombre de cas de destructions, non suivies de reconstructions, est attesté à la fin du $\mathrm{II}^{\mathrm{e}} \mathrm{s}$. ou au tout début du III ${ }^{\mathrm{e}}$ s. ${ }^{98}$. À partir du milieu du III ${ }^{\mathrm{e}}$ s. et dans la seconde

96. Ce problème, quand il est abordé, est généralement évacué en quelques mots. Pour les grandes lignes du problème dans l'Empire (mais ne s'adaptant pas toujours parfaitement à la Gaule), voir Ellis, 1988.

97. La Maison aux Xenia à Lyon (démolie au début des années 70), peut-être la maison rue de la Fraternité à Arras (détruite par le feu à une date imprécise au $\mathrm{II}^{\mathrm{e}} \mathrm{s}$.), le groupe des maisons nîmoises 8, 10, 12, 14 et 9 de la ZAC Villa Roma (au tournant des I $^{\text {er }}$ s. et II $^{\mathrm{e}}$ s. ; vers 120-140 pour le dernier cas) et A et B du site des Villégiales (milieu du $\mathrm{II}^{\mathrm{e}} \mathrm{s}$.).

98. La Maison du Clos de la Lombarde (état 2), celle de l'Insula XVI à Leicester, les deux domus du Terrain Peigné à Rezé, la Maison de Berkeley Street à Gloucester (démolition), celle du 18 rue des Magnans à Aix-en-Provence (début du III ${ }^{\mathrm{e}} \mathrm{s}$. ?), celle de la Cour d'Albane à 
moitié de ce siècle ${ }^{99}$, plus encore dans le troisième quart ${ }^{100}$ et au tournant du IV ${ }^{\mathrm{e}}$ s. ${ }^{101}$, les cessations d'occupation ou les destructions s'accélèrent. Au IV ${ }^{\mathrm{e}}$ s., paradoxalement, sans cesser, les cas de disparition sont moins nombreux ${ }^{102}$ et un peu plus fréquents vers la fin du siècle ${ }^{103}$.

Quoique inéluctable, la tendance à la disparition connaît des résistances en Gaule. On connaît ainsi quelques cas de construction tardifs en Gaule Belgique et en Lyonnaise, à Amiens ${ }^{104}$ et à Carhaix-Plouguer ${ }^{105}$ au début du IV ${ }^{\mathrm{e}} \mathrm{s}$. (disparition dans la seconde moitié du $\mathrm{IV}^{\mathrm{e}} \mathrm{s}$.). Bien que très sommairement documentés pour l'instant, ces exemples, qui semblent avoir eu une courte durée de vie, pourraient éventuellement constituer une manifestation du fugace sursaut urbain de l'époque tétrarchique lié au rétablissement de la puissance de Rome dans l'Empire et au retour à

Rouen (incendie du quartier dans les premières décennies du $\operatorname{III}^{\mathrm{e}} \mathrm{s}$.), et enfin celle de l'Atrium II à Vienne (première moitié du III ${ }^{\mathrm{e}} \mathrm{s}$.).

99. La Maison 3 (état 2) des Chartreux à Aix-en-Provence, celle au Dauphin à Vaison-la-Romaine (destruction), celle sous les Thermes impériaux (Insula nord) à Trèves, la Maison E1 (état II) de SaintFlorent à Orange, celle des Dieux Océan (seconde moitié du III $^{\mathrm{e}} \mathrm{s}$. ?), celle aux Cinq Mosaïques à Vienne (?), celle du Clos Mulon à Corseul (écroulement des ruines), et enfin celle aux Colonnes (état 2) à Vienne.

100. Les trois Maisons de la rue de la Harpe à Évreux (incendie du quartier vers 275-285), celle du Crédit Agricole à Arles (destruction), celles à la Mosaïque aux Méandres et à la Mosaïque Polychrome du quartier Saint-Florent à Orange, celle de l'Aire du Chapitre à Aix-enProvence (abandon, récupération des matériaux), la Maison A des Basaltes à Alba-la-Romaine (abandon), celle de la rue des Bouquets à Périgueux, enfin celle à l'Enseigne à Autun (abandon).

101. Maison de l'Îlot B de la place Camille-Jouffret à Vienne (destruction) ; celle du «Bas de Vieux » à Vieux (destruction et démontage) ; celle des Nones de Mars à Limoges (abandon et démontage) (toutes les trois à la fin du $\mathrm{III}^{\mathrm{e}} \mathrm{s}$.-début du $\mathrm{IV}^{\mathrm{e}} \mathrm{s}$.) ; celle du Dieu Marin à Aixen-Provence, Enclos Milhaud (III ${ }^{\mathrm{e}}-\mathrm{IV}^{\mathrm{e}} \mathrm{s}$.) ; celle de la Place Formigé (état 2) à Fréjus (première moitié du $\mathrm{IV}^{\mathrm{e}} \mathrm{s}$.).

102. Au milieu du IV ${ }^{\mathrm{e}} \mathrm{s}$.: Maison de la Fontaine des Bénédictins à Nîmes (abandon), de l'Îlot Neyron à Clermont-Ferrand (abandon après incendie) ; les Maisons de Nyon et de Martigny (IV ${ }^{\mathrm{e}}$ s., sans plus de précision).

103. Maison de Balbius Iassus à Autun (destruction), celle du Génie Privé (Insula 8) à Martigny (abandon), celle de l'Insula III à Verulamium/Saint-Albans (abandon), les Maisons IIa, IIb et IIIa de l'Insula III du site du Clos de la Tour à Fréjus, enfin celles du Jardin de Grassi : au Péristyle Rhodien, au Bassin à Absides, au Grand Péristyle $\left(\right.$ IV $^{\mathrm{e}}-\mathrm{V}^{\mathrm{e}} \mathrm{s}$. ?).

104. Site du Bas Parvis de la Place Notre-Dame (fouille A. Dubois) : une domus à péristyle, datée des $\mathrm{III}^{\mathrm{e}}-\mathrm{IV}^{\mathrm{e}} \mathrm{s}$., est construite sur deux demeures du III ${ }^{\mathrm{e}}$ s., partiellement observées (Dubois, in Cerruti-Déroff dir., 1996, p. 21).

105. Vorgium/Carhaix-Plouguer (Finistère), Centre hospitalier (fouille G. Le Cloirec) : un des îlots est entièrement restructuré au $\mathrm{IV}^{\mathrm{e}} \mathrm{s}$. apr. J.-C. pour accueillir une domus, de construction soignée, qui en occupe toute la surface, soit plus de $2500 \mathrm{~m}^{2}$; l'ensemble est réoccupé sommairement et partiellement démonté dans la seconde moitié du $\mathrm{IV}^{\mathrm{e}}$ s. (Collectif, 1997, p. 16 et p. 37-38). une sécurité relative (qui se concrétise notamment dans la région de la Manche et de la mer du Nord, par le début de la mise en place du Litus Saxonicum).

D'autres cas sont connus, dans le sud-ouest de l'Aquitaine qui se distingue nettement du reste de la Gaule à cette époque. C'est là, par exemple, que l'on rencontre les seules attestations assurées d'une poursuite de l'occupation des maisons du Haut-Empire jusqu'à une date avancée de l'Antiquité tardive ${ }^{106}$; le seul vraiment assuré est celui de la modeste Maison à Atrium de Saint-Christoly à Bordeaux, occupée sans hiatus du $\mathrm{II}^{\mathrm{e}} \mathrm{s}$. aux V $\mathrm{V}^{\mathrm{e}}-\mathrm{VI}^{\mathrm{e}} \mathrm{s}$. (Balmelle, 1996, fig. 4.1, p. 124 et p. 126). C'est également dans cette région que l'on note, au début $d u \mathrm{IV}^{\mathrm{e}} \mathrm{s}$., après une courte période d'abandon, le cas exceptionnel de reconstruction d'une maison à péristyle sur le même plan que le précédent : la Maison de la rue des Bouquets à Périgueux (Coupry, 1975, p. 472-473 et fig. 17) et, vers le milieu du IV ${ }^{\mathrm{e}}$ s., celui d'une construction nouvelle de maison à péristyle en milieu urbain, à Eauze, dans le faubourg occidental (CAG, 32, p. 145). Ces exemples aquitains peuvent être rattachés à la période de paix régnant dans le Sud-Ouest au $\mathrm{IV}^{\mathrm{e}} \mathrm{s}$. et à la forte imprégnation de romanité de ses élites jusqu'au VII ${ }^{\mathrm{e}} \mathrm{s}$. (Balmelle, 2001, p. 23-53). Il faut toutefois remarquer que les maisons à péristyle connues auparavant semblent généralement détruites ou ne connaissent pas de pose de ces somptueuses mosaïques si caractéristiques de l'habitat des notables du Bas-Empire dans la région.

Les dates d'abandon ou de destruction des maisons à péristyle montrent que leur disparition ne semble pas avoir obéi à une décision brusque et uniforme. Ce qui est certain, c'est que l'on ne possède pas, sauf exceptions citées ci-dessus, de traces de constructions nouvelles ou même de transformations notables après l'époque sévérienne. De même, les campagnes de décoration somptuaire, très dynamiques à cette époque, s'arrêtent presque totalement (ou, du moins nous n'en avons pas identifié les traces). Après le premier quart (ou peut-être le premier tiers) du $\mathrm{III}^{\mathrm{e}} \mathrm{s}$., il semble que l'on se soit, partout en Gaule, installé dans de l'acquis. Plus que l'atteinte d'un équilibre entre le nombre de membres des élites et celui des demeures aristocratiques, il semble que l'on trouve dans ce constat la traduction de la dégradation de la situation économique et sociale contemporaine. Dès l'époque sévérienne, le fossé s'est sans doute creusé entre les notables capables ou non d'assurer l'entretien et le fonction-

106. Ailleurs les cas sont très incertains : le Jardin de Grassi à Aixen-Provence (Benoît, 1947) est très imprécisément relaté et les traces d'occupation du haut Moyen Âge de la Maison de la Boissière à Jublains semblent plutôt liées à une réoccupation (CAG, 53, p. 63-65). 
nement d'une telle maison, de plus le nombre des premiers s'est considérablement réduit, ce qui expliquerait l'arrêt des nouvelles constructions et les premières vagues importantes de disparitions. C'est sans doute dans cette situation de crise économique qu'apparaît clairement la prépondérance du rôle ostentatoire du péristyle (grand consommateur de place et de décor), et donc point principal des dépenses (d'aménagement et d'entretien), qui paraît en fait être une cause majeure de la désaffection des maisons nobles où il est quelquefois le premier élément à disparaître. La législation contemporaine enregistre d'ailleurs cette « inutilité ». Le jurisconsulte Paul (époque sévérienne) cite en effet une exception au sénatus-consulte Acilianum (Digeste, XVIII, 1, 21, 2) : un héritier peut enlever d'une habitation colonnes et poutres (trabes) à condition de ne pas entraîner la destruction de ladite maison. Il est évident que, vu cette dernière restriction, les trabes en question ne peuvent être que celles des portiques.

Les premières traces de la désaffection pour les maisons à péristyle pourraient d'ailleurs avoir commencé à se faire jour alors même qu'elles connaissaient un floruit somptuaire à la fin de la période des Antonins et sous les Sévères. Dès la fin du $\mathrm{II}^{\mathrm{e}}$ s., l'archéologie montre en effet que certaines domus pourraient déjà s'être écroulées par abandon et qu'en cas d'incendie accidentel, on n'aurait pas jugé bon de les reconstruire (malgré la législation interdisant ces pratiques). Sur ce point, l'exception constituée par la Maison du Clos de la Lombarde est peut-être très révélatrice, puisque après sa destruction par le feu à la fin du $\mathrm{II}^{\mathrm{e}} \mathrm{s}$., elle est cependant reconstruite et très richement redécorée, à l'exclusion de sa moitié occidentale autrefois occupée par le péristyle, et est laissée à l'abandon jusqu'à la fin de l'occupation de la nouvelle maison vers 210-230. Il est toutefois toujours difficile d'interpréter des cas peu nombreux.

En revanche, statistiquement, l'accroissement des occurrences confirme le phénomène d'abandon au fur et à mesure que l'on avance dans le III $^{\mathrm{e}}$ s., mais les assez nombreux exemples d'occupation de maisons au IV ${ }^{\mathrm{e}} \mathrm{s}$. font illusion. Les cas documentés montrent en effet que lorsqu'il y a occupation des maisons à péristyle, celle-ci est le fait de gens d'un niveau social différent de celui des occupants précédents. Parce qu'on ne sait rien du statut juridique des maisons ou de leurs légitimes propriétaires à ce moment-là, on se gardera donc de parler de squatteurs pour désigner les occupants dont on observe les traces archéologiques et qui n'ont sans doute pas toujours mérité ce nom. Bien que ce ne soit pas typique des seules maisons à péristyle, leurs bâtiments, ruinés ou non, ont fréquemment été occupés, souvent après un hiatus dans l'occupation, par des habitants exerçant des activités artisanales souvent liées aux arts du feu (et qui ont donc fréquemment causé l'incendie des maisons en question) ${ }^{107}$. Actuellement, il n'y a pas de preuve indéniable de la présence des élites dans les maisons à péristyle à partir du milieu du III ${ }^{\mathrm{e}}$ s. ${ }^{108}$. Cela est peut-être dû à l'absence de travaux somptuaires et de marqueurs archéologiques pour cette période; cependant quelques cas montrent la présence d'une occupation d'une relative bonne qualité sociale jusqu'à une date avancée du $\mathrm{III}^{\mathrm{e}}$ s., mais l'impression générale est celle d'une très nette diminution sociale des occupants. De plus, à partir des années 260 notamment, il ne faut sans doute pas minimiser l'importance du danger que la possession de telles maisons faisait encourir à leurs propriétaires en les désignant à la convoitise des pillards germaniques ou des militaires ${ }^{109}$.

Même si toutes les destructions de la seconde moitié du $\mathrm{III}^{\mathrm{e}} \mathrm{s}$. ne sont sans doute pas à mettre systématiquement au compte des invasions, elles montrent qu'à cette date leurs propriétaires n'habitent vraisemblablement plus ce type de maisons puisqu'elles ne sont jamais reconstruites, ce qui revient à dire que les élites urbaines n'habitent plus en ville.

Sa disparition est en fait liée à celles des notables. En esquissant la situation du Bas-Empire, les réformes de Septime Sévère concernant le caractère désormais régulier et obligatoire des évergésies, l'obligation d'avancer la somme des impôts de plus en plus difficile à lever et d'entretenir les édifices publics ainsi que l'impossibilité d'échapper aux charges, commencent à rendre moins attractive la recherche

107. Pour ne prendre que quelques exemples, des activités sidérurgiques ont été repérées dans la Maison de la rue des Bouquets à Périgueux (Coupry, 1975, p. 473), dans la Maison nord du Terrain Peigné (qui abritait également un potier) à Rezé (Deschamps et al., 1992, p. 123), dans l'Îlot D du site de l'Arsenal, à Metz, qui hébergeait également des bronziers, bronziers que l'on trouve dans la maison de l'Insula 12E d'Avenches ou dans la Maison A du site de l'Ancien Évêché de Poitiers, associée à des tabletiers (Le Masne de Clermont, 1987, p. 157-158), eux-mêmes également attestés à Autun, dans la pièce $\mathrm{H}$ de la Maison de Balbius Iassus (Blanchard-Lemée et al., 1986, p. 148). Signalons enfin un verrier dans la Maison de la rue des Colonnes à Vienne (Lasfargues, 1982, p. 403).

108. À Limoges, dans la Maison des Nones de Mars, l'occupation par des membres de l'élite est encore attestée en 235 par un graffiti faisant apparaître une date consulaire (Bost, 1993, p. 54) : en outre, d'importants travaux somptuaires (notamment un opus sectile et des lambris de marbre, la réalisation d'une abside dans la salle S6) y sont datés postérieurement à 226-235 (Loustaud, 2000, p. 199). À Vieux, dans la seconde moitié du III $^{\mathrm{e}} \mathrm{s}$., malgré le maintien d'un niveau de vie relativement élevé dans la Maison à la Mosaïque à Damier, qui prend la suite de la Maison au Grand Péristyle, les bassins du viridarium et l'adduction d'eau courante disparaissent (Vipard, 2002, p. 90).

109. Par exemple, les troupes de Victorin détruisent des maisons à Autun en 269 (Panégyriques latins, IV, 4). 
des honneurs. Ainsi, dans nombre de cités, devant le peu de candidats, l'ostentation devient inutile pour s'imposer. De fait, archéologiquement et épigraphiquement, la vie municipale de la fin de l'époque sévérienne ne montre guère de dynamisme spontané.

À partir du moment où les élites quittent les maisons à péristyle, celles-ci ne sont alors plus que des bâtiments comme les autres, dans lequel le péristyle est sans doute plus une gêne qu'un avantage. En Gaule toutefois, comme la place ne manquait guère dans les villes dépeuplées, ce dernier semble avoir rarement subi ces cloisonnements si courants dans d'autres parties de l'Empire ${ }^{110}$. En ville, hormis dans quelques exemples aquitains, la maison à péristyle disparaît donc du paysage urbain, socialement vers le milieu ou la seconde moitié du $\mathrm{III}^{\mathrm{e}}$ s., physiquement surtout dans la seconde moitié et à la fin du $\mathrm{III}^{\mathrm{e}} \mathrm{s}$. et au $\mathrm{IV}^{\mathrm{e}} \mathrm{s}$., mais continue à connaître un grand succès dans les villae rurales où - ce n'est pas un hasard -, ces mêmes élites qui résidaient autrefois en ville ont reporté leur activité sociale au cours de la seconde moitié du III $^{\mathrm{e}} \mathrm{s}$.

La disparition des maisons à péristyle était inscrite dans les changements socio-économiques du $\mathrm{III}^{\mathrm{e}} \mathrm{s}$. et dans le déclin urbain de la majeure partie des villes gauloises. Malgré l'accroissement des décrets de protection de leur intégrité physique au début du $\mathrm{III}^{\mathrm{e}} \mathrm{s}$., l'augmentation de leur fréquence à l'époque tétrarchique et après montre leur inefficacité et l'incapacité des autorités impériales ou municipales, devenues propriétaires des biens abandonnés ou tombés en déshérence, à faire appliquer cette législation. D'une manière propre à la Gaule, le problème des domus à péristyle a donc trouvé une solution dans la réduction des cités derrière leurs murailles entre la fin du $\mathrm{III}^{\mathrm{e}} \mathrm{s}$. et le début $\mathrm{du} \mathrm{V}^{\mathrm{e}} \mathrm{s}$, et la plupart des maisons à péristyle ont fini par s'écrouler d'elles-mêmes ou, plus souvent encore, sous l'effet des récupérateurs de matériaux.

$$
\text { * } *
$$

Tous les problèmes posés par la nature des maisons à péristyle, dont la richesse informative a longtemps été sinon négligée du moins sous-exploitée, sont loin d'être levés, mais il semble acquis qu'elles ne correspondent pas à un genre artificiel créé par le désir de catégorisation de notre moderne système de pensée occidental. Le choix

110. On en signale un cas à Limoges dans la Maison des Nones de Mars, dans le portique du viridarium de l'aile nord, divisé en une succession de petites salles séparées par des murs posés à même le sol bétonné et dont les entrecolonnements ont été obturés par des matériaux de remploi (Loustaud, 1992, p. 73). d'un angle d'étude plus social que celui purement architectural ou archéologique qui a longtemps prévalu n'éclaire bien évidemment qu'un des multiples aspects de ce type d'habitat au sein duquel les fonctions domestiques sont finalement secondaires. Il permet cependant de mieux comprendre et de mesurer la place capitale occupée par lui dans la société et dans les cités gauloises. La maison urbaine que l'on retrouve en Gaule est fondamentalement celle élaborée sous la République à Rome par et pour les sénateurs de la Ville, et dont la forme que l'on va connaître sous l'Empire a été en quelque sorte fixée par Auguste ${ }^{111}$. Le modèle est alors mis au service d'un système politicoadministratif adapté à l'exercice du pouvoir - surtout par le biais de la pratique clientélaire et de la promotion individuelle - des membres des sénats locaux, rouage essentiel du système administratif mis en place dans le cadre urbain à travers lequel Auguste a souhaité organiser la Gaule.

Les aspects architecturaux ou matériels restent importants parce qu'ils sont les premiers décelables par l'archéologie et que c'est essentiellement à travers eux que l'on est ensuite capable de comprendre une partie des multiples rôles joués par ces maisons qui se définissent avant tout par des manifestations du negotium. Des caractéristiques matérielles, certes contraignantes mais organisées de façon assez souple en fonction de paramètres très variables (selon le principe très romain de l'adaptatio), sont cependant inextricablement mêlées à des composantes plus spirituelles (psychologiques ou esthétiques) : trilogie d'éléments-clés dans laquelle entrée et salons de réception se trouvent mis en rapport étroit par le biais du péristyle, caractérisé moins par son jardin que par ses aménagements décoratifs en rapport avec l'eau courante; caractères publics marqués, luxe architectural et décoratif affiché et croissant, alliant conservatisme et innovations et recourant, non à des formes spécifiquement domestiques mais plus généralement à celles de l'architecture publique (religieuse, civile, voire funéraire) ; vaste extension au sol et recherche d'une position topographique stratégique (liée moins aux pratiques clientélaires qu'ostentatoires). Si dimension et luxe constituent globalement des critères de différenciation hiérarchique importants entre ces maisons, ils doivent toutefois être pondérés, en fonction du contexte local, par

111. Les dernières transformations de ces maisons à l'époque julioclaudienne qui consacrent le retrait d'une partie de sa fonction électorale à la domus, d'abord par l'interdiction des banquets à but électoral par Auguste, puis par le principe de cooptation au suffrage populaire sur recommandation impériale à la place de l'élection au suffrage populaire sur des bases clientélaires (Guilhembet, 2001, p. 222-227), ont pu trouver un écho dans les modèles provinciaux. 
la notion de grandeur ou de luxe subjectifs. Interviennent en outre dans cette appréciation des éléments de confort (balnéaires, pièces chauffées par hypocauste, vitrage...), plutôt associés à l'otium. Tous ces éléments quantitatifs et qualitatifs introduisent un écart incommensurable entre cet habitat rare et celui du commun qui restent malgré tout intimement liés par une promiscuité topographique nécessaire à l'ostentation du premier. Cette situation est l'exacte transposition architecturale des différences existant entre le sommet et la base de la pyramide sociale.

Même si les propriétaires restent généralement anonymes, l'étude des maisons sous un angle social permet de mieux cerner leur nature: il s'agit des membres des familles les plus puissantes des élites locales, engagées dans la course aux honneurs municipaux, en fait les personnes les plus proches du pouvoir impérial à l'échelon local et dont la domus, par sa situation au plus près du cœur civique et par ses formes empruntées à l'architecture publique, matérialise concrètement ce lien institutionnel. Les maisons à péristyle traduisent l'identité, quelquefois la personnalité qu'ils ont cherché à exprimer à travers elles selon leur rang, leurs moyens financiers et intellectuels. À ce titre, leurs maisons paraissent être un bon indice du haut degré d'intégration des élites municipales à un système, la marque d'une conscience aiguë de leur appartenance à une même communauté.

Sur un autre plan, on constate que les grandes phases de développement et de mutations de la maison à péristyle correspondent assez bien à celles de l'histoire politique et administrative de la Gaule. En dotant les primores civitatis d'un instrument leur permettant d'assumer efficacement et avec dignité leurs fonctions sociales, les domus à péristyle participent également du programme augustéen de contribution de l'architecture à la majesté de l'Empire et d'affirmation de la primauté de la civitas. Le décalage entre les diverses phases de conquête et l'apparition des maisons à péristyle peut s'expliquer par les lenteurs du démarrage de l'urbanisme en général, souvent peut-être pour des raisons plus juridiques ou techniques (création de la parure monumentale du forum, installation du réseau hydraulique) que financières ou économiques, éventuellement aussi par des préoccupations politiques (nécessité de pacification et consolidation jusqu'à l'époque augustéenne). Parallèlement à la consolidation de la Pax Romana et à la définitive intégration de la Gaule à l'Empire, ces maisons manifestent, surtout à partir de l'époque hadrianique, un accroissement constant de leur luxe, sans doute légitimé, comme dans la Rome augustéenne (Guilhembet, 2001, p. 222), par l'essor incontestable de la puissance de la cité. Le modèle de la maison à péristyle provinciale qui s'élabore au cours du Haut-Empire semble être rapidement passé du statut de simple mode d'expression de la réussite sociale à travers la réception passive du modèle du conquérant à celui d'acteur du dynamisme urbain. D'un bout à l'autre de son histoire, la maison livre cependant l'image, bien connue par ailleurs, d'une Gaule très conformiste et très "sage ", dont témoignent l'usage et le souci de conservation des décors anciens, les recréations archaïsantes (manifestations d'un ancrage dans une romanité bien antérieure à celle de la conquête ?), qui sont l'expression d'une caste plus que d'individus. Même quand commencent à apparaître des éléments plus personnels (scènes commémoratives, par exemple), les domus n'expriment que manifestations de loyalisme ou de conformisme, d'attachement aux valeurs romaines (phénomène qui se poursuit au Bas-Empire en contexte rural). Il semble que, dès l'époque claudionéronienne, mais surtout à partir de la fin du $\mathrm{I}^{\mathrm{er}} \mathrm{s}$., on puisse voir dans les caractères sans cesse plus marqués de l'ostentation typique de ces maisons, non une simple surenchère due à l'usure d'un modèle mais bien plutôt une influence du phénomène de renouvellement des aristocraties d'époque julio-claudienne, avec l'arrivée d'homines novi qui pourraient, comme on le constate dans d'autres domaines - funéraire notamment (Gros, 2001, p. 410) -, avoir introduit quelques éléments d'une nouvelle sensibilité en n'hésitant pas à afficher des valeurs autrefois condamnées et à affirmer sans cesse un peu plus l'image individuelle du propriétaire. Le lien de la maison à péristyle avec le pouvoir et avec le cadre urbain dans lequel il s'exerce sous la Pax Romana ressort également bien de leur déclin et de leur disparition subséquente à partir de la première moitié du III ${ }^{\mathrm{e}}$ s., à un moment d'importantes transformations politiques et de l'abandon des chefs-lieux de civitas comme siège exclusif du pouvoir, marquant ainsi la fin de ce qui a sans doute été l'un des instruments le plus réussi de l'œuvre unificatrice de Rome dans les provinces.

Pour conclure, on peut donc dire que, plus que l'habitat modeste (souvent peu expressif) et même plus que les monuments publics (collectifs et relativement formalistes), les domus à péristyle, conçues comme un modèle pour la société, se révèlent ainsi comme un miroir de celle-ci et les données chronologiques et matérielles les concernant (date, nombre, dimension, qualité de la réalisation, décor et luxe...) constituent donc un excellent baromètre de la santé économique et civique d'une civitas, et, plus largement, de l'état de romanisation de la société urbaine provinciale, à travers ses représentants les plus éminents. 


\title{
BIBLIOGRAPHIE
}

AE
APRAV
BAR
CAG
CIL
FIRA
ILA
ILS
JRA
RAN
SHA
SRA

\section{APULÉE}

L'Âne d'or ou les Métamorphoses, texte traduit et annoté par P. Grimal, Paris, Gallimard, 1975, $308 \mathrm{p}$.

\section{Cetius faventinus}

Abrégé d'architecture privée, texte établi, traduit et commenté par M. T. Cam, Paris, Les Belles Lettres, 2001, 175 p.

\section{CODE JUSTINIEN}

Institutes, texte édité par P. Krüger, Berlin, 1877 (rééd. 1963).

\section{Allag C.}

1993 : «La peinture à l'époque sévérienne : signification du décor d'une domus à Chartres ", in MOORMANN E. M. (DIR.), Functional and Spatial Analysis of Wall Painting, Proceedings of the $5^{\text {th }}$ International Congress on Ancient Wall Painting, Amsterdam, 8-12 sept. 1992, Leyde, Bulletin Antieke Beschaving (coll. Suppl. BABesch, Annual Papers on Classical Archaeology, 3), p. 111-114 et p. 271.

\section{ARNAUD P.}

1999: «Un flamine provincial des Alpes Maritimes à Embrun: flaminat provincial, incolatus et frontière des Alpes Maritimes », RAN, 32, p. 39-48.

\section{Balmelle C.}

1996 : "La maison romaine en Aquitaine ", in COLLeCtif, La Maison urbaine d'époque

\section{ABRÉVIATIONS}

\author{
L'Année épigraphique. \\ Association pour la promotion de la recherche en Vaucluse. \\ British Archaeological Report. \\ Carte archéologique de la Gaule. \\ Corpus Inscriptionum Latinarum. \\ Fontes Juris Romani Antiqui. \\ Inscriptions Latines d'Aquitaine. \\ Inscriptiones Latinae Selectae. \\ Journal of Roman Archaeology. \\ Revue archéologique de Narbonnaise. \\ Scriptores Historiae Augustae. \\ Service régional de l'archéologie.
}

\section{SOURCES ANTIQUES}

\section{DIGESTE}

Corpus Juris Civili, texte édité par T. Mommsen et P. Krüger, Berlin, 1920.

\section{PANÉGYRIQUES LATINS}

Panégyriques latins, tome I, livres I-V, texte établi et traduit par E. Galletier, Paris, Les Belles Lettres, 1949, LXXII et140 p.

\section{Pline Le JeUne}

Pline le Jeune, Lettres, tome II, livres IV-VI, texte établi et traduit par A.-M. Guillemin, Paris, Les Belles Lettres, 1927, 149 p.

\section{BIBLIOGRAPHIE}

romaine en Gaule Narbonnaise et dans les provinces voisines, Actes du colloque d'Avignon, 11-13 nov. 1994, Avignon, APRAV (coll. Documents d'archéologie vauclusienne, 6), p. 117-127.

2001 : Les Demeures aristocratiques d'Aquitaine : société et culture de l'Antiquité tardive dans le sud-ouest de la Gaule, Bordeaux, Pessac, Ausonius (coll. Suppl. à Aquitania, 10), 497 p.

\section{BARBET A.}

1980: «Le quatrième style de Pompéi », in Peintures murales en Gaule, Actes des séminaires, 1979, Dijon, Centre de recherches sur les techniques gréco-romaines, p. 65-76.

1983 : «La diffusion du III ${ }^{\mathrm{e}}$ style pompéien en Gaule », Gallia, 41, p. 111-165.

1987 : « La diffusion des I $^{\mathrm{er}}$, $\mathrm{II}^{\mathrm{e}}$ et $\mathrm{III}^{\mathrm{e}}$ styles pompéiens en Gaule », in COLlECTIF,

\section{PTOLÉmÉE}

Claudii Ptolemaei Geographia, texte établi, traduit et annoté en latin par K. Müller, Paris, FirminDidot, 2 vol., 1883-1901, 1023 p.

\section{SCRIPTORES HISTORIAE AUGUSTAE}

Histoire Auguste. Les empereurs romains des II et $I I I^{e}$ siècles, texte établi et traduitparA. Chastagnol, édition bilingue latin-français, Paris, Robert Laffont (coll. Bouquins), 1994, 1244 p.

\section{VITRUVE}

De Architectura, livre VI, texte établi, traduit et commenté par L. Callebat, Paris, Les Belles Lettres, 2004, 254 p.

Pictores per provincias, Actes du $3^{e}$ colloque international sur la peinture murale romaine, Avenches, 1986, Avenches (coll. Cahiers d'archéologie romande, 43), p. 7-27 et p. 251.

1990: «L'emploi des couleurs dans la peinture murale romaine antique: marqueurs "chronologiques" et révélateurs du "standing" social ? ", in Pigments et colorants de l'Antiquité et du Moyen Âge, Actes du colloque international du CNRS, Orléans, 1988, Paris, éd. du CNRS, p. 255-271.

Barbet A., Allag C., BecQ G., GROETEMBRIL S., LEFÈVRE J.-F., MONIER F.

1996 : «Le décor peint dans la maison », in Collectif, La Maison urbaine d'époque romaine en Gaule Narbonnaise et dans les provinces voisines, Actes du colloque 
d'Avignon, 11-13 nov. 1994, Avignon, APRAV (coll. Documents d'archéologie vauclusienne, 6), p. 25-42.

\section{Barbet A., Girardy-Caillat C., BOST J.-P.}

2003 : «Peintures de Périgueux : édifice de la rue des Bouquets ou la domus de Vésone -I- Les peintures en place ", Aquitania, 19, p. 81-126.

\section{BARbeT A., MONIER F., BOST J.-P.,} STERNBERG M.

2004: «Peintures de Périgueux : édifice de la rue des Bouquets ou la domus de Vésone -II- Les peintures fragmentaires ", Aquitania, 20, p. 149-219.

Barbet A., Monier F., LOUStaud J.-P.

1993 : «Les peintures murales de la Maison des Nones de Mars à Limoges ", Aquitania, 11 , p. 63-111.

\section{BARrière C., SARRAdet M.}

1996: Domus "Pompéia": fouilles de la rue des Bouquets à Périgueux (Dordogne), Chancelade, ADRAHP (coll. Suppl. Documents d'archéologie et d'histoire périgourdines, 2), 164 p., 8 pl.

\section{BAtTelli P.}

1998 : «L'ingresso della domus come confine tra città e abitazione ", Studi romani, XLVI, 3-4, p. 281-301.

BENOÎT F.

1947: «La maison à double péristyle du Jardin de Grassi à Aix-en-Provence ", Gallia, V, 1 p. 98-122.

\section{BINET E.}

1996 : «Le site antique du "Palais des Sports" à Amiens ", Revue du Nord, LXXVIII, 318, p. 83-96.

BLAGG T. F. C.

1984: «An examination of the connexions between military and civilian architecture in Roman Britain ", in BLAGG T. F. C., KING A. C. (DIR.), Military and Civilian in Roman Britain: Cultural Relationships in Frontier Province, Oxford, Archaeopress (coll. BAR, British Series, 136), p. 249-263.

\section{BLANCHARD-LEMÉE M., OLIVIER A.,} REBOURG A.

1986 : «Deux maisons à pavements d'Augustodunum, Autun (Saône-et-Loire) ", Gallia, 44, 1, p. 121-149.

BÖGLI H. ET AL.

1970-1971 : «Insula 16 Est : rapport sur les fouilles exécutées en 1965-1966 ", Bulletin de l'Association Pro Aventico, 21, p. 19-39 et pl. 17-33.

BOST J.-P.

1993: «Exploits amoureux à Limoges au III $^{\mathrm{e}} \mathrm{s}$. après J.-C. : trois graffiti de la "Maison des Nones de Mars" ", Travaux d'archéologie limousine, 13, p. 53-57.

\section{BOUET A.}

1996: «Les thermes des maisons urbaines en Gaule Narbonnaise ", in COLLECTIF, La Maison urbaine d'époque romaine en Gaule Narbonnaise et dans les provinces voisines, Actes du colloque d'Avignon, 11 13 nov. 1994, Avignon, APRAV (coll. Documents d'archéologie vauclusienne, 6), p. 169-183.

2001 : «Les collèges dans la ville antique : le cas des Subaediani ", Revue archéologique, nouvelle série, 2001, 2, p. 227-278.

\section{BROISE P.}

1969 : «Éléments d'un ordre toscan provincial en Haute-Savoie ", Gallia, XXVII, 1, p. 15-22.

\section{BRULET R.}

1996: «La maison urbaine en Gaule Belgique et en Germanie inférieure », in COLlectif, La Maison urbaine d'époque romaine en Gaule Narbonnaise et dans les provinces voisines, Actes du colloque d'Avignon, 11-13 nov. 1994, Avignon, APRAV (coll. Documents d'archéologie vauclusienne, 6), p. 72-97.

\section{Callebat L.}

1999 : «Observations sur le vocabulaire de l'habitat romain ", in PETERSMANN H., KETTEMANN R. (DIR.), Latin vulgairelatin tardif, Actes $d u V^{e}$ colloque international sur le latin vulgaire et tardif, Heidelberg, 5-8 sept. 1997, Heidelberg, Carl Winter, p. 519-527.

CAM M. T.

2001: Cetius Faventinus : abrégé d'architecture privée, Paris, Les Belles Lettres, 175 p.

\section{CébeillaG-Gervasoni M., LAMOINe L.,} TRÉMENT F. (DIR.)

2004: Autocélébration des élites locales dans le monde romain : contextes, images, textes (II $s$. av. J.-C./III s. apr.J.-C.), Actes du colloque de Clermont-Ferrand, 21-23 nov. 2003, Clermont-Ferrand, Presses universitaires Blaise-Pascal (coll. Erga, 7), 570 p.

Cerruti-DÉrofF M.-C. (DIR.)

1996: Annuaire des opérations de terrain en milieu urbain 1996, Tours, Ministère de la Culture, Direction du Patrimoine, 136 p.
Chardron-Picault P., Lorenz J., RAT P., SAURON G. (DIR.)

2004 : Les Roches décoratives dans l'architecture antique et du haut Moyen Âge, Table ronde du Centre d'archéologie et du patrimoine, Autun, 18-19 nov. 1999, Paris, Comité des travaux historiques et scientifiques (coll. Archéologie et histoire de l'art, 16), 389 p.

\section{Chastagnol A.}

1994 : Histoire Auguste. Les empereurs romains des $I I^{e}$ et $I I I^{e}$ siècles, édition bilingue latinfrançais Paris, Robert Laffont (coll. Bouquins), $1244 \mathrm{p}$.

\section{CLARKE J. R.}

1991 : The Houses of Roman Italy, 100 B.C.A.D. 250: Ritual, Space and Decoration, Berkeley, Oxford, University of California Press, XXVI p. et 411 p., 3 cartes, 24 pl.

\section{CoArelli F}

1989 : «La casa dell'aristocrazia romana secondo Vitruvio ", in GEERTMAN H., JONG J. J. DE (DIR.), Munus non ingratum, Proceedings of the international symposium on Vitruvius' De Architectura and the Hellenistic and Republican Architecture, Leiden, 20-23 janv. 1987, Leyde, Bulletin Antieke Beschaving (coll. Suppl. BABesch, Annual Papers on Classical Archaeology, 2), p. 178-187.

\section{ColleGtiF}

1987 : Pictores per provincias, Actes du $3^{e}$ colloque international sur la peinture murale romaine, Avenches, 1986, Avenches (coll. Cahiers d'archéologie romande, 43), $263 \mathrm{p}$.

1991: La Casa urbana hispanoromana, Actes du colloque de Saragosse, 16-18 nov. 1988, Saragosse, Institución Fernando el Católico, 386 p.

1994: Maîtres et élèves, célébrités et savants : l'École normale supérieure, 1794-1994, Paris, Archives nationales, $287 \mathrm{p}$.

1996a: La Maison urbaine d'époque romaine en Gaule Narbonnaise et dans les provinces voisines, Actes du colloque d'Avignon, 1113 nov. 1994, Avignon, APRAV (coll. Documents d'archéologie vauclusienne, 6), $261 \mathrm{p}$.

1996b: La Maison urbaine d'époque romaine. Atlas des maisons de Gaule Narbonnaise, Avignon, APRAV (coll. Documents d'archéologie vauclusienne, 6), 420 p.

1997: Bilan scientifique de la région Bretagne 1997, Paris, Ministère de la Culture et de la Communication, 117 p.

\section{CotTiCa D.}

1994 : « La casa urbana in Britannia e la sua 
evoluzione: una breve anali complessiva ", Rivista di archeologia, 18, p. 90-104.

\section{COUPRY J.}

1975 : «Informations archéologiques de la circonscription d'Aquitaine ", Gallia, 33, 2, p. 461-490.

DE AlbENTIIS E.

1990 : La Casa dei Romani, Milan, Longanesi, $350 \mathrm{p}$.

\section{Desbat A.}

1984 : Les Fouilles de la rue des Farges à Lyon: 1974-1980, Lyon, Groupe lyonnais de recherche en archéologie gallo-romaine, $107 \mathrm{p}$.

1991 (2ééd.) : «La maison gallo-romaine ", in Goudineau C., Guilaine J. (DIR.), De Lascaux au Grand Louvre: archéologie et histoire en France, Paris, éd. Errance, p. 296-299.

\section{Desbat A., Leblanc O., Prisset J.-L.}

1994: La Maison des Dieux Océan à SaintRomain-en-Gal (Rhône), Paris, CNRS Éditions (coll. Suppl. à Gallia, 55), 276 p., 175 fig., 125 photos.

\section{Desbat A., MANDY B.}

1991: «Le développement de Lyon à l'époque augustéenne: l'apport des fouilles récentes ", in GOUDINEAU C., REBOURG A. (DIR.), Les Villes augustéennes de Gaule, Actes du colloque international d'Autun, 6-8juin 1985, Autun, Société éduenne des lettres, sciences et arts, p. 79-97.

\section{Deschamps S., Guérin F., Pascal J.,}

PIRAULT L.

1992 : «Ratiatum (Rezé, Loire-Atlantique) : origine et développement de l'organisation urbaine ", Revue archéologique de l'Ouest, 9, p. 111-127.

\section{DESSALES H.}

2003 : "Piscina et salientibus additis: fontaines et jeux d'eau dans la maison romaine ", Vita Latina, 169, p. 2-10.

2004: «Les fontaines privées de la Gaule romaine ", Dossiers d'archéologie, 295, p. $20-29$.

\section{DRERUP H.}

1959: "Bildraum und Realraum im römischen Architektur », Mitteilungen des deutschen archäologischen Instituts. Römische Abteilung, LXVI, p. 147-174.

\section{DRINKWATER J. F.}

1978 : «The rise and fall of the Gallic Iulii: aspects of the development ot the aristocraty of the Three Gauls under the Early Empire », Latomus, XXXVII, p. 817-850.

1979 : «Gallic personnal wealth », Chiron, 9, p. $237-242$.

\section{DUMONT J. C.}

1990 : «Le décor de Trimalcion », Mélanges d'archéologie et d'histoire de l'École française de Rome, 102, 2, p. 959-981.

\section{DUNCAN-JONES R. P.}

1965: «An epigraphic survey of costs in Roman Italy », Papers of the British School at Rome, XXXIII, n. s. XX, p. 189-306.

\section{DWYER E.}

1994 ( $2^{\mathrm{e}}$ éd.) : "The pompeian atrium house in theory and practice ", in GAZDA E. K. (DIR.), Roman Art in the Private Sphere : New Perspectives on the Architecture and Decor of the Domus, Villa and Insula, Ann Arbor, University of Michigan Press, p. 25-48.

ELLIS S. P.

1988: "The end of the Roman house ", American Journal of Archaeology, 92, 4, p. 565-576.

2000 : Roman Housing, Londres, Duckworth, 224 p.

\section{ERISTOV H.}

1987 : «Les peintures murales provinciales d'époque flavienne ", in COLlECTIF, Pictores per provincias, Actes du $3^{e}$ colloque international sur la peinture murale romaine, Avenches, 1986, Avenches (coll. Cahiers d'archéologie romande, 43), p. 45-55.

1994: «Un témoignage de loyalisme impérial dans un décor peint? ", Gallia, 51, p. 217-232.

\section{ERISTOV H., VAUGIRAUD S. DE}

1994 : «Peintures murales gallo-romaines du 12 rue de l'Abbé-de-l'Épée ", Cahiers de la Rotonde, 15, p. 65-158.

\section{ÉTIENNE R.}

1960 : Le Quartier nord-est de Volubilis, Paris, De Boccard, 2 vol., 190 p. et XVIII pl.

\section{FREMERSDORF F.}

1956: Das römische Haus mit dem DionysosMosaik vor dem Südportal des Kölner Domes, Berlin, Rheinischen Landesmuseums (coll. Bonner Jahrbücher, 33), 136 p., 42 pl. et 1 plan.

\section{FRIZOT M.}

1977 : Stucs de Gaule et des provinces romaines : motifs et techniques, Dijon, Université de Dijon (coll. Publication du Centre de recherches sur les techniques grécoromaines, 7), $258 \mathrm{p}$.

\section{FUCHS M}

1987 : «La peinture murale sous les Sévères », in COLlectif, Pictores per provincias, Actes $d u 3^{e}$ colloque international sur la peinture murale romaine, Avenches, 1986, Avenches (coll. Cahiers d'archéologie romande, 43), p. 67-77 et pl. XII p. 262.

\section{GARNSEY P.}

1976: "Demolition of houses and the law », in FINLEYM. I. (DIR.), Studies in Roman Property, Cambridge, Cambridge University Press, p. 133-136.

\section{Girardy-GAillat C.}

1992 : «Périgueux-Vesunna», in COLLECTIF, Villes et agglomération urbaines antiques $d u$ sud-ouest de la Gaule, Actes du $2^{e}$ colloque Aquitania, Bordeaux, 13-15 sept. 1990, Bordeaux, Association Aquitania (coll. Suppl. à Aquitania, 6), p. 125-129.

\section{GONZÁLEZ J.}

1986: "The lex Irnitana: a new copy of the Flavian municipal law », Journal of Roman Studies, LXXVI, p. 147-243 et pl. V-XXIII.

\section{GoudineaU C.}

1979 : Les Fouilles de la Maison au Dauphin: recherches sur la romanisation de Vaisonla-Romaine, Paris, CNRS Éditions (coll. Suppl. à Gallia, XXXVII), 2 vol., 325 p., $101 \mathrm{pl}$. et XIV dépliants.

1993 : «Bibracte : les habitants et leurs habitations ", in GOUdineau C., PEYRE C. (DIR.), Bibracte et les Éduens : à la découverte du peuple gaulois, Paris, éd. Errance, p. 54-74.

GoudineaU C., KISCH Y. DE

1991 : Vaison-la-Romaine, Paris, éd. Errance, $160 \mathrm{p}$.

GoudineaU C., Rebourg A. (DIR.)

1991: Les Villes augustéennes de Gaule, Actes $d u$ colloque international d'Autun, 6-8 juin 1985, Autun, Société éduenne des lettres, sciences et arts, 152 p.

\section{Grimal P., GUEy J.}

1937 : « À propos des "bains de Livie" au Palatin ", Mélanges d'archéologie et d'histoire, LIV, 1-4, p. 142-164.

\section{GROS P.}

1997: «Maisons ou sièges de corporation? Les traces archéologiques du phénomène associatif dans la Gaule méridionale », Comptes rendus des séances de l'Académie 
des inscriptions et belles-lettres, janvier-mars, p. 213-241.

2001 : L'Architecture romaine du début du III siècle av.J.-C. à la fin du Haut-Empire -2Maisons, palais, villas et tombeaux, Paris, Picard, 527 p.

2004: «La basilique dans la maison des notables ", in CÉBEILLAC-GERVASONI M., LAMOINE L., TRÉMENT F. (DIR.), Autocélébration des élites locales dans le monde romain : contextes, images, textes (II $s$. av. J.-C./III s. apr. J.-C.), Actes du colloque de Clermont-Ferrand, 21-23 nov. 2003, Clermont-Ferrand, Presses universitaires Blaise-Pascal (coll. Erga, 7), p. 311-328.

\section{GUIDOBALDI F.}

1986 : «L'edilizia abitativa unifamiliare nella Roma tardoantica », in GIARDINA A. (DIR.), Società romana e impero tardoantico-II- Roma : politica, economia, paesaggio urbano, Rome, Bari, Laterza, p. 165-237.

\section{GUILHEMBET J.-P.}

2001: «Les résidences aristocratiques de Rome, du milieu du $I^{\mathrm{er}}$ siècle avant n. è. à la fin des Antonins ", Pallas, 55, p. 215-241.

Haldimann M.-A., Ramjoué E., Simon C. 1991 : «Les fouilles de la cour de l'ancienne prison Saint-Antoine : une vision renouvelée de la Genève antique ", Archéologie suisse, 14, 2, p. 194-204.

HODGE A. T.

1992: Roman Aqueducts and Water Supply, Londres, Duckworth, 504 p.

JACOB J.-P., D'ANNA A. ET $A L$.

1990 : «Provence-Alpes-Côte d'Azur », Gallia Informations, 1990, 1-2, p. 131-234.

\section{JACQUES F.}

1984 : Le Privilège de liberté : politique impériale et autonomie municipale dans les cités de l'Occident romain (161-244), Rome, École française de Rome (coll. de l'École française de Rome, 76), 867 p.

\section{JACQUES F., SCHEID J.}

1990: Rome et l'intégration de l'Empire: 44 av. J.-C./260 apr. J.-C. -I- Les Structures de l'Empire romain, Paris, PUF, 412 p.

\section{JOCKEY P.}

1996 : « Le décor sculpté de la maison urbaine en Gaule Narbonnaise ", in COLlECTIF, La Maison urbaine d'époque romaine en Gaule Narbonnaise et dans les provinces voisines, Actes du colloque d'Avignon, 1113 nov. 1994, Avignon, APRAV (coll.
Documents d'archéologie vauclusienne, 6), p. 195-211

\section{KOLENDO J}

1991: «L'aristocratie municipale dans les provinces rhénanes et danubiennes à l'époque du Haut-Empire ", JRA, 4 p. $327-330$.

Koloski-OStrow A. O., DE HAAN N., DE KLEIJN G., PIRAS S.

1997: «Water in the Roman town: new research from Cura Aquarum and the Frontinus Society », JRA, 10, p. 182-191.

\section{LAFON X.}

1995 : «Dehors ou dedans?: le vestibulum dans les domus aristocratiques à la fin de la République et au début de l'Empire », Klio, 77, p. 405-423.

\section{LANCHA J.}

1997 : Mosaïque et culture dans l'Occident romain : $I^{e r}-I V^{e} s .$, Rome, l'Erma di Bretschneider, 439 p., 126 pl.

Lapart J., Petit C.

1993 : Le Gers, Paris, Académie des inscriptions et belles-lettres et Ministère de la Culture et de la Communication (coll. $C A G, 32), 354$ p.

\section{LASFARGUES J.}

1982 : «Informations de la circonscription de Rhône-Alpes », Gallia, 40, 2, p. 389-428.

\section{LAVAGNE $\mathbf{H}$}

1994 : «Le pavement de la rue des Magnans à Aix-en-Provence et la naissance des trames à "décor multiple" dans la mosaïque gallo-romaine », Gallia, 51, p. 202-215

1996 : « La mosaïque et la maison : quelques textes sur les usages de la mosaïque ", in COLleCtif, La Maison urbaine d'époque romaine en Gaule Narbonnaise et dans les provinces voisines, Actes du colloque d'Avignon, 11-13 nov. 1994, Avignon, APRAV (coll. Documents d'archéologie vauclusienne, 6), p. 43-47.

\section{LE MASNE DE CLERMONT N.}

1987: «Les fouilles de l'ancien évêché de Poitiers (Vienne)», Aquitania, V, p. 149-175.

\section{LEVEAU $P$}

1996: «L'eau dans la maison à l'époque romaine », inCOLLECTIF, La Maisonurbaine d'époque romaine en Gaule Narbonnaise et dans les provinces voisines, Actes du colloque d'Avignon, 11-13 nov. 1994, Avignon,
APRAV (coll. Documents d'archéologie vauclusienne, 6), p. 155-167.

LEVEAU P. (DIR.)

1988 (1 ${ }^{\text {re }}$ éd. : 1985): L'Origine des richesses dépensées dans l'Antiquité, Actes du colloque organisé par l'UER d'histoire de l'université d'Aix-en-Provence, 11-12 mai 1984, Aix-enProvence, Publications de l'Université de Provence, $286 \mathrm{p}$

LOREnZ J., TARdY D., COUlON G. (DIR.) 2000 : La Pierre dans la ville antique et médiévale, Actes du colloque d'Argentomagus, 30-31 mars 1998, Saint-Marcel, Musée d'Argentomagus et Tours, FERAC (coll. Mémoire du musée d'Argentomagus, 3 et Suppl. à la Revue archéologique du Centre de la France, 18), 186 p.

\section{LOUSTAUD J.-P.}

1987: «Une riche demeure gallo-romaine près du forum d'Augustoritum ", Travaux d'archéologie limousine, 8, p. 17-36.

1992 : «Les fouilles du jardin "des Récollets de Sainte-Valérie" à Limoges (2 ${ }^{\mathrm{e}}$ partie) : la Maison des Nones de Mars ", Travaux d'archéologie limousine, 12, p. 23-111.

1996: «Les résidences sur terrasses à Augustoritum, signes de romanisation ostentatoire ", Travaux d'archéologie limousine, 16, p. 39-54.

2000 : Limoges antique, Limoges, Association des Antiquités historiques du Limousin (coll. Suppl. aux Travaux d'archéologie limousine, 5), 387 p.

MCKAY A. G.

1975 : Houses, Villas and Palaces in the Roman World, Londres, Thames and Hudson, 288 p.

MARCAdé J., SAbrié M., SAbrié R.

1999 : «Sculptures du Clos de la Lombarde à Narbonne (Aude) », RAN, 32, p. 309-334.

MAURIN L. (DIR.)

1988: Les Fouilles de "Ma Maison": études sur Saintes antique, Bordeaux, Association Aquitania (coll. Suppl. à Aquitania, 3), 302 p.

MAY R.

1986 : Saint-Bertrand-de-Comminges (Lugdunum Convenarum) : le point sur les connaissances, Toulouse, Association pour la promotion du patrimoine archéologique et historique en Midi-Pyrénées, 187 p.

\section{MAZERAN R}

1996 : «Les marbres de la maison romaine de l'Aire du Chapitre à Aix-en-Provence », 
in Collectif, La Maison urbaine d'époque romaine en Gaule Narbonnaise et dans les provinces voisines, Actes du colloque d'Avignon, 11-13 nov. 1994, Avignon, APRAV (coll. Documents d'archéologie vauclusienne, 6), p. 213-217.

\section{MEYER K. E.}

1999 : «Axial peristyle houses in the western Empire ", JRA, 12, p. 101-121.

\section{MOORMANN E. M. (DIR.)}

1993: Functional and Spatial Analysis of Wall Painting, Proceedings of the $5^{\text {th }}$ International Congress on Ancient Wall Painting, Amsterdam, 8-12 sept. 1992, Leyde, Bulletin Antieke Beschaving (BABesch) (coll Suppl. BABesch, Annual Papers on Classical Archaeology, 3), 276 p.

\section{NAVEAU J}

1992: La Mayenne, Paris, Académie des inscriptions et belles-lettres et Ministère de la Culture et de la Communication (coll. CAG, 53), 176 p.

\section{Paillet J.-L., Petit C.}

1992 : «Nouvelles données sur l'urbanisme de Lugdunum des Convènes: prospection aérienne et topographie urbaine ", Aquitania, X, p. 109-144.

\section{PaUnier D.}

1996 : «L'état de la question en Suisse », in COllectif, La Maison urbaine d'époque romaine en Gaule Narbonnaise et dans les provinces voisines, Actes du colloque d'Avignon, 11-13 nov. 1994, Avignon, APRAV (coll. Documents d'archéologie vauclusienne, 6), p. 99-115.

\section{Paunier D., Desbat A., Meylan F.}

2002: «Les premiers habitats romanisés en Gaule du Centre-Est: un témoignage de l'aristocratie indigène? ", in GUICHARD V., PERRIN F. (DIR.), L'Aristocratie celte à la fin de l'âge du Fer (II s. av.J.-C./Irer apr. J.-C.), Actes de la table ronde de Glux-en-Glenne, 10-11 juin 1999, Glux-en-Glenne, Centre archéologique du Mont Beuvray (coll. Bibracte, 5), p. 271-287.

PAUnier D., LUGINBÜHL T. (DIR.)

2004 : Bibracte : le site de la maison 1 du Parc aux Chevaux (PC 1) des origines de l'oppidum au règne de Tibère, Glux-en-Glenne, Centre archéologique du Mont-Beuvray (coll. Bibracte, 8), 468 p., 7 pl. couleur h. t.

\section{Pelletier A.}

2000: «Les inscriptions viennoises des jumeaux Coelii et le recrutement des décurions en Gaule », RAN, 33, p. 2-7.

\section{PERRIN Y.}

1997: «Peinture et architecture : statut du décor, statut de l'édifice, statut de la recherche », JRA, 10, p. 355-362.

\section{PESANDO F.}

1997 : Domus, edilizia privata e società pompeiana fra III e I secolo a. C., Rome, l'Erma di Bretschneider, $393 \mathrm{p}$.

PICARD G.-C.

1987-1988: «L'enrichissement par le commerce dans la Gaule romaine à l'époque impériale », Bulletin archéologique du Comité des travaux historiques et scientifiques, 23-24, p. 7-37.

\section{PINON P.}

1983 : «L'“invention” de la maison romaine par les architectes de l'époque classique ", in Chevallier R. (DIR.), Présence de l'architecture et de l'urbanisme romains, Actes du colloque Caesarodunum, XVIIIbis, Paris, Les Belles Lettres, p. 281-305.

\section{Plassot E.}

1995 : «Le quartier Saint-Pierre : la maison aux Xénia », in DELAVAl E., BELlon C., CHASTEL J. ET AL. (DIR.), Vaise, un quartier de Lyon antique, Lyon, SRA Rhônes-Alpes (coll. Documents d'archéologie en Rhône-Alpes, 11), p. 71-130.

\section{Plassot E., Desbat A.}

2003: "Le site de la rue du Souvenir", in POUX M., SAVAY-GuERraz H. (DIR.), Lyon avant Lugdunum, Lyon, Musée de la civilisation gallo-romaine, p. 130-133.

PRISSET J.-L., BRISSAUd L., LEblanc O.

1994: «Évolution urbaine à Saint-Romainen-Gal: la rue du Commerce et la maison aux Cinq Mosaïques ", Gallia, 51, p. 1-133.

\section{RIGGSBY A. M.}

1997: "Public" and "private" in Roman culture: the case of cubiculum ", JRA, 10 , p. $36-56$

\section{RODRÍGEZ AlMEIDA E.}

1981 : Forma Vrbis Marmorea. Aggiornamento generale 1980, Rome, Quasar, 2 vol., XVIII p. et 172 p., LXI pl.

\section{ROULAND N.}

1979 : Pouvoir politique et dépendance personnelle dans l'Antiquité romaine: genèse et rôle des rapports de clientèle, Bruxelles, Latomus (coll. Latomus, 166), 658 p.
SABLAYROLLES R.

1988 : "La pompe romaine de Périgueux », Aquitania, VI, p. 141-156.

\section{SAbrié M., SAbrié R.}

1999 : «Les manifestations du culte impérial à travers la peinture », RAN, 32, p. 57-63.

SAbrié M., SAbrié R., Solier Y.

1987: La Maison à portiques du Clos de la Lombarde à Narbonne et sa décoration murale: fouilles 1975-1983, Paris, éd. du CNRS (coll. Suppl. à la RAN, 16), 370 p.

\section{SAliou C.}

1990 : «Maison et paysage urbain dans les villes du Proche-Orient $\left(\mathrm{II}^{\mathrm{e}}-\mathrm{V}^{\mathrm{e}}\right.$ siècles apr. J.-C.) ", Histoire de l'Art, 9/10, mai 1990, p. 9-20.

1994 : Les Lois du bâtiment : voisinage et habitat urbain dans l'Empire romain, recherches sur les rapports entre le droit et la construction privée du siècle d'Auguste au siècle de Justinien, Beyrouth, Institut français d'archéologie du Proche-Orient (coll. Bibliothèque archéologique et historique, CXVI), 340 p.

\section{TARDY D.}

1992 : «Le décor architectonique : l'exemple de Saintes sous le Haut-Empire ", in COLLECTIF, Villes et agglomération urbaines antiques du sud-ouest de la Gaule, $2^{e}$ colloque Aquitania, Bordeaux, 13-15 sept. 1990, Bordeaux, Fédération Aquitania (coll. Suppl. à Aquitania, 6), p. 323-334.

\section{THÉBERT Y.}

1985 : «Vie privée et architecture domestique en Afrique romaine ", in ARIÈs P., Duby G. (DIR.), Histoire de la vie privée de l'Empire romain à l'an mil, Paris, Seuil, p. 305-397.

1993 : «Private and public spaces : the components of the domus", in D'AMBRA E. (DIR.), Roman Art in Context : an Anthology, Englewood Cliffs, New Jersey, Londres, Prentice Hall, p. 213-237.

\section{VIPARD P.}

1991 : «Une statue récemment découverte à Vieux (Calvados) », Gallia, 47, p. 251-255. 1992: "Note sur une statue découverte à Vieux (Calvados)", in L'Art en BasseNormandie, Actes du XXVI congrès des sociétés historiques et archéologiques de Normandie, Caen, Conseil général du Calvados, Service des archives départementales, vol. I, p. 69-78.

1996: Une domus du quartier des thermes d'Aregenua (Vieux, Calvados): contribution à l'histoire de l'habitat urbain en Gaule 
romaine, thèse de Doctorat d'Histoire nouveau régime, Université Paris-IV, 4 vol., 899 p., 225 fig., 176 photos, 1 plan.

1998: La Maison du "Bas de Vieux": une riche habitation du quartier des thermes d'Aregenua (Vieux, Calvados), Caen, SRA Calvados, 125 p.

1999 : «Les matériaux architecturaux en terre cuite dans la domus du "Bas de Vieux" ", in SAN JUAN G., MANEUVRIER J. (DIR.), L'Exploitation ancienne des roches dans le Calvados: histoire et archéologie, Caen, Conseil général du Calvados, Service départemental d'archéologie, p. 208-219.

2001 : «Le rôle du décor dans les parties officielles d'une domus à péristyle du début du III $^{\mathrm{e}}$ s. : le cas de la Maison au Grand Péristyle (Vieux, Calvados)", Revue $d u$ Nord, 83, n 343, p. 21-33.

2001-2002 : «Un aménagement architectural méconnu : les portiques fenêtrés dans les domus du Haut-Empire ", in BEDON R. (DIR.), Amœenitas urbium : les agréments de la ville à l'époque romaine, Actes du colloque de Limoges, 26-27 mai 2000, Limoges, Presses universitaires de Limoges (coll. Caesarodunum, XXXV-XXXVI), p. 39-56.

2002: La Cité d'Aregenua (Vieux, Calvados), chef-lieu des Viducasses, Paris, éd. Exé Productions, $204 \mathrm{p}$.

2003 : «Les portiques fenêtrés dans les domus du Haut-Empire romain ", Bulletin archéologique du comité des travaux historiques et scientifiques, 30, p. 99-134.

2004: «Quelques manifestations d'autocélébration des membres des élites urbaines dans le cadre domestique », in CÉBEILlaC-GERVASONI M., LAMOINE L., TrÉmEnT F. (DIR.), Autocélébration des élites locales dans le monde romain : contextes, images, textes (II ${ }^{e}$ s. av. J.-C./III $I^{e}$ s. apr. J.-C.), Actes du colloque de Clermont-Ferrand, 2123 nov. 2003, Clermont-Ferrand, Presses universitaires Blaise-Pascal (coll. Erga, 7), p. 383-403.

À paraître : «Le verre à vitre dans l'architecture romaine du Haut-Empire ", in Verre et fenêtre, Colloque international de ParisLa Défense-Versailles, 13-15 oct. 2005.

\section{VIPARD P., RIOULT M.}

1999: «L'utilisation de la pierre dans la demeure gallo-romaine de Vieux ", in SAN JUAN G., MANEUVRIER J. (DIR.), L'Exploitation ancienne des roches dans le Calvados: histoire et archéologie, Caen, Conseil général du Calvados, Service départemental d'archéologie, p. 188-203.

\section{VIPARD P., SAVARY X.}

2004: «L'emploi du schiste bitumineux d'Autun à Aregenua (Vieux, Calvados) ", in CHARdron-PiCAUlt P., LOREnZ J., RAT P., SAURON G. (DIR.), Les Roches décoratives dans l'architecture antique et du haut Moyen Âge, Table ronde du Centre d'archéologie et du patrimoine, Autun, 18-19 nov. 1999, Paris, Comité des travaux historiques et scientifiques (coll. Archéologie et histoire de l'art, 16), p. 153-165.

\section{WACHER J.}

1981 (1 $1^{\text {re }}$ éd. : 1975) : The Towns of Roman Britain, Londres, Batsford, 460 p.

\section{WALLACE-HADRILL A.}

1988: "The social structure of the Roman house ", Papers of the British School at Rome, LVI, p. 43-97.
2001 : «Case e società ", in Guzzo P. G. (DIR.), Pompei. Scienza e società, $250^{\circ}$ anniversario degli scavi di Pompei, Convegno internazionale, Naples, 25-27 nov. 1998, Milan, Electa, p. 113-117.

\section{WALTER H.}

1970 : La Colonne ciselée en Gaule romaine, Paris, (coll. Annales littéraires de l'Université de Besançon, 119), 139 p., XXXIX pl.

\section{WILSON R. J. A.}

1996: «Tot aquarum tam multis necessariis molibus... Recent studies on aqueducts and water supply », JRA, 9, p. 5-29.

\section{WISEMAN T. P.}

1987: «Conspicui postes tectaque digna deo: the public image of aristocratic houses in the Late Republic and Early Empire ", in L'Urbs: espace urbain et histoire (I ${ }^{e r}$ siècle av. J.-C.-III siècle apr. J.-C.), Rome, École française de Rome (coll. de l'École française de Rome, 98), p. 393-413.

\section{ZACCARIA RUGGIU A.}

1990 : «L'intervento pubblico nella regolamentazione dello spazio privato. Problemi giuridici », Rivista di archeologia, XIV, p. 77-94.

1995 : Spazio privato e spazio pubblico nella città romana, Rome, École française de Rome (coll. de l'École française de Rome, 210), $607 \mathrm{p}$.

1996: «Maiestas urbis: il contributo dell'architettura privata all'immagine pubblica della città ", in BEDON R. (DIR.), Les Villes de la Gaule Lyonnaise, Limoges, Paris, éd. de l'École normale supérieure (coll. Caesarodunum, XXX), p. 415-454. 